\title{
Understanding the impacts of humanitarian Menstrual Health Management for women's confidence and agency: A case study on the Thai/Burma border
}

\section{Tessa Marie Bardsley}

A thesis submitted to Victoria University of Wellington in partial fulfilment of requirements for the degree of Master of Development Studies

School of Geography, Environment and Earth Sciences, Te Herenga Waka Victoria University of Wellington 2020 


\section{Abstract}

Menstrual Health Management (MHM) is a growing focus within global humanitarian and development work. MHM aims to increase women and girls' ability to care for their menstruating bodies through period products, hygiene facilities and education. It also seeks to challenge wider barriers that women face during menstruation - stigma, shame and 'period poverty' (the lack of access to menstrual products). NGOs promote 'menstrual activism' campaigns to 'Reduce the stigma' and 'Help women and girls. Period.' They tell stories of girls who, with their menstrual products, can now attend school during their period.

Academic studies into the complexities of such programmes are sparse, however; particularly studies which privilege the experiences of the women involved and affected. Research on menstruation within the humanitarian field is also limited. This is despite the fact that women living in refugee camps and precarious spaces can have heightened difficulties due to scarcity of menstrual resources, disruption of support networks and facilities with inadequate privacy. Contextually and culturally embedded research that recognises the contributions and perspectives of these women can strengthen humanitarian MHM programmes and scholarship so that menstruating women can feel confidence and agency rather than stigma and shame.

In light of these gaps, this study investigates Partners Relief \& Development's (Partners) 'Days for Girls' menstrual health programme in Thailand. The programme employs migrant women from Burma to make reusable menstrual hygiene kits and donates these kits to women in conflict-affected areas. The research worked within a feminist epistemology and mixed-methods methodology informed by principles of Appreciative Inquiry, to explore what is working well and what could be improved in Partners' menstrual health programme. It involved refugees and migrants from Myanmar living over the border in Thailand, as well as the programme's staff.

Through thematic analysis, I found that the Days for Girls programme improves women's agency (through increased community participation) and confidence (through menstrual literacy and menstrual provision). For the women who sew the Days for Girls kits, confidence and agency are also gained through income and skills-education. The strengths and challenges of Partners' programme reveal the importance of menstrual literacy education, the use of women's knowledge in NGO work with women, and a whole-of-community response to menstruation needs. The research also informs wider understandings of how MHM discourse and development practice affects menstrual stigma. 


\section{Acknowledgements}

My earnest thanks to the community that made this work possible:

To Sara, for your endless hours reviewing, suggesting and nurturing. Your wisdom and experience always inspire me to think and care deeply. Thank you for sharing your life and yourself with me and allowing me to share with you.

To Penny, Courtney and Lena, for taking time to proofread chapters. You recognised me in my stressful state and offered your generous help - thank you.

To Dan - for your love, always. And a special thanks for your support during these last crazy months, taking on my burdens and allowing me the space to work.

To my family and my church family. You are the rock this thesis is built upon. You taught me how to love, listen and serve.

To my dear friend, Rose, for the chats, company and solidarity you offer. Despite not being geographically present you are always emotionally so, and your curiosity about my topic has been meaningful motivation for me.

ขอบคุณค่ะ to the team at Partners Relief and Development, for your generous welcome and endless accommodation. I left Thailand inspired by the way that you work tirelessly for so many people.

Thank you to the New Zealand Ministry of Foreign Affairs and Trade, for the Field Research scholarship that enabled my trip to Thailand for this work.

Lastly, my thanks to each woman that participated in this research - students, migrant women of Mae Sot, the talented women who sew DfG kits and Partners staff. You shared with me your stories of hardship and joy, community and womanhood and I am honoured to have learnt from you. 


\section{Contents}

Abstract .............................................................................................................................................................................

Acknowledgements ........................................................................................................................................................iii

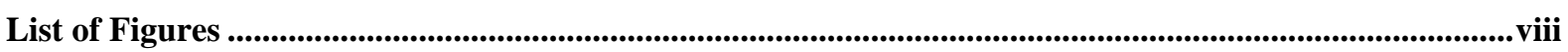

Chapter 1 - Introduction .............................................................................................................................................. 1

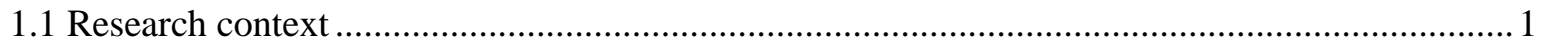

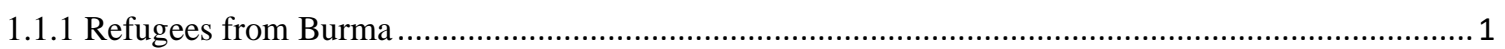

1.1.2 Menstrual Health Management relief...................................................................................

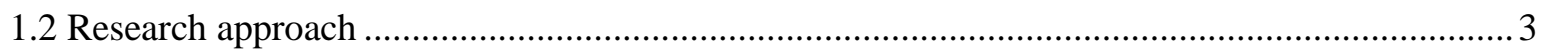

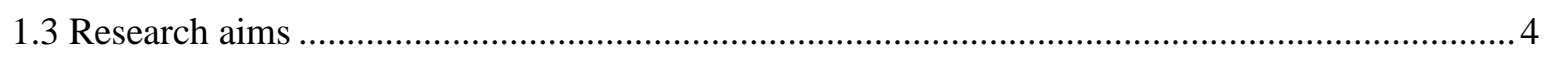

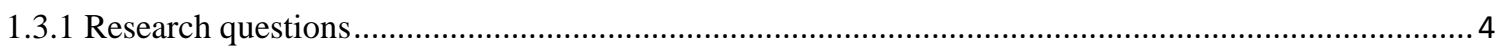

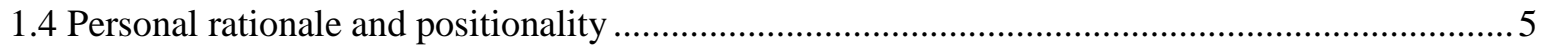

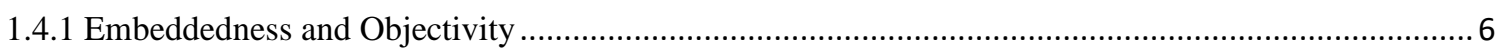

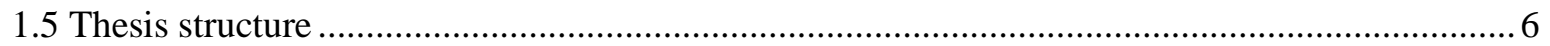

Chapter 2 - Menstrual Health Management...........................................................................................................8

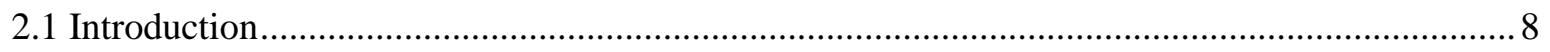

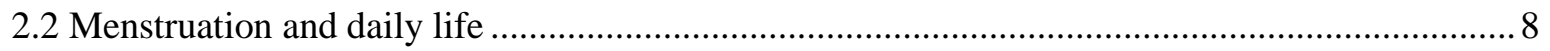

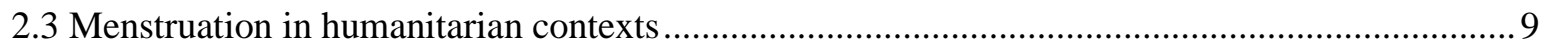

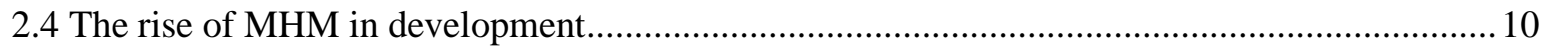

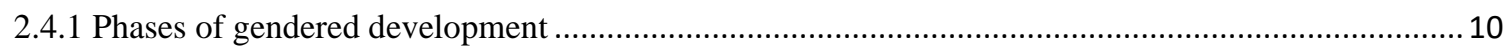

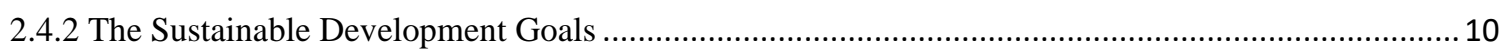

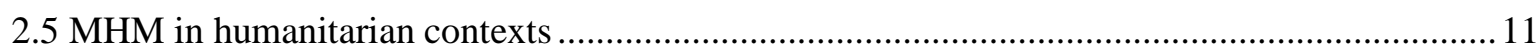

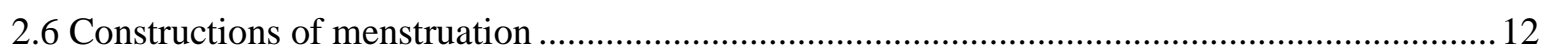

2.7 Critique and suggestions for MHM from the literature ….................................................. 13

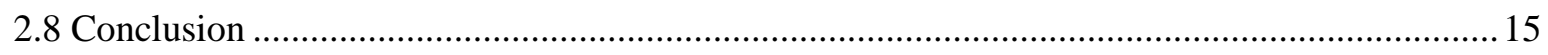

Chapter 3 - Geopolitical Context ....................................................................................................................16

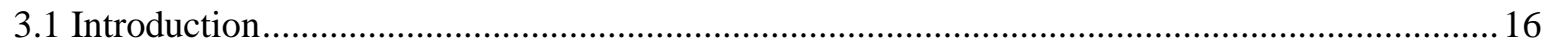

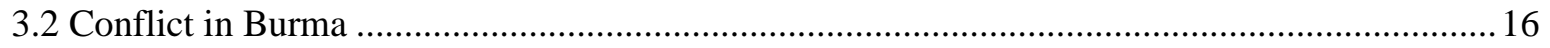

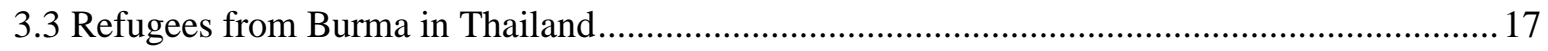

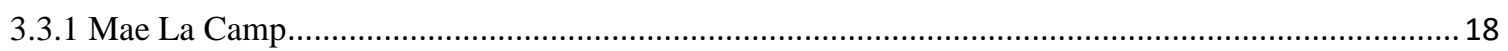

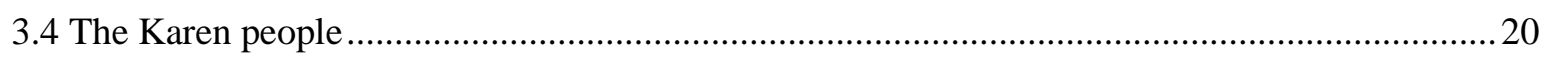

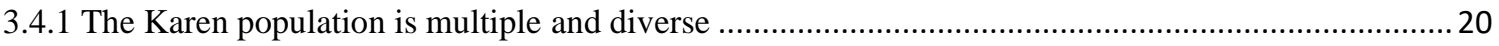

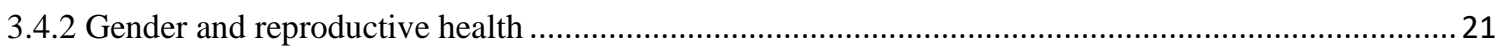

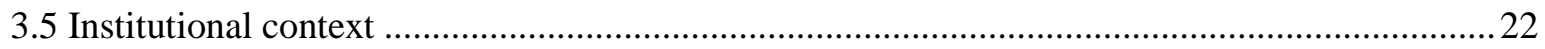

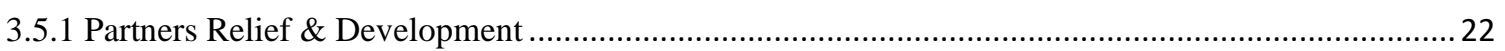

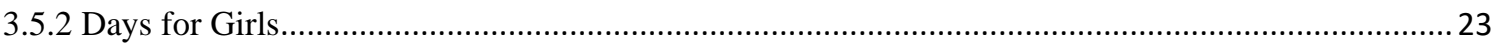




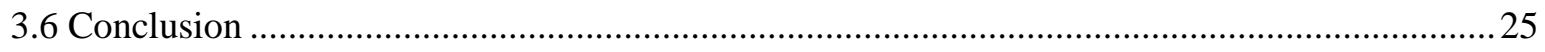

Chapter 4 - Research Approach .....................................................................................................................................26

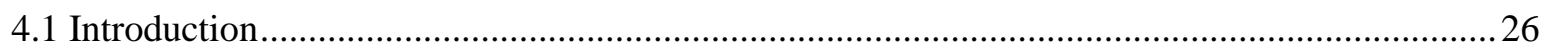

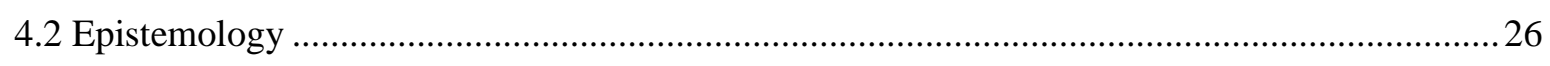

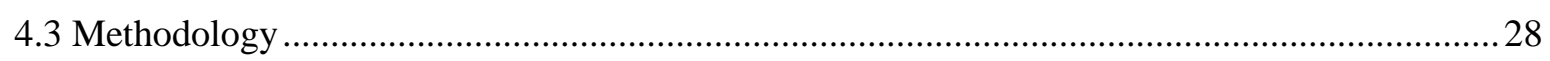

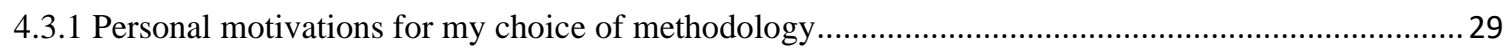

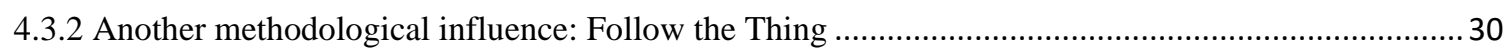

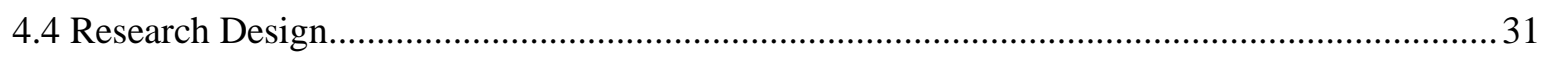

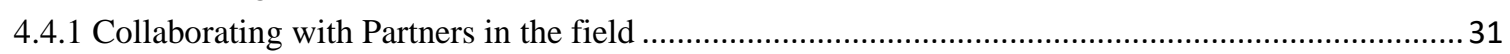

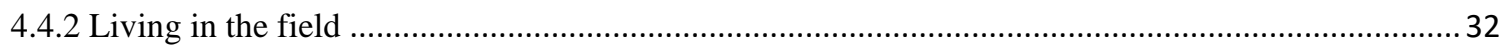

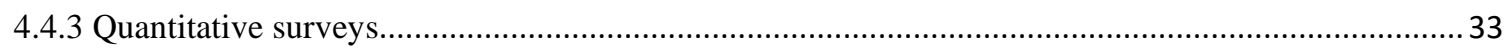

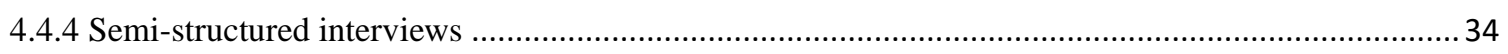

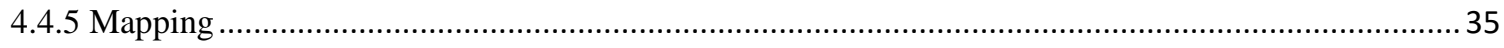

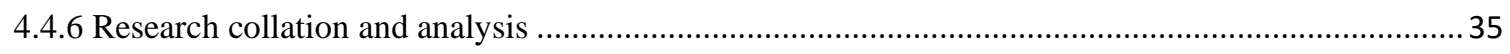

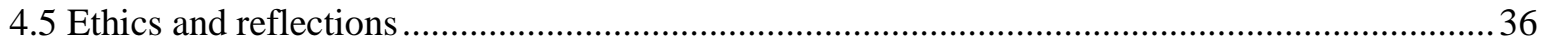

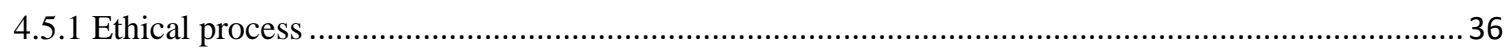

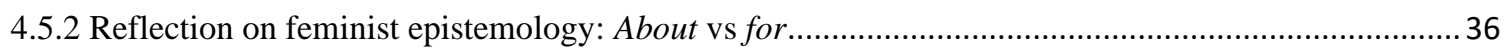

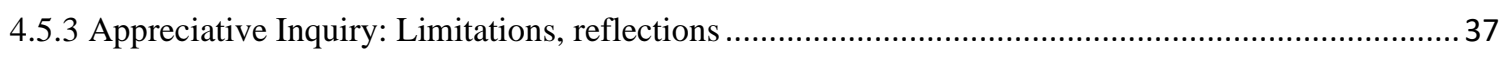

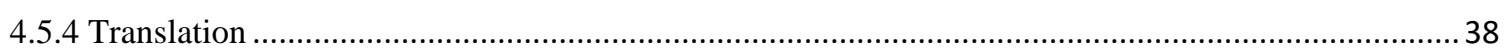

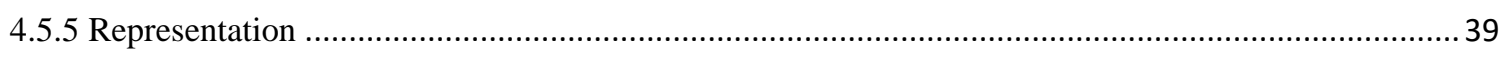

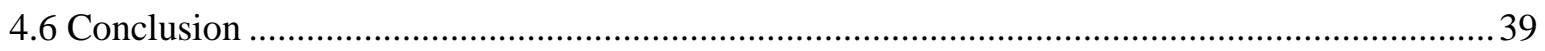

Chapter 5 - The MHM Programme ............................................................................................................................. 40

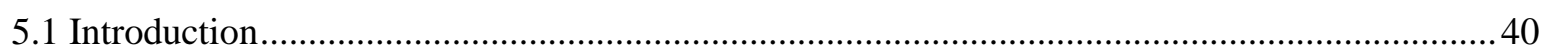

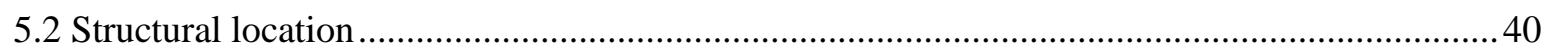

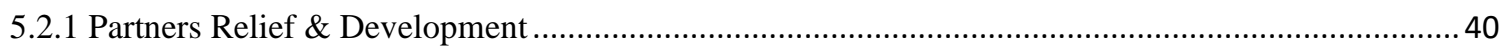

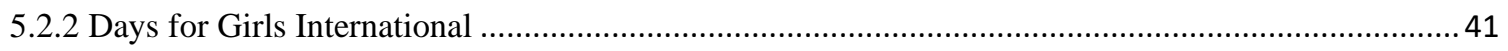

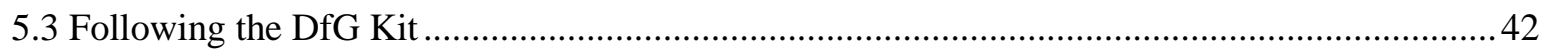

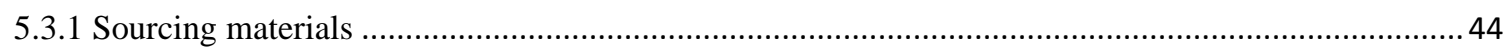

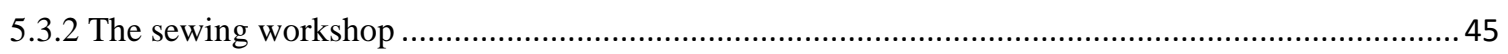

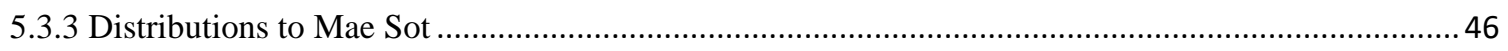

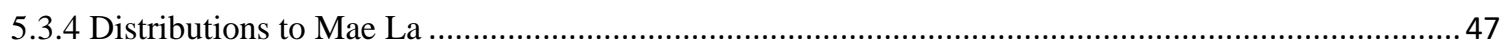

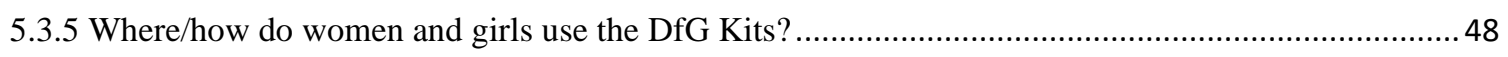

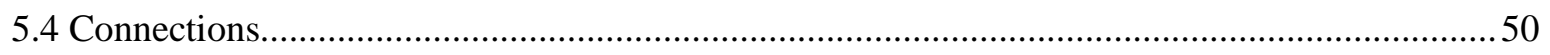

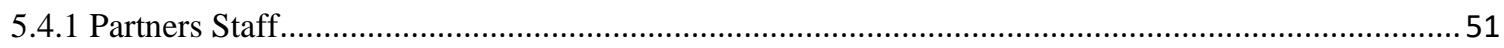

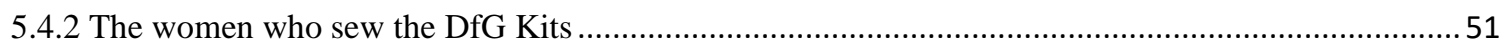

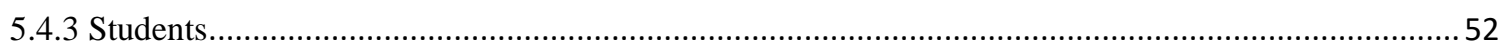

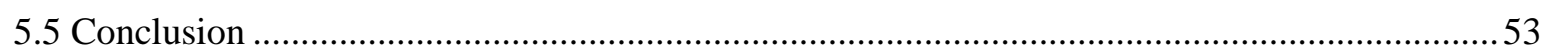

Chapter 6 - Kit uptake and effects............................................................................................................................55

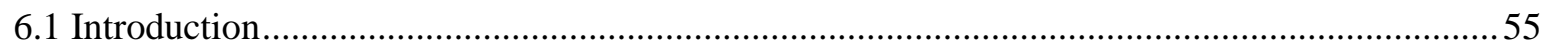




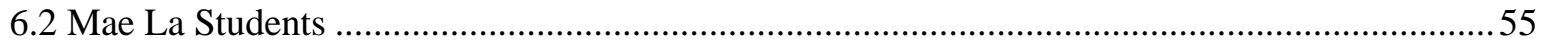

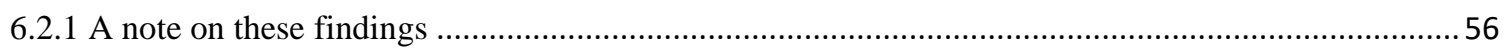

6.2.2 What did students use before they received the DfG Kit? .......................................................... 57

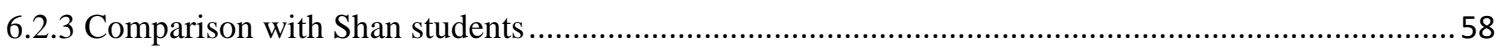

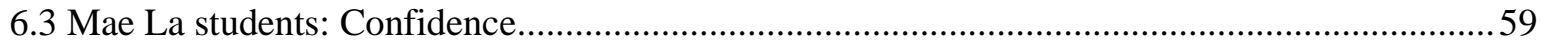

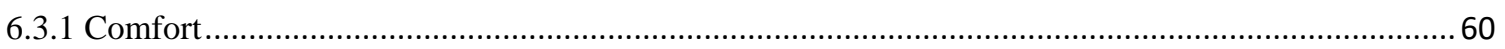

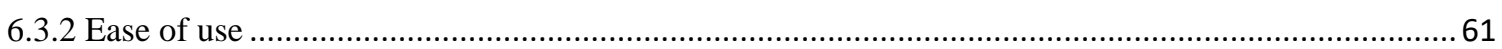

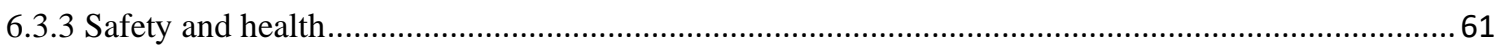

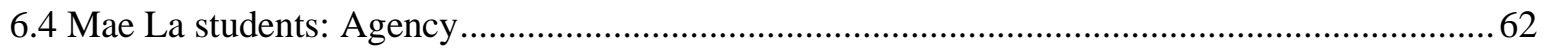

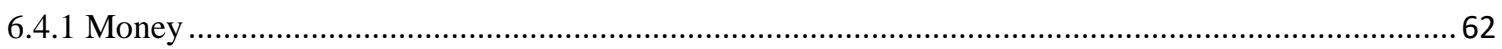

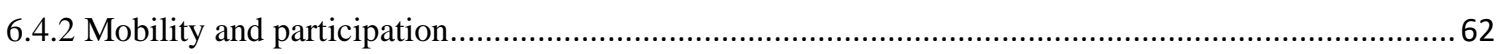

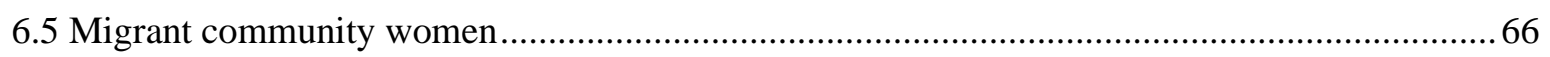

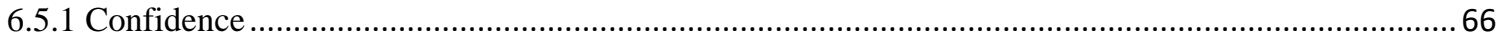

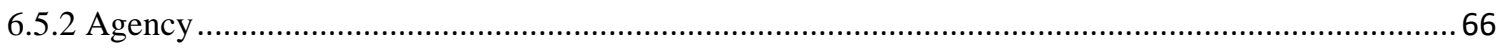

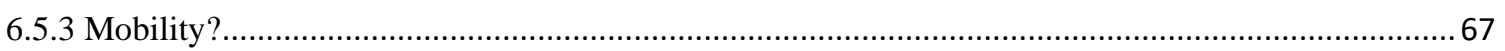

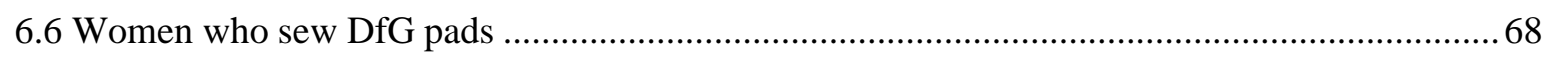

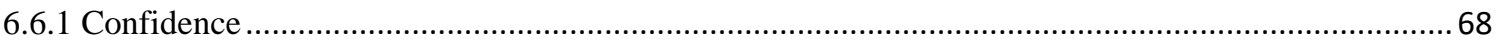

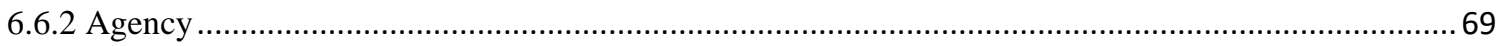

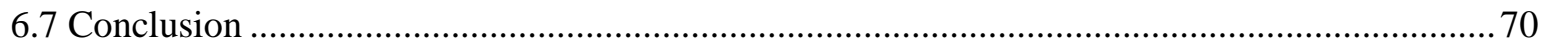

Chapter 7 - Knowledge ..................................................................................................................................... 71

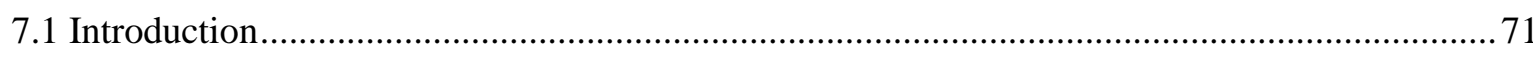

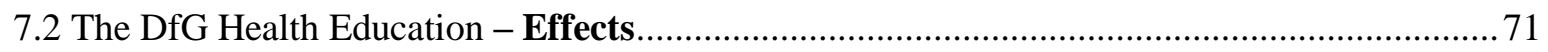

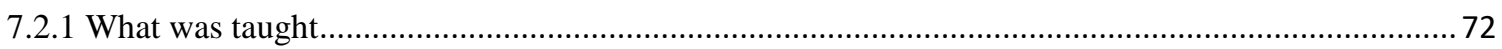

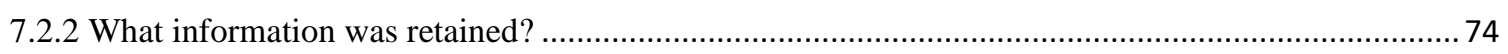

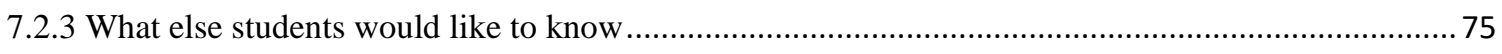

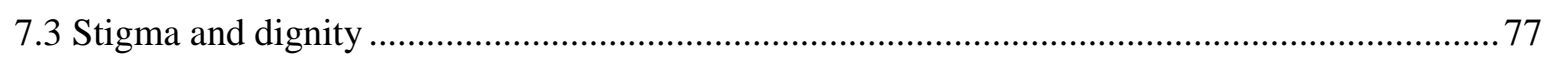

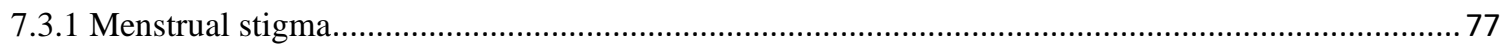

7.3.2 How the programme has helped to combat menstrual stigma........................................................ 78

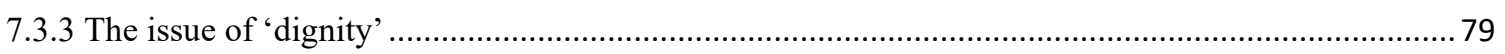

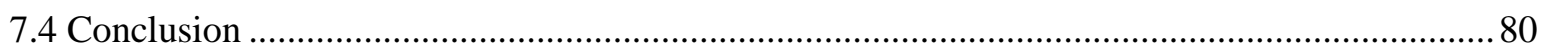

Chapter 8 - Analysis of the Partners MHM Programme ...............................................................................8 81

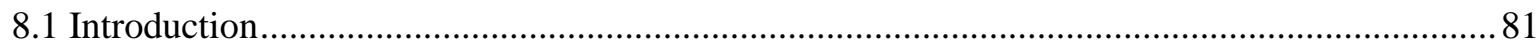

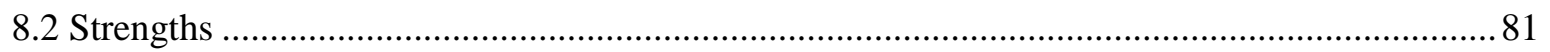

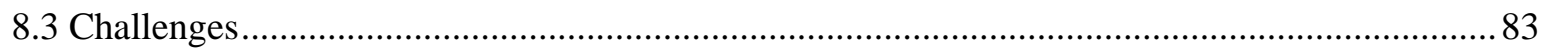

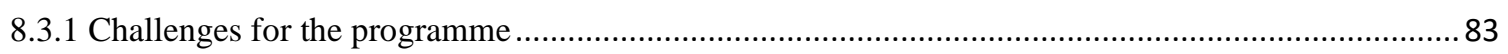

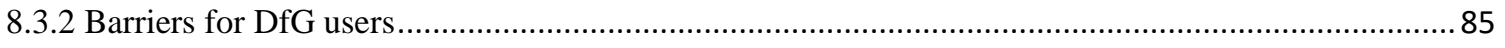

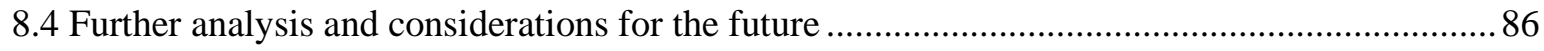

8.4.1 How does Partners learn from the communities it serves? ............................................................ 86

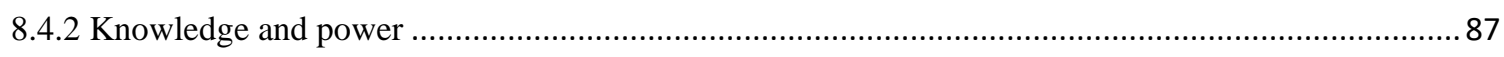

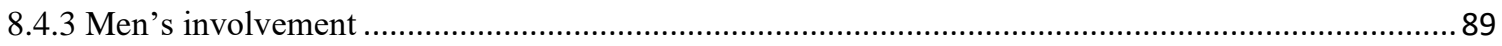


8.5 Conclusion 91

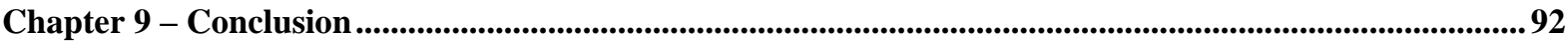

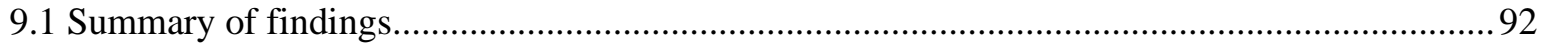

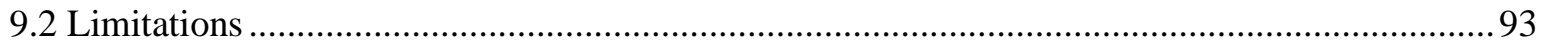

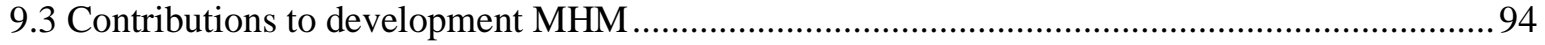

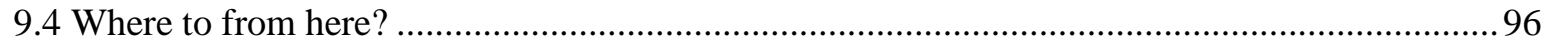

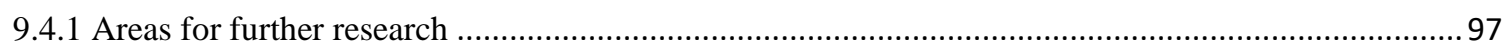

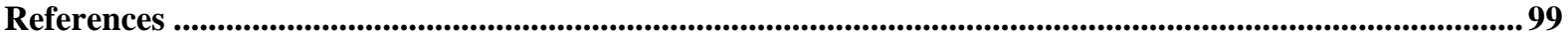

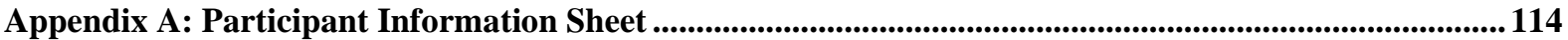

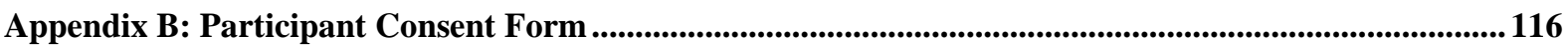

Appendix C: Question Guide Example.........................................................................................................117

Appendix D: Translator Agreement .................................................................................................................... 118 


\section{List of Figures}

3.1 Refugee and IDP Camps along the Thailand-Burma Border .......................................... 18

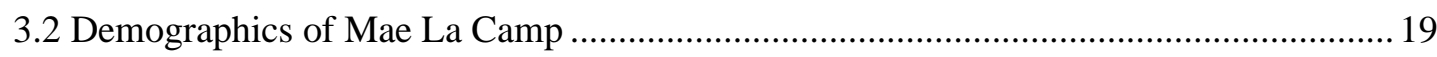

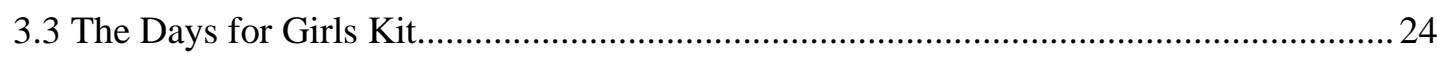

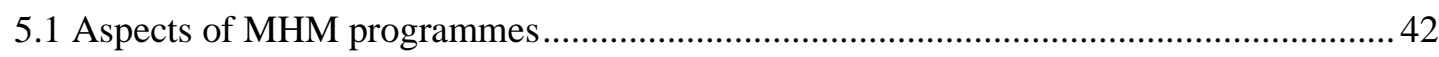

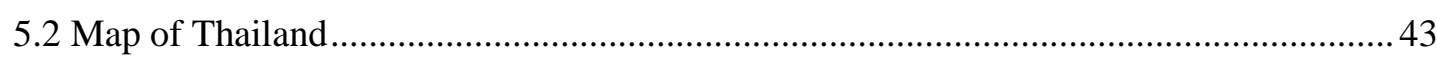

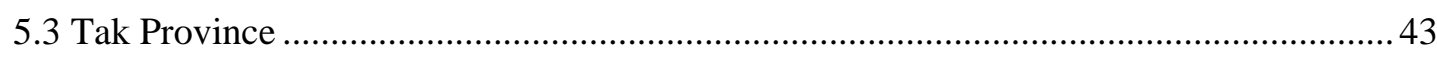

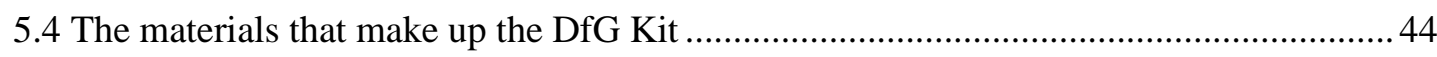

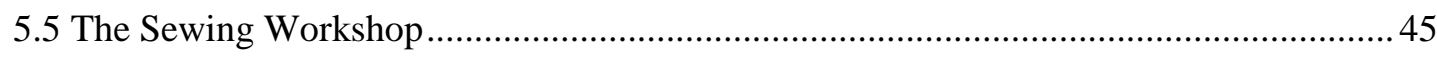

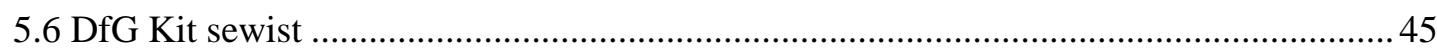

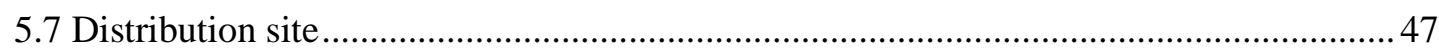

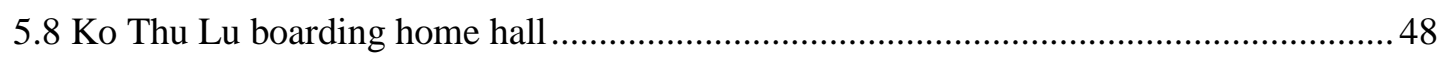

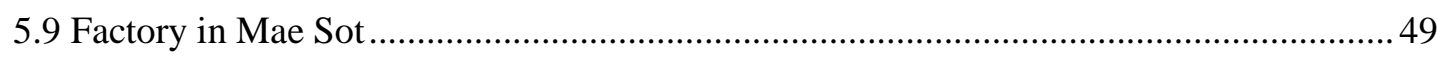

5.10 Washing and drying space in Ko Thu Lu boarding home..........................................50

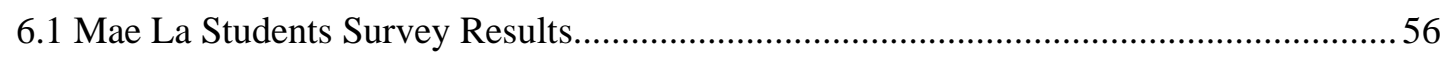

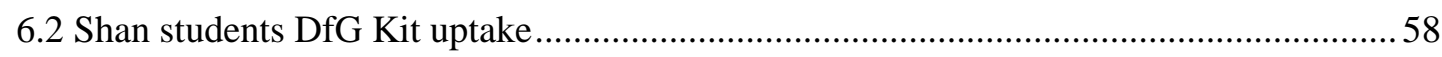

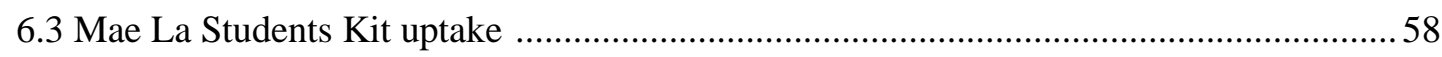

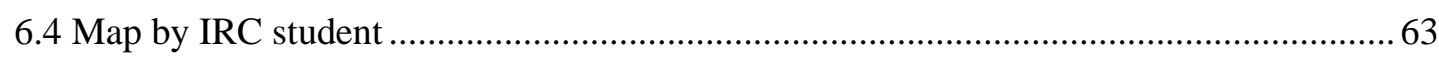

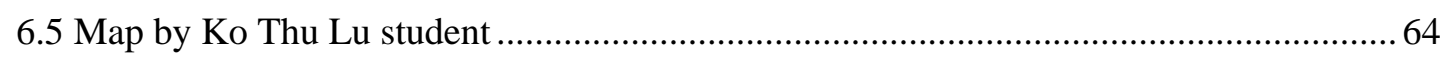

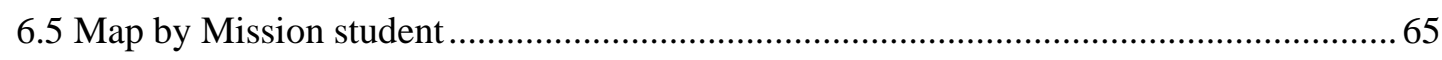

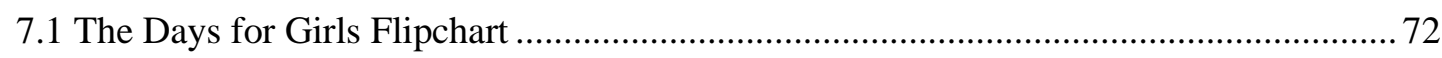

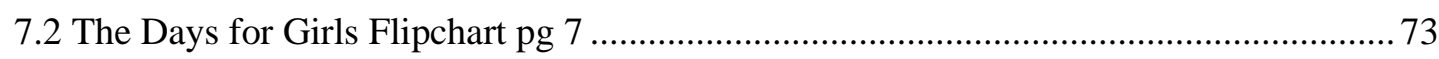

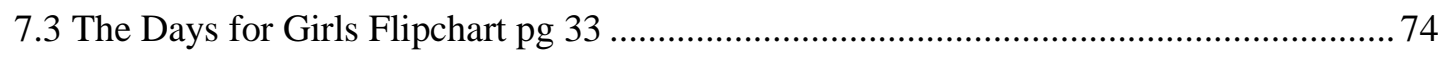




\section{Chapter 1 - Introduction}

\subsection{Research context}

Menstruation is a process that affects more than half of the human bodies in our world. For many, it is a process laced with ideas of shame and uncleanliness. For others it is seen as beautiful, feminine and life-giving. Recently, menstruation has become the subject of feminist, public health and development movements that aim to reduce menstrual stigma and alleviate "period poverty" through the provision of menstrual products (Dawkins, 2018; Dignity, 2019; George, 2017). For women and girls ${ }^{1}$ that have been displaced by conflict and emergencies, their precarious living situations can inhibit access to menstrual products and education (Benner et al., 2010; Parker et al., 2014). This "period poverty" has been linked with increased experiences of stigma towards blood leaks which can affect women's confidence and agency.

Since the early 2000s, global humanitarian emergency response actors have increased their focus on Menstrual Hygiene Management (MHM) relief in post-disaster and post-conflict settings. For women living in refugee camps, difficulties during menstruation can be heightened due to scarcity of menstrual resources, disruption of support networks and lack of private and safe facilities (Kågesten et al., 2017; Parker et al., 2014). Improving menstrual health outcomes for women and girls is understood to allow them confidence and to increase their religious, social and educational participation (Clatworthy et al., 2018; Dolan et al., 2012; Dolan et al., 2016; Hennegan et al., 2017). Therefore, interventions to this end are thought to improve confidence and agency for women facing the challenges of displacement. This thesis explores how processes of Menstrual Hygiene Management relief contribute to increasing confidence and agency for refugee women on the Thai-Burma border. It is a 'case study example' that also feeds into MHM discourse around menstrual bodies, stigma and the role of menstrual health education.

\subsubsection{Refugees from Burma}

It is estimated that close to one million people have been displaced due to ongoing conflict and civil war in Burma (OCHA, 2019). Of this number, 93,000 people are living in nine refugee camps in neighbouring country, Thailand (UNHCR, 2019). Having fled over the Thai border, refugees from several ethnicities have sought to live in camps and migrant settlements hoping to escape the violence at home and to begin new resettlement processes. However, resettlement numbers are relatively low: only 2,769 refugees from Thai refugee camps were resettled in 2018 (IOM, 2019). As a result, thousands

\footnotetext{
${ }^{1}$ This thesis refers menstruators as 'women' or 'women and girls' to be in line with and relevant to MHM practitioners and the participants in this study who use this language. However, I acknowledge that not all girls and women menstruate, and not all menstruators are women and girls.
} 
of refugees from Burma have lived confined to the camps in Thailand for over 30 years. Life in these spaces is far more permanent than transient and the lines between 'camp' and 'settlement' have become blurred (Keenan, 2019).

The largest refugee camp on the Thai border is Mae La, home to more than 37,000 refugees (The Border Consortium, 2019). While over $90 \%$ of the population come from Karen State, other ethnicities include Mon and Bamar. Religious backgrounds of residents are diverse, including Buddhism, Christianity and Animism. Bartholomew \& Calder (2018) report that cultural and religious values affect customs around menstruation, and that while there is a general stigma and taboo surrounding the topic, there is a wide variation of menstrual health practices. This diversity makes the development and practice of appropriate interventions complex and worthy of further investigation.

\subsubsection{Menstrual Health Management relief}

Buthathoki et al. (2018) describes adequate MHM as, "clean menstrual management material” that can be changed and cleaned in privacy as often as necessary, "using soap and water for washing the body as required, and having access to facilities to dispose of used menstrual management materials" (p. 2). Programmes designed to achieve these outcomes are usually carried out by NGOs and include the provision of sanitary pads (usually cloth and reusable), education on menstrual health, provision of, or advocacy for, appropriate washing facilities, and safe, private toilets.

There is a growing body of literature concerned that large proportions of girls who have reached menarche (first menstruation) lack knowledge of menstrual health and access to sanitary care (Ritu, 2017). Furthermore, low access to MHM products and poor menstrual knowledge have been proposed as barriers to health and school attendance (Ritu, 2017). In response, development and humanitarian actors in a range of contexts have been conducting programmes to target these needs.

One such organisation is Partners Relief \& Development (AKA 'Partners'), an international NGO based in Chiang Mai, Thailand. Partners is a Christian aid agency whose 'humanitarian relief' sector includes the provision of basic essentials to those in refugee camps in Burma and on the Thai border. For sanitary health, Partners distributes 'Days for Girls' (including reusable menstrual pads, underwear and soap) kits to women and girls along with menstrual health education (J. Bardsley, 2018). The kits are sewn by women from Burma in a workshop run by Partners on the Thai-side of the border.

MHM relief has been shown to positively affect mobility, confidence and agency for women and girls, important factors in contemporary Development Studies. It can increase girls' ability to access education and women and girls' ability to partake in daily community activities (Clatworthy et al., 2018; Dolan et al., 2012; Dolan et al., 2016). MHM relief can also help women and girls to feel confident in their abilities to deal with their periods and to experience decreased levels of anxiety and shame 
(Mucherah \& Thomas, 2017). Women can attain more confidence and agency around MHM when they are able to input their knowledge of cultural and humanitarian contexts (Sommer et al., 2017).

\subsection{Research approach}

The research for this thesis is informed by a feminist epistemology. This orientation promotes inquiry that is focused on outcomes for women, which fits well with the aims of MHM research. The feminist approach encourages representation of marginalised voices and recognition of diversity (O'Leary, 2017). Furthermore, feminist epistemology seeks to understand and challenge cultural norms (O'Leary, 2017), so it allowed me to gather a deeper knowledge of how culture and development movements inform menstrual health perceptions and practices.

A feminist orientation considers how female participants are affected by the research itself (Castree, Kitchin \& Rogers, 2013). It challenges researcher-researched power dynamics of traditional Western study, prompting me to consider where MHM knowledge is held and how my research can avoid being deficit-based and extractive. Consequently, I opted to take an appreciative approach which aimed to value the efforts of Partners and the input and knowledge of the women for whom they work. Previous studies in humanitarian MHM have largely been deficit-based and centred around the difficulties of the relief situation. However, development research has been shifting since the 1970s and approaches such as Participatory Research and strengths-based feminist research have focused more on the assets of the participants involved than the challenges they face (Black, 2003; Chambers, 1994; Pollio et al., 1997). In line with this development, I have chosen to inform my research with principles and processes from 'Appreciative Inquiry'- a strengths-based framework with an emphasis on appreciating the value of an activity rather than its problems (Reed, 2007). This means that my questions were directed toward "appreciating the activities and responses of people, rather than concentrating on their problems." (Reed, 2007, p. 2). While Appreciative Inquiry is based on four stages of action - Discovery, Dreaming, Design and Delivery, for the purposes of this research I focus on Discovery (appreciating the strengths) and Dreaming (envisioning what might be).

The research adopted a mixed-methods methodology comprised of semi-structured interviews, quantitative surveys, visual mapping and field observation. Semi-structured interviews were carried out with Partners staff, women in Thailand who sew the Days for Girls Kits, and women residents of Mae La Camp. These interviews were led by the ideas of 'Discovery' and 'Dreaming' within Appreciative Inquiry (Cooperider \& Whitney, 2001) to gain a deeper understanding of MHM experiences and how they perceived the work of Partners and the provision of Days for Girls Kits. Interviews with students also involved a 'mapping' exercise - a visual method that I hoped would convey their voices more effectively across communication barriers. For a quantitative understanding of MHM impact, I used a structured interview-survey in four classrooms of female students in Mae La Refugee Camp boarding 
homes. Additionally, I carried out field observation to provide contextual information and to enable me to reflect on my own understandings and biases during the research.

Findings were analysed using holistic thematic analysis (Reissman, 2005). Firstly, I conducted an inductive analysis to search for recurrent themes that emerged across the interviews, surveys and fieldnotes. I looked especially for participants' own ideas of improvements to MHM in the camp in the future. I then took a more deductive approach, with a focus on the key questions I developed through exploratory and secondary research, and triangulation of findings with secondary data and literature.

\subsection{Research aims}

Research on humanitarian relief is a fast-growing field within Development Studies. Humanitarian relief, generally defined as "impartial, neutral, and independent provision of relief to victims of conflict and natural disasters," is often far from impartial and simple, and is increasingly being held to the standards of international development goals (Barnett et al., 2008, p. 7). Outcomes from the most recent World Humanitarian Summit reflect the 2015 Sustainable Development Goals in their focus on not only the provision of food and water, but measures that will allow "people's safety, dignity and the right to thrive" (OCHA, 2018, p. 15).

The field of Menstrual Health Management relief strives to promote these ideas. Through the provision of menstrual products, appropriate toilet facilities and menstrual hygiene education, relief organisations in recent decades are providing tools so that women and girls in disaster situations and refugee camps can manage their periods and increase their mobility. But in the insecurity of humanitarian situations, best-practice relief can be difficult. In response, some MHM practitioners are attempting to understand the ways in which they can improve their systems. This research is a 'case study example' of how humanitarian MHM care can be effective and how those involved in and benefiting from MHM processes might further improve women's safety, dignity (or confidence) and right to thrive.

\subsubsection{Research questions}

1. How can public perceptions of menstruation be affected by discourse and menstrual literacy education?

While academic literature on menstrual cultural values for specific Burma ethnicities is hard to obtain, a 2018 report by Bartholomew and Calder outlined some general trends. Many girls felt ashamed about their menstruation, and there was a general discourse that menstruation was 'dirty'. It was normal for girls to be told to avoid some daily practices during menstruation, including washing their hair, spending time with boys, eating spicy food and playing or running around (Bartholomew \& Calder, 2018). Additionally, Western NGO discourse around menstruation can reproduce menstrual stigma by overemphasising the importance of 'covering up' blood with menstrual products in our "culture of concealment" (Houppert, 1999). Through Question 1, I aimed to understand how ideas of dignity and 
shame are perpetuated both culturally and structurally - and how menstrual literacy education and discourse can replace shame with confidence.

\section{How does Partners' Menstrual Health Management (MHM) relief affect confidence and agency for} refugee women on the Thai-Burma border?

Using a mixed-methods methodology informed by Appreciative Inquiry, and through the perspectives of the women involved, I aimed to identify the strengths of the programme that have implications for women's confidence and agency in MHM. The research looked to see how Partners' MHM programme (called 'Days for Girls' or 'DfG') has learnt from, or could learn from women's cultural, practical and situated knowledge about menstruation and MHM. I identified areas where improvements, whether practical or ideological, could be made to better support women living in these camps. I also looked to how women contribute to the outcomes of confidence and agency.

3. How can lessons learned from this case study inform wider understandings of MHM in development?

There is limited analysis of how non-government organisations (NGOs) incorporate women's knowledge, how interventions are received in specific cultural contexts, and what their effects may be on women and girls' participation and empowerment. Academically, this study goes some way towards filling the gap in humanitarian MHM literature. This research also aimed to identify successful MHM initiatives for women from Burma that could perhaps inform future humanitarian MHM practices.

\subsection{Personal rationale and positionality}

This research project evolved as a connection between my ongoing professional and research interests in diverse refugee journeys, my interest in NGO practice, and a growing awareness of menstruation as a symbol of dignity and agency. It was also motivated by my faith background, which encourages me to love, serve and learn from people who are different to me, whether that difference is cultural, social or situational.

In 2017, I spent time in a refugee camp in Greece as a volunteer with Refugee Support Europe. I worked in a small grocery shop, the camp kitchen and in a classroom teaching art. The residents shared with me their journeys to the camp, and the importance of camp spaces allowing participation in 'daily life' activities as much as possible. I was challenged by their stories and on my return, I reoriented the focus of my undergraduate degree towards understanding more of the diverse experiences of refugees. During my Honours degree, I studied the employment resettlement experiences of former refugees and I began work in a homework club for students from refugee backgrounds.

Five years ago, my family visited Partners Relief \& Development in Thailand and learnt about the work they do with refugee and migrant communities. We learnt of the impact of ongoing conflict on families and communities from Burma and the permanency and difficulty of life in Thai border camps. Years 
later, my parents and sister decided to work for this NGO, and it was from them that I learnt about a need and an opportunity for research in their menstrual hygiene programme. As I already had an interest in this area, I was excited to take on the subject as my research topic and learn more about humanitarian MHM.

It important to acknowledge that I am not from a refugee background and despite my learning, I have little knowledge of what it is like to have to flee from home because of persecution. I am advantaged socio-economically, both in my home of New Zealand and when compared with people in Thailand, and I have freedom of movement. However, like the women from Burma with whom I researched, I do know what it is like to pursue education and daily activities while navigating the cultural and practical difficulties of having a period.

\subsubsection{Embeddedness and Objectivity}

I also acknowledge that my personal connections to Partners Relief \& Development affect how I have researched with them. In many ways, I see this as a strength. Feminist scholar Donna Haraway (1991) states that partiality of vision can lead to greater insight for analysis because 'situated knowledge' benefits from relationships within community. In my case, my connections mean I already have longterm background knowledge of Partners' work which have aided the passion and motivation of the NGO staff. Furthermore, my relational embeddedness has been valuable to my research because it encouraged me to research carefully around such a sensitive topic. Haraway calls this situated knowledge "feminist objectivity" - where research takes a more responsible standpoint when we acknowledge the contingency of our position in the world, rather than claiming a neutral viewpoint: "Feminist objectivity is about limited location and situated knowledge, not about transcendence and splitting of subject and object. It allows us to become answerable for what we learn how to see" (Haraway, 1988, p. 583).

I was also motivated to maintain good relationships with the members of Partners and all those they work with, and this has in part inspired my use of an Appreciative Inquiry framework. I have aimed to draw on the strengths of the workers and the recipients of the MHM programme I am studying.

However, embracing partiality does not rule out striving for integrity in my research. I have been careful to analyse my preconceived assumptions about Partners' work and to stay open to criticism and honest opinions that challenged my assumptions from all of those I interviewed.

\subsection{Thesis structure}

Following the introduction, this thesis sets the foundations of the research in an overview of Menstrual Health Management (Chapter Two). Here, I review the theory and literature on MHM, highlighting some gaps that will be explored in this thesis. Chapter Three is a description of the geopolitical contexts 
of the research. I specifically look at aspects of gender and displacement for Karen people before exploring the institutional and NGO context. Chapter Four deals with my research approach including epistemology, methodology and methods. I interweave reflections and ethical considerations throughout this section.

The analysis chapters delve into the Partners MHM Programme, the stories of its participants and how the programme has affected their lives. These findings are linked, where appropriate, to other literature and studies. The organisation of these chapters considers aspects of Appreciative Inquiry, beginning with 'Discovery' of the programme and processes as well as the themes that arose from participants' stories and then moving to understand their 'Dreaming' for the MHM programme. And just as the personal and procedural aspects of the Partners MHM programme are relational and intertwined, the concepts explored within these chapters overlap and inform each other.

Chapter 5 addresses my first research question, tracking the story of the Days for Girls menstrual kit to understand how women contribute to meaningful MHM outcomes for female refugees living in Burma/Thai border camps. Next, Chapter Six takes an evaluative view, presenting quantitative and qualitative findings related to DfG Kit uptake and effects. Chapter Seven then considers the programme and MHM as a whole more deeply, analysing their knowledge-based effects, especially exploring ideas of dignity and shame. A further analysis of the programme is carried out in Chapter Eight, focusing on the strengths of the programme and possible areas for growth. It ends by presenting the Dreams for the programme by the women who have been a part of it - staff, sewists and participants.

In Chapter Nine, the Conclusion, I summarise the findings of the research, reflecting on how they answer the aims and how they interact with other literature. I then refer to the 'Design' stage of Appreciative Inquiry - I suggest outcomes for MHM development practice and consider how the findings of this research point to ways that alternative framings and further research could enhance outcomes in MHM for women and girls. 


\section{Chapter 2 - Menstrual Health Management}

\subsection{Introduction}

Literature relevant to Menstrual Health Management seems to be divided into two discourses: NGOs and practitioners tend to see MHM as a practical need to be filled, while academics of feminist theory emphasise that MHM is indicative of, and deeply affected by, wider social and cultural systems.

This chapter attempts to briefly address the two forms of knowledge. It begins with a brief look at menstruation and its place in development and humanitarian contexts. There is an evaluation of MHM's development alongside the Sustainable Development Goals and a review of how humanitarian MHM interventions have been evaluated. I then consider feminist perspectives and touch on some social constructions of menstruation that affect women and girls before concluding with a justification for this research project considering the existing literature.

\subsection{Menstruation and daily life}

In many cultures, framings of menstruation can be positive and menarche can be a culturally important time of coming "into womanhood" (Bobel, 2019, p. 49). But menstruation can also be a hard time for women and girls as they navigate cultural and practical boundaries of bleeding for several days per month. Menstruating women face "cultural stigma and the fear of leaks, lack of knowledge, or lack of menstrual materials" which make being in public difficult (Perczunska \& Preiss, 2014, para. 4). In New Zealand for example, it has been found that culturally discursive practices such as euphemisms and notions of cleanliness and hygiene create representations that stigmatise and devalue menstruation. As a result, young women face a journey of menstruation that often includes "the emotional themes of embarrassment, anxiety and ambivalence" (Culling, 2001, ii).

Many women are "likely to limit their activities during menstruation" which in some contexts, includes "refraining from religious worship and... avoiding men and boys" (Ritu, 2017, p. 4). Menstruation has also been linked to education access by many organisations (Days for Girls International, 2019a; Girl Effect, 2008; UNESCO, 2014). In Sierra Leone, a study showed that over $20 \%$ of female students surveyed missed school during their periods. This percentage was 30\% in studies from Afghanistan and Nepal. Close to quarter of female students in India have been said to drop out of school when they begin menstruation, while girls that remain miss five days a month on average (Perczunska \& Preiss, 2014). At the same time, many admit difficulty in securing a relationship between MHM and school attendance, "partly due to the difficulties in measuring absenteeism and its causes, especially when linked to menstruation.” (UNESCO, 2014, p. 15). 
What has been agreed is that there are several factors involved, aside from the provision of menstrual products. Potential surrounding problems for school attendance are lack of safe water, sanitation and safe hygiene (WASH) facilities for girls to be able to change their pads (Bobel, 2019; Hennegan et al., 2017; Sommer et al., 2017). For example, one student in Uganda reported having to take 45 minutes out of her school day to change at home because she was not comfortable using the toilets at school.

\subsection{Menstruation in humanitarian contexts}

In humanitarian emergency contexts, such as disaster zones and refugee camps, difficulties during menstruation can be heightened; "Humanitarian emergencies, which generally include large-scale and long-term displacement, can disrupt family, peer, school, religious and other social support networks factors that are key influences on [...] well-being during adolescence” (Kågesten et al., 2017, p. 10).

The loss of support networks and exposure to insecure environments during forced displacement can make it even more difficult to deal with the normal stress of a period. Furthermore, humanitarian settings (i.e. refugee camps) are often ill-equipped with appropriate resources and facilities for menstrual health management. Issues identified include difficulty of obtaining quality underwear, camps lacking privacy for women to cope with their menstrual periods with 'dignity' and insufficient space to hang reusable cloths in dwellings (Parker et al., 2014). The lack of appropriate facilities for drying undergarments is an issue repeated in many studies (Benner et al., 2010).

A study conducted with internally displaced populations in Burma (Schmitt et al., 2017) reported changes in menstrual hygiene practices among girls and women after displacement. Women usually changed the types of materials (e.g. disposable pads, reusable pads, cloths, etc.) they used according to what was now available to them in their new environment and the ease of using them. Their practices could be affected by the availability of facilities to wash and dry their reusable materials, or by means to dispose of disposable materials. Women also faced challenges relating to toilet facilities such as "large gaps in the bamboo walls permitting visibility, and an absence of locks on the doors" (p. 6). The facilities resulted in anxiety for many women and girls around the potential of being watched or intruded on while using these facilities. This anxiety due to lack of safe and private spaces is an example of how menstrual health issues in humanitarian spaces can affect mental health for refugee women and girls. 


\subsection{The rise of MHM in development}

\subsubsection{Phases of gendered development}

Humanitarian responses to women's menstrual needs have come following the progression of women and gender-focused development. Women in development (WID) began as a development policy in the 1970s in response to predominantly welfare-based approaches of working with women. Instead of framing women solely as wives and mothers, Ester Boserup amongst other feminists pushed a focus on women's roles as economic actors in development (Saunders, 2002). At this time, however, menstruation was not anywhere near the forefront of 'development' thinking. The WID approach was criticised for its tendency to focus mostly on the productive and money-making aspects of women's work, and too little on the reproductive side of women's lives (Rathgeber, 1990).

In the late 1970s, Women and development (WAD) schools of thought focused more on the relationship between women and development. Dependency Theory feminist scholars criticised the 'modernisation' discourse within WID that focused only on economic improvement. WAD took a more structural understanding - that women have been adversely affected by issues of class and existing development structures (Rathgeber, 1990).

While provision of menstrual products and menstrual health education became more predominant at this time, there was little deeper analysis of gendered and social issues around menstruation. In fact, Sommer et al., (2015, p. 1303) critiqued education and public health actors in development for their avoidance of the issues of menstruation social stigma: "Menstrual shame and the complexities of menstrual management were perceived as an inevitable part of the social order, and other priorities for the limited existing resources consumed attention."

Gender and development (GAD) thinking in the later part of the $20^{\text {th }}$ Century saw feminist scholars engage with gender far more broadly. GAD is concerned with gender relations between both women and men and attempts to address concepts of power in gender (Chant \& Gutmann, 2002; Cornwall, 1997). Within this movement, development practice needed to have "concern for the empowerment of women and for the legitimization of women's knowledge and experience." (Rathgeber, 1990, p. 500). It also aimed to take into account all aspects of women's lives and particularly focus on the social construction of production versus reproduction as the basis of women's oppression (Rathgeber, 1990). While this was the academic theorisation, in practice, a power-critiquing, empowerment-minded approach to menstrual health development did not occur until much later.

\subsubsection{The Sustainable Development Goals}

Current discourse on Menstrual Health Management has shaped and is shaped by the 2015-2030 Sustainable Development Goals (SDGs). The SDGs - "Transforming Our World: The 2030 Agenda for Sustainable Development"-are a development agenda formulated by UN member states and civil 
society members. There are 17 goals, broken down into almost 200 targets, many of which address women and girls' essential place in development. SDG \#5, 'Gender Equality' encapsulates aspects of WID, WAD and GAD thinking:

While the world has achieved progress towards gender equality and women's empowerment under the Millennium Development Goals (including equal access to primary education between girls and boys), women and girls continue to suffer discrimination and violence in every part of the world. [...] Gender equality is not only a fundamental human right, but a necessary foundation for a peaceful, prosperous and sustainable world. [...] Providing women and girls with equal access to education, health care, decent work, and representation in political and economic decision-making processes will fuel sustainable economies and benefit societies and humanity at large. (United Nations, 2015, para. 1-3)

This global concern for narrowing the gender gap in education is said to be where the MHM movement arose. From the education perspective, girls who had adequate MHM may have been able to stay in school (Days for Girls International, 2019a; Girl Effect, 2008)And from the public health perspective, evidence was mounting that educated girls contributed to healthier population outcomes (Sommer et al., 2015). The theme for UN's 2016 Day of the Girl Child was that "Girls' Progress = Goals' Progress" (United Nations, 2016). By that measure, SDG and much of NGO thinking is that "Menstrual Health progress $=$ girls' progress = goals' progress". This discourse, while still current, is beginning to be challenged by MHM literature, as will be explored further in this chapter

\subsection{MHM in humanitarian contexts}

Alongside progress in development, humanitarian response organisations in the last two decades have been increasingly paying attention to addressing the menstrual hygiene management (MHM) needs of women and girls in post-conflict and post-disaster settings. A number of key documents including the United Nations High Commission for Refugees' (UNHCR) Commitments to Refugee Women and Sphere's Humanitarian Charter and Minimum Standards in Disaster Response have emphasised the importance of sanitary provision for women's agency (Sphere, 2000; UNHCR, 2001). The 2011 UNHCR report specifically advocated for providing 'dignity kits' to adolescent girls and women in humanitarian responses, "which frequently include sanitary materials, soap and underwear depending on the local context" (Sommer, 2012, p. 85). Today, a large proportion of aid organisations include MHM in their distributions, and many organisations solely set up to support MHM are providing MHM relief in humanitarian emergencies (Days for Girls International, 2019b; International Federation of Red Cross and Red Crescent Societies, 2018; International Rescue Committee, 2019; Pads4Girls, 2019) 
There are relatively few academic evaluations of humanitarian MHM interventions, likely due to the difficulty of comprehensive research in serious emergency situations. However, a review of pilot testing of the "Menstrual Hygiene Management in Emergencies Toolkit" in three camps hosting Congolese and Burundian refugees in Tanzania showed largely positive effects (Clatworthy et al., 2018). The toolkit programme was established by the International Rescue Committee and Columbia University's Mailman School of Public Health. Under it, reusable sanitary materials alongside MHM education and improved facilities were provided to women in Tanzanian refugee camps. According to interviews with women, the reusable pads provided were perceived as lowering the chances of blood leaks onto clothing and therefore enhancing mobility during daily community activities.

Other MHM interventions have been reported as facing significant challenges. When reusable pads were provided to internally displaced women in Burma, the infrequency of distributions of laundry soap (to wash the kits) limited ability to sustain their use (Schmitt et al., 2017). In the Bwagariza refugee camp in Burundi, reusable pads left outside to dry were frequently stolen, so women were found to be re-using damp pads that hadn't had enough time to dry indoors (Sommer et al., 2017).

Apart from these limitations, some accounts argue that barriers to good MHM lie with the humanitarian development practitioners, for example "insufficient guidance provided by response staff to beneficiaries on the basics of MHM" and "inadequate cross-sector coordination on the content and timing of MHM responses" (VanLeeuwen \& Torondel, 2018, p. 173). In other cases, there is simply a lack of attention paid to MHM relief, as it is not prioritised in the broader scheme of providing food, water and shelter (Sommer, 2012). Studies call for increased monitoring and evaluation of menstrual hygiene programmes, particularly asking women and girls how they could be more sensitive to their needs (Budhatoki et al., 2017; Sommer, 2017).

\subsection{Constructions of menstruation}

Menstrual practices are deeply embedded in culture (Murphy, 2011; Wall et al., 2018) and MHM interventions for refugee women and girls must take into account their cultural, social and religious customs around menstruation. While academic literature on menstrual cultural values for specific Burma ethnicities is hard to obtain, a 2018 report by Bartholomew and Calder outlined some general trends. Many girls felt ashamed about their menstruation, and there was a general discourse that menstruation was 'dirty'. It was normal for girls to be told to avoid some daily practices during menstruation, including washing their hair, spending time with boys, eating spicy food and playing or running around (Bartholomew \& Calder, 2018).

In Burma, some see this as part of their Buddhist religion - they will not visit temples during menstruation or approach certain holy Buddha images (Shravasti Dhammika, 2019). But this practice is 
not in accordance with those who analyse Buddhist texts. According to them, menstruation through a Buddhist lens is not related to purity/impurity, which can only be determined by 'inner washing' of the mind (Jnanavira, 2016; Shravasti Dhammika, 2019).

Restriction of activity during menstruation may have come about through Christian influences, which have had a significant impact on Eastern Burma. In Leviticus 15:19-23, women were said to be ritually impure during their menses, but most Christians do not hold to that teaching today (Jones, 2018). Rather, most agree that menstrual taboos have been culturally created and re-created not only through religion but through stories, practical experiences and communities' attempts to explain the body and its functions (Baldy, 2017; Druet, 2017). For example, Mai Suitaraw of Chin State said that women were told not to go to church during their period - but she believed this restriction to have evolved from women not having enough underwear, rather than religious notions of impurity (Nyein Ei Ei Htwe, 2016).

\subsection{Critique and suggestions for MHM from the literature}

There is evidence that involvement of refugee women's knowledge in humanitarian aid programmes is a major determiner of their outcomes. Women who are living in refugee camps and post-disaster settings are the best experts on their surrounding facilities, their cultures' MHM customs and knowledge, and how MHM relief programmes are or will be received (Sommer, 2012). They are the holders of the 'situated knowledge' from which visualisation for the future can most responsibly happen (Haraway, 1991). The Menstrual Hygiene Management in Emergencies Toolkit developed by the International Rescue Committee and Columbia University's Mailman School of Public Health states that continuous consultation with beneficiary communities is the best way to ensure that interventions are culturally relevant and helpful (Sommer et al., 2017).

Regarding the "Menstrual Health progress = girls' progress = goals' progress" connection, recent scholarship says that this discourse should be viewed through a critical lens. Chris Bobel, in the first scholarly full-length book written about development MHM, The Managed Body: Developing Girls and Menstrual Health in the Global South (2019) took a heavy critique of the 'girling of development' discourse. She questioned whether there is enough data to suggest such a strong correlation between girls' education and community development. Likewise, Stromquist (2006, p. 24) earlier pointed out the problems of conflating full empowerment with education because "it assumes that the experience and knowledge attained in schooling automatically prepares girls to assess their worth and envisage new possibilities." (p. 24). This critique has been repeated through Anna Vesta Rogers' (2014) study in Cambodia, where she recommended a women's-rights approach rather than a solely education-based approach for women's empowerment. The main message from these studies is that there is more to women's empowerment and community development than school attendance rates. 
Bobel also questioned the fervency of the claims that menstrual product provision on its own is such a catalyst for girls staying in school, noting a lack of clear research in this area. She emphasises the importance of "putting menstrual literacy at the center" quoting that "education is almost always a side dish served up to this main course" (Bobel, 2019, p. 10). With menstrual health education as the "main course", Bobel suggests that girls can be encircled in messages of body positivity and reduction of stigma, which she argues is the greatest issue of menstruation.

There is also critique for the "culture of concealment" that is said to be perpetuated by NGOs carrying out MHM. Houppert (1999) defines this "culture of concealment" as the societal expectations that keep menstruation invisible, so that women's bodies can pass as non-menstrual. The idea is that while MHM programmes aim to alleviate menstrual taboo, they often instead further the stigma of menstrual blood by emphasising the importance of covering it up with the menstrual materials they provide. There is a strand of Feminist Geography that deals with the "'leaky', 'messy' zones between the inside and outside of bodies and their resulting spatial relationships" (Longhurst, 2001, i). Robyn Longhurst (1991) refers to the tendency in many societies to conceal as much as possible women's 'leaky bodies' - that spill bodily liquids beyond the accepted boundaries into spaces they are not welcome. She calls this the "menstrual mandate" - "the expectation that menstruation should be silent and invisible" (p. 9). As I look to how feminist geographers (Lahiri-Dutt, 2014; Longhurst, 2001; Moss, 2002) have studied the fluidity and containment of women's bodies, I briefly examine in Chapter 7 the 'menstrual mandate' and 'containment of messiness' involved in menstrual hygiene management.

There is strong encouragement from Bobel that the key to reducing menstrual stigma is not found solely through the provision of sanitary products (although this is not to say that they should be abandoned) but on the emphasis of education that values and deepens knowledge of the menstrual body. She recommends that development and humanitarian MHM programmes underline menstruation as a naturally occurring biological process.

What a girl needs most is not products (or education about products), but to be freed from the menstrual mandate. This is only possible if she consistently and authentically accesses familial and community support to develop body literacy - the capacity to "read" and understand the body. (p. 10)

The ideal is that women and girls can learn to 'read' how their menstrual cycle affects their individual bodies - including understanding when they might have blood flow, when they are more and less fertile and how their bodies change during menstruation. Learning about these natural biological processes can empower women to understand their menstrual bodies, rather than being ashamed or unsure of them.

Finally, there is a growing agreement that men's involvement in MHM is essential if it is to succeed in empowering women (Bobel, 2019; Mahon, Tripathy \& Singh, 2015; Sommer, 2012). This idea reflects the development theory of Strategic Gender Needs, which include structural and societal change to end 
gender inequality (Molyneux, 1985). In this case, positive structural and community discourse about MHM is thought to end the stigmatisation of menstruation because it is no longer a concealed subject. Bobel also comments on this solution: "To this end, everyone surrounding girls—boys, teachers, family members, religious leaders, policy makers, and so on - needs to challenge menstrual stigma. Girls need to be encircled in 360 degrees of body positivity" (p. 10). In Chapter 8, I briefly ask how men are included in the Partners' MHM programme and discourse around menstruation, in an effort to understand how these things can affect public perceptions of menstruation.

\subsection{Conclusion}

This review of MHM and development literature has explored a wide range of ideas that leave theories, questions and gaps to be explored. I hope to provide a contribution to this literature that is relevant to the context of refugee and migrants from Burma in Thailand, a group with which MHM has not been formally researched. In particular, this research addresses the gap in humanitarian MHM evaluations by exploring the ways that women contribute to a MHM programme (Chapter 5), the menstrual experiences of the women I interviewed and comparing barriers and factors of MHM with those already known (Chapters 6 and 8). I also look to the theories of concealment and stigma as I analyse this example of menstrual discourse in Chapter 7. In Chapter 8, I take on ideas of incorporating women's knowledge and men's involvement and assess the journey of the Partners DfG programme towards these practices. Finally, I recognise that there are few strengths-based pieces of literature on humanitarian menstruation. Even this literature review has had a negative focus - on the hardships of menstruation and some of the flaws of current development and humanitarian practice. In my research, I will be looking to see how Partners' MHM programme has already learnt from, or could learn from, academic, institutional and local knowledge in their work with women and girls in MHM relief. 


\section{Chapter 3 - Geopolitical Context}

\subsection{Introduction}

A 'research context' is not a static setting. Rather, it is a constantly evolving and relational set of factors that by nature cannot be fully described. However, especially in cases where the context is contested and complicated, effort to ground research in local and situational knowledge is essential (Wu, 2014). To understand the stories and the situations of the refugees that will be shared later in this thesis, it is important to understand at least some of where they have come from. Therefore, to contextually emplace the stories and the structures of this thesis, I briefly describe the geographical, political, situational and cultural context to my research.

\subsection{Conflict in Burma ${ }^{2}$}

Burma is ethnically diverse, with several ethnic minorities and over 100 languages and dialects. Administratively, it is split into: divisions of predominantly ethnic Burmans, the majority population; and states which are home to ethnic minorities - Arakan (Rakhine), Chin, Kachin, Karen (Kayin), Karenni (Kayah), Mon, and Shan (Smith, 1999, p. 30). Ethno-political conflict has been a continued reality for the country. Predominantly, this has consisted of an armed struggle between the Burman majority, who have dominated the government, and the minority ethnicities who they have persecuted. Today, the two groups' conflict is based on the minorities' plight for equal rights and self-determination (Rogers, 2012).

In 1948, Burma became independent following a century of occupation by British and Japanese authorities (South, 2008). However, Burma's entry into democracy was fragile and complex, with conflict and insurgency that eventually resulted in a military coup in 1962. This military rule lasted for over half a century (ending in 2011) in which the government have been accused of ethnic cleansing, torture and forced recruitment of child soldiers (Ross, 2015).

For the Karen (AKA Kayin) people, whom this thesis mostly involves, conflict has centred around several military groups: the Karen National Union (KNU), formed after independence, a later offshoot called the Democratic Karen Buddhist Army (DKBA), the Burmese military's 'Tatmadaw', and multiple rebel units (Amnesty International, 1999). Despite several attempts for ceasefire agreements,

\footnotetext{
2 There is ongoing debate over how to refer to Burma/Myanmar appropriately since the country returned to its historic name in 1989 under the ruling military regime (Ross, 2015). Since then, many of those from ethnic minorities have been using 'Burma' as a way to reject the authority of the regime. The participants I spoke to in Thailand told me they also preferred to call the country 'Burma'. In recognition of this choice, this thesis uses 'Burma' rather than 'Myanmar'.
} 
the end of the $20^{\text {th }}$ century was characterised by "village burnings, constant demands for forced labour, looting of food and supplies, and extrajudicial killings at the hands of the military" (Amnesty International, 1999, para. 1). During this time, many individuals and families from Burma's minority states were forced to leave their villages to seek safety, becoming internally displaced people and refugees. This commonly includes parents sending their children, especially boys under 18 years old, over the border into Thailand to avoid conscription into the Tatmadaw army (Ross, 2015).

Today, Burma's government has attempted to initiate a "critical transformation to representative democracy" (USIP, 2018, n.p.). In an end to the military regime, the nation held its first democratic election in more than twenty years in 2010 which, while fraught, led to a dialogue between key politicians towards progress for peace (Rogers, 2012). However, various national and regional tensions continue to threaten the transition. In 2017, Burma security forces began a campaign of ethnic cleansing of the Rohingya Muslims including grave abuses of humanitarian and human rights and forcing more than 730,000 to flee to neighbouring Bangladesh (Human Rights Watch, 2019). In the last year, armed conflict between the Burma Tatmadaw and ethnic armed groups has intensified in Kachin, Shan, and Karen states, fuelled by resource disputes and large-scale development projects. Civilians have been constantly endangered by the military regime's indiscriminate attacks on villages and left increasingly vulnerable by national restrictions on humanitarian aid (Human Rights Watch, 2019).

\subsection{Refugees from Burma in Thailand}

For people from ethnic minorities living in the South-East of Burma, Thailand's border is the most accessible. As seen in Figure 3.1 below, 97,000 people are living in nine refugee camps along the border in Thailand, having fled the violence in their home country (UNHCR, 2019). Many more are undocumented and live outside of camps. Over the last three decades, the Thai government has struggled to regulate the mass migration into the country and this is reflected in their policies for refugee status. According to Saltsman $(2014,461)$, "a lack of coherent policy has led to a series of ad hoc measures that treat different groups of Burmese forced migrants differently, based on ethnicity, when they came to Thailand, and where they settled in Thailand."

For the purposes of this research, I will be referring to each of the participants I interview in Mae La Camp as refugees. They have "been forced to flee [their] country because of persecution, war or violence", which is the United Nations definition of a refugee (UNHCR, 2019, n.p.). 


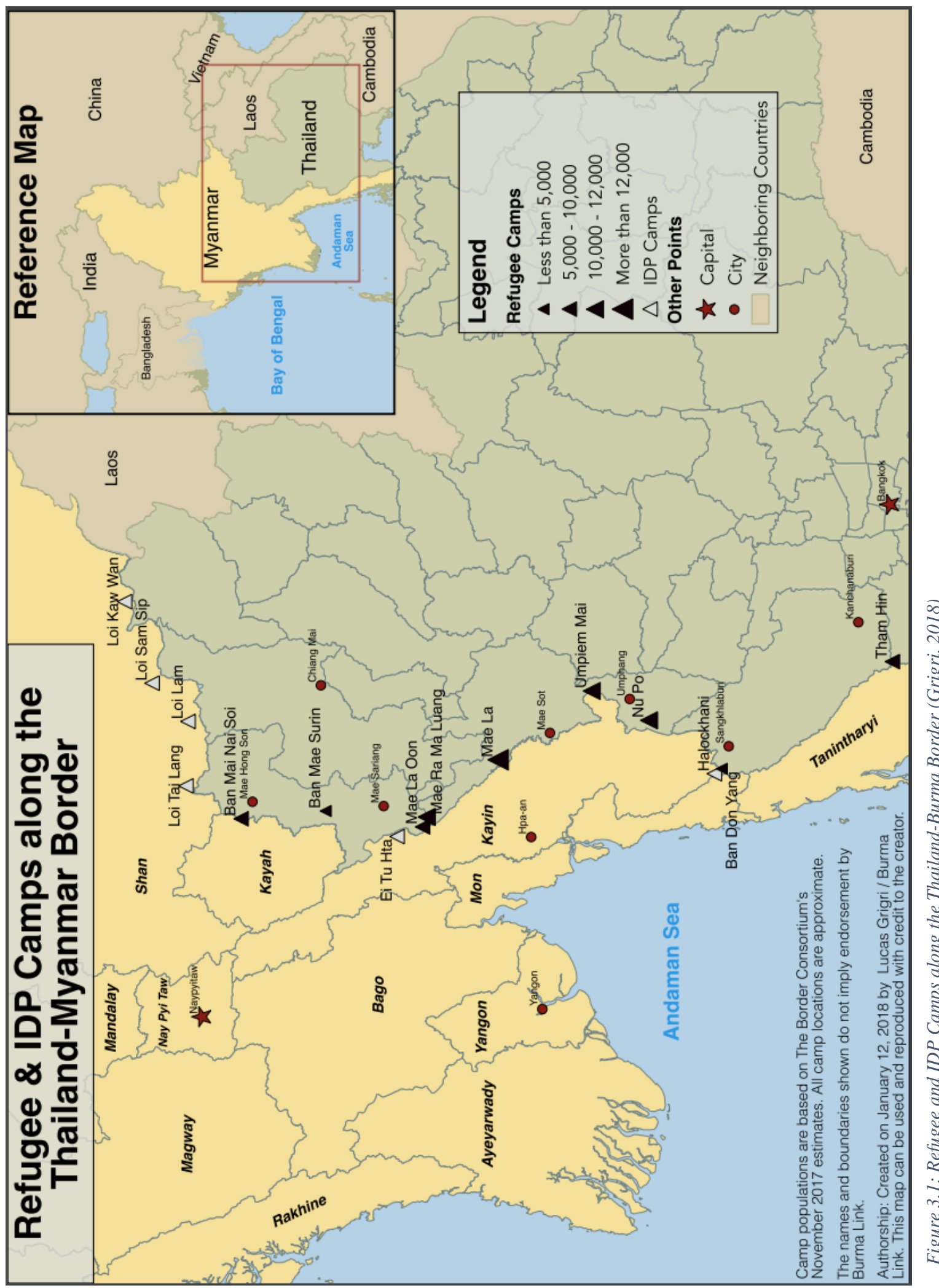

\subsubsection{Mae La Camp}

Mae La Refugee Camp was established in 1984 in Tak Province and is the largest refugee camp for people from Burma in Thailand, currently housing over 37,000 residents (The Border Consortium, 
2019). Because of its proximity to the ongoing civil conflict in the Karen state, the majority of the population of the camp are Karen. Small numbers come from Mon State, the Bago Region, Yangon city and the Ayeyawaddy region (The Border Consortium, 2019).

Figure 3.2 shows the demographic breakdown of the Mae La population. Noticeably, over one third of the population are of school age. This is because Mae La's large size has allowed a range of educational opportunities and it is now considered a centre of study for refugees. While most children have been born in the camp, the student population also includes a few thousand students from other camps or over the border in Burma who are registered as temporary inhabitants (The Border Consortium, 2019).

\begin{tabular}{|l|l|}
\hline \multicolumn{2}{|l|}{ Breakdown by age } \\
\hline$<5$ Yrs & $11.2 \%$ \\
\hline $5-7$ Yrs & $33.1 \%$ \\
\hline Adult & $55.6 \%$ \\
\hline
\end{tabular}

\begin{tabular}{|l|l|}
\hline \multicolumn{2}{|l|}{ Breakdown by gender } \\
\hline Female & $51.1 \%$ \\
\hline Male & $48.9 \%$ \\
\hline
\end{tabular}

\begin{tabular}{|l|l|}
\hline \multicolumn{2}{|l|}{ Breakdown by ethnicity } \\
\hline Karen & $84.8 \%$ \\
\hline Burman & $3.9 \%$ \\
\hline Other & $11.3 \%$ \\
\hline
\end{tabular}

Figure 3.2: Demographics of Mae La Camp (Statistics from The Border Consortium, 2019)

\section{"Static transience" in Mae La Camp}

When it was established in 1984, the refugee settlement of Mae La was a sprawling camp set among the Tak Province Hills. Its population of around 1,000 fled following the fall of the KNU, but the violence followed them across the border and a number of settlements in Thailand were raided. After these events, Thai authorities began to consolidate the settlements to improve security and Mae La was designated the principle refugee camp in the area in April 1995 (The Border Consortium, 2019).

Since then, resettlement numbers have been low and many families of refugees have been living in Mae La Camp for over 30 years (IOM, 2019). Life in the camp seems to be a permanent settlement. As well as schools and health centres, there are markets, stores and a barbershop that line the winding streets of the Mae La hills. Many people, especially those under the age of 30 who were born into the camp, see it as their home because it is the only place they know. Others, however, feel that this secure 'home' can be restricting:

Living in the camp is similar to living in prison because I can't go outside or make my own decision. I can commute only in the camp. The camp is surrounded by barbed wire. If we go outside of the camp, Thai police will arrest us. In the long run, it affects not only my physical but also my mental health. (Christine, 22, Karen refugee: Burma Link, 2015)

And yet, there is also an eerie liminality to Mae La Camp life - the sign outside the camp reads "Maela Temporary Shelter Area" - the name given by Thai authorities who can close down the border camps 
at any moment they choose. Since Burma transitioned to a civilian government, Thailand has given a stronger indication of their desire to close this and other refugee camps. Furthermore, NGO services in Mae La and other camps have reduced and many have moved on already. Their withdrawal has caused growing concerns for the refugee population as most have no village to go back to and many do not feel safe to return (Chandran, 2019). The lack of long-term sustainability in Mae La Camp has created a climate of fear and stress among refugees, and many feel that this, compounded with past trauma from conflict in Burma, has increased the occurrence of mental health issues for camp residents (Burma Link, 2015).

Rahul Chandrashekhar Oka (2014) calls this permanent/liminal life of a refugee camp, 'static transience' in which the confinement of a camp's borders combined with the vulnerability of a temporary home can decrease refugees' sense of safety. In order to overcome these insecurities, Oka suggests a routine of daily-life activities in which "normalcy and dignity become necessary conditions for resilience, adaptability, and survival." (p. 33).

\section{$\underline{\text { Livelihood programmes }}$}

Despite the uncertainty of camp life, programmes have been set up by NGOs and refugees themselves which "improve the psychosocial health of refugees by giving them productive activities to engage in." (Maclean, 2014, n.p.). There is a range of community activities and vocational training opportunities with the purpose of preparing refugees for income generation. Interventions include "organic farming, cooking, hairdressing, motorcycle repair, income generation through pig-raising and micro-credit for small shops, soapmaking and handicraft-making" (Maclean, 2014, n.p.). In my study of 'agency' in Chapter 6, I aim to understand how the Partners MHM programme affects the women of Mae La's access to activities like these.

\subsection{The Karen people}

\subsubsection{The Karen population is multiple and diverse $\mathrm{e}^{3}$}

There are limitations to identifying the Karen people primarily by a language or name; the Karen group is complex and fluid and instead shares a "cluster of traits" which differ slightly between individuals and family groups. While these include language, religion and social organisation, political and

\footnotetext{
${ }^{3}$ A disclaimer: As I write this section about Karen culture, I recognise that I am dealing with politics of representation. Bin Wu (2014) argues that for community-based research, local context knowledge should be generated by the stakeholders themselves. Therefore, as someone who is not from Karen background, I cannot speak comprehensively for their culture. Instead, the information in this section, obtained through secondary research, is an overview that offered background knowledge to my research. This knowledge is partial and disembedded but I have included it so that the reader can briefly understand the cultural background behind the research that follows.
} 
economic interests have been stressed as more integral to Karen identity than "ethnic" distinctions (Neiman, Soh, \& Sutan, 2008).

Among the Karen people, there are three main languages, S'ghaw Karen, Eastern Pwo Karen and Western Pwo Karen. There are several more dialects and accents that can make it hard for Karen people from different areas to understand each other. However, the most common language is S'ghaw Karen, which is used in schools and many community meetings in Mae La Camp (Neiman, Soh \& Sutan, 2008). There is also diversity in religion. 70\% of Karen are Buddhist, animist, or Buddhist-animist and around $20 \%$ to $30 \%$ are Christian (World Relief, 2018). On the other hand, there are common family values that are held by most, such as free choice of marriage partner and strict monogamy in marriage. Important values include humility, modesty and respect for elders (World Relief, 2008).

\subsubsection{Gender and reproductive health}

Traditionally, Karen culture is a matriarchal society. Lineage is traced through the female line and residence is matrilocal, a husband living with his wife's family after marriage. Within Karen animist beliefs, the head of a 'spirit clan' is a role that falls to women, so a husband becomes part of his wife's clan at marriage (Karen Organization of Minnesota, 2017). But this respected role does not necessarily mean women have equality and safety.

In pre-colonial Karen state, there was an expectation that men provided for their family by working in the fields. The women would clean, cook and collect water as well as working in the fields alongside men (Yang, 2018). Currently, the pressure to provide for the family's needs has been a point of stress for Karen families who have moved to refugee camps because opportunities for work and farming are low. The Karenni National Women's Organization estimated that in Karen camps, up to $60 \%$ of women had experienced some form of gender- based violence, many as a result of domestic fights in the stress of the camp environment (Maclean, 2014). Several reports also record extensive gender-based violence experienced by women at the hands of the Burmese military (Maclean, 2014).

In terms of women's health care, while most Karen are receptive to the idea of western-medical health care, many are hesitant to receive care that is culturally insensitive or unfamiliar. For example, a 2008 study (Neiman et al.) in a Karen refugee camp reported that 66 out of the 89 women who participated preferred to deliver their babies at home amongst close relationships, rather than in clinics or hospitals where male health staff could be present.

Information on refugee and migrant communities from Burma suggests that reproductive health education for Karen and other refugees from Burma can be low. Khin's study (2002) of the Karenni refugee population's knowledge and practices of adolescent reproductive health reported that shyness around the topic was a key barrier to young women's access to menstrual health education. The study 
revealed that many girls had a low or incomplete understanding of the menstrual cycle and that discomfort during periods was prevalent, particularly for those without sufficient sanitary materials.

On the other hand, women and girls from Burma have their own ways of understanding and dealing with menstruation. For example, there is a belief that those on their period should not eat spicy food, wash their hair or eat tea leaves. "If not followed, girls fear that their fertility will be damaged in some way," writes Bartholomew \& Calder (2018, p. 5). I would like to understand the specific beliefs and practices of the Karen women I interview and in which ways they consider these helpful for managing their periods.

\subsection{Institutional context}

Thai-Burma border camps are highly organised with refugees serving on 'Camp Committees' which are the management and administrative bodies of Mae La Camp. Elected camp leaders and committees represent refugees and are responsible for the the day-to-day coordination of the camp and camp services in collaboration with NGOs, UNHCR and local Thai officials and authorities (Neiman et al., 2008). For example, the Karen Refugee Committee - Education Entity (KRC-EE) is a committee that provides education, with the support of international NGOs such as World Education. Other organisations in the camp include the Karen Women's Organisation (KWO) which seeks to improve women's participation in all aspects of Mae La life and society (Burma Link, 2015).

As previously mentioned, international organisations have faced funding cuts and restrictions which make it hard for them to operate in the Thai border camps. The UNHCR, the United Nations Children's Fund (UNICEF) and the International Committee of the Red Cross (ICRC) struggle to provide protection and provision (Human Rights Watch, 2007). The Border Consortium, the main agency that provides food and shelter assistance to the refugees, has also had recent funding cuts which have caused them to cease provision of non-food items (The Border Consortium, 2016). Therefore, Partners' menstrual health assistance has become even more important in recent years.

\subsubsection{Partners Relief \& Development}

Partners Relief \& Development is an international organisation with the mission of promoting 'Free, full lives for children affected by conflict and oppression' (Partners, 2019a). Founded in 1996 by Steve and Oddny Gumaer, Partners began with an effort to help one orphaned girl in Sho Klo Refugee Camp in Thailand (Partners, 2019a). At first partnering with organisations like Youth With A Mission (YWAM) and Free Burma Rangers, the founders and first staff saw a need to "go where no-one else goes". Partners found that, as a small organisation, they could reach locations on the border and in Burma where large NGOs faced restrictions (D. Gibbons, personal communication, July 2, 2019). As it 
grew, it became Partners' goal to provide support for refugees, internally displaced people, stateless people and later migrants in Southeast Asia and the Middle East.

As its name suggests, Partners has two parts: relief and development work. Their Sustainable Development method of service includes healthcare provision and agricultural training, support of 'sustainable schools', income generation projects and education assistance. The Emergency Relief method includes short-term provision of food, shelter materials and other necessities during and soon after times of crisis (Partners, 2019b). The provision of reusable menstrual products under the Days for Girls programme straddles these two areas - mostly it is a relief method but the surrounding education about reproductive health has intended long-term community benefits.

\subsubsection{Days for Girls}

Founded in 2008, Days for Girls International (DfG) is a non-profit organisation with a vision to promote young women's dignity, health and opportunity through menstrual care and health education. Operating in over 100 countries, they believe that reproductive health awareness, education, advocacy and accessible feminine hygiene will mobilise girls and women and strengthen communities (Days for Girls, 2019a). The 2017 'mission and vision' reads: "Days for Girls increases access to menstrual care and education by developing global partnerships, cultivating social enterprises, mobilizing volunteers, and innovating sustainable solutions that shatter stigmas and limitations for women and girls" (DfG 2017 Annual Report, p. 3). The organisation's initial inventions focused around the distribution of disposable pads, but it was soon found that there were seldom adequate disposable facilities in the places of distribution. In response, Days for Girls began designing reusable, washable, long-lasting pads that would allow young women ongoing menstrual care (Days for Girls, 2019a).

As part of their operations, Days for Girls runs: a volunteer programme of sewists in the US, the UK, $\mathrm{NZ}$ and other countries who make 'DfG Kits'; an enterprise programme of individual micro-enterprises that employs low-income women to make the kits; and DfG Kit distributions to communities in its 'target countries'. While the organisation is an independent non-government organisation, it works in collaboration with other institutions at international, national and community levels (Days for Girls, 2018). In its target countries, Days for Girls occasionally works with local NGOs to aid delivery of its 'DfG Kits', as is the case in the Days for Girls-Partners partnership.

DfG Kit distribution has been widely and positively appraised. For example: "In countries in which the DfG program has been successfully implemented (e.g., Uganda, Ghana, Guyana), girls' attendance in secondary school has improved and the social stigma from unsanitary and unhealthy feminine hygiene habits has been reduced" (Kitch et al., 2017, p. 25).

Days for Girls has started to distribute to new kinds of communities - for example Partners' distributions to refugees and migrant communities in Southeast Asia. In order to address different needs 
in these environments, the organisation will benefit from quantitative and qualitative research. Gaining an understanding of washing facilities as well as community and cultural structures will increase the chance of safe and effective distribution in difficult environments.

\subsubsection{Partners' Menstrual Health Management programme}

In 2015, a woman connected to Days for Girls contacted Partners and asked if they would join with them to produce the reusable kits (Figure 3.3) and distribute them to the refugee and internally-displaced girls and women with whom they worked. Partners already had a workshop in the border-town of Mae Sot where they ran a sewing training programme for migrant women, and they agreed to take on the project (Tendero, 2019). Over the last few years, the programme has developed and kits have been distributed to women in Shan State, Kachin State and Karen State in Burma, as well as to the Mae La border camp and migrant communities on the border in Mae Sot. Sewists in the workshop in Mae Sot continue to make the DfG Kits, alongside other products (Tendero, 2019).

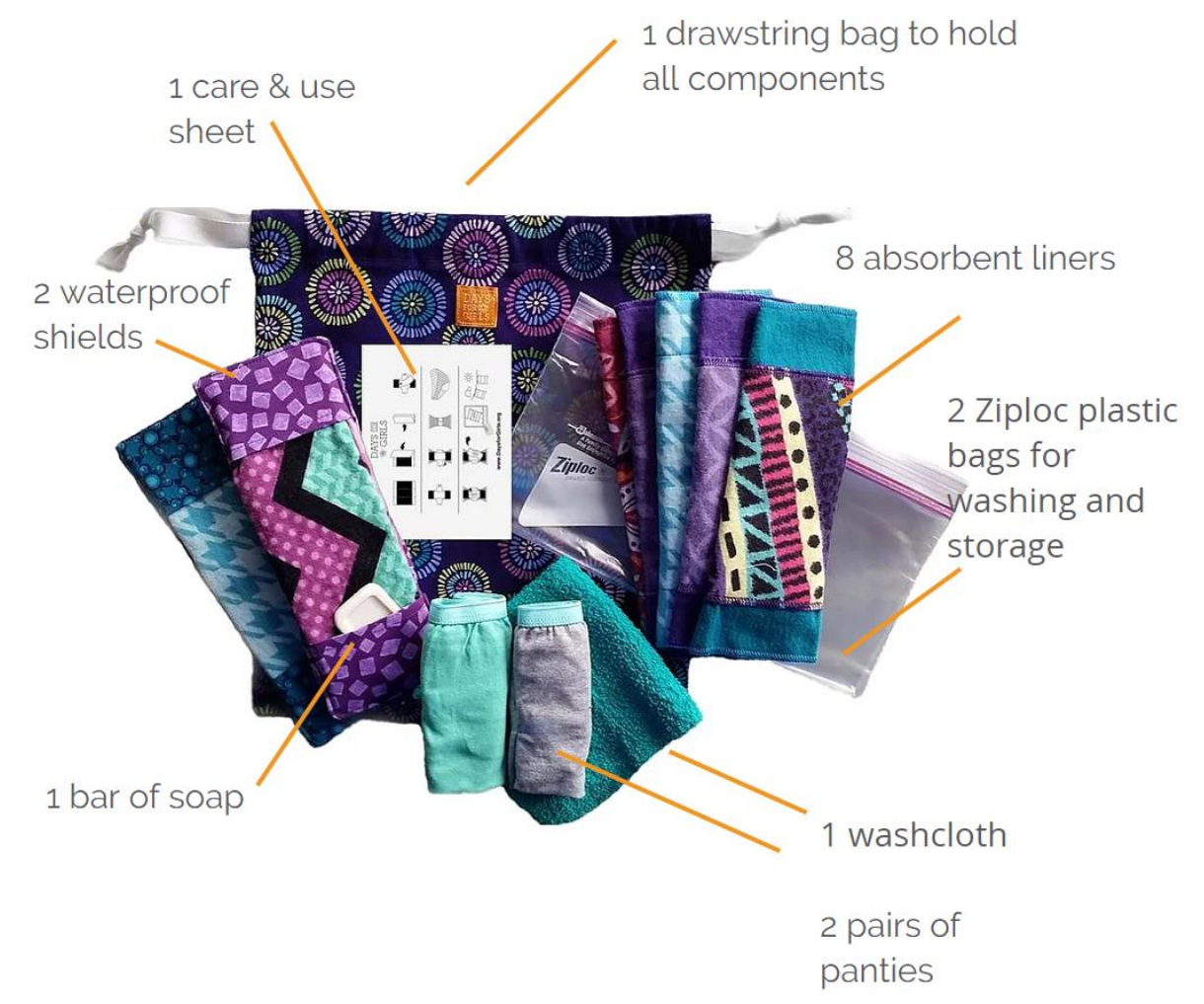

Figure 3.3: The Days for Girls Kit (Days for Girls, 2019)

The 'DfG Kit' typically includes underwear, soap, reusable pads, a washcloth and zip-lock bags. The pads consist of waterproof shields that lock around the underwear, and separate absorbent liners so the absorbency can be modified for flow (by stacking one or multiple liners). The design practicality is explained by Days for Girls (2019b, n.p.): 
The bright colors camouflage staining. The absorbent liners unfold to look like a washcloth, which allows women to wash and dry them outside in the sun without causing embarrassment. All of these design choices add up to a lasting, easy-to-care-for solution.

As a relatively small NGO, Partners (at the time of my research) had not yet had the funding to carry out a full evaluation of their menstrual health programme. However, they were interested in understanding what changes they could put in place to improve the programme that could make women more comfortable and their training more appropriate. Partners also wanted evidence for the difference the programme was making. They had already heard positive stories through word of mouth, but they were hoping to understand what specific changes the menstrual health programme has made to women's lives. Within the aims of this research, these two knowledge-gaps are addressed, especially in relation to women's confidence and agency (see Chapter 6).

\subsection{Conclusion}

This chapter has provided insight into the 'geopolitical context' of this research project. It reflected on the constantly evolving settings of refugees and migrants in Burma and Thailand. Burma has a deep and complex history of conflict that continues to persecute ethnic minorities. This has pushed many to seek life in Thailand as refugees and migrants, but this life often involves a 'static transience' such as described in the border camp, Mae La. I have suggested that Karen women's relationship with health and menstrual health is shaped by their cultural beliefs and experiences of displacement (Karen women make up most of this study). Finally, I have described the way that Partners' menstrual health programme is situated within and affected two institutions - Partners Relief \& Development and Days for Girls International.

This setting is the context for the stories of the women who will be represented in the following chapters. The women in question live complex lives, each differently affected by and affecting life in their refugee and migrant contexts. But they are each connected by experiences of displacement, the pursuit of life in a new country and the need to navigate NGO relationships through this process. I will explore how the Partners menstrual health programme addresses, considers and learns from women's refugee, migrant and menstrual health experiences, particularly in Chapter 8. 


\section{Chapter 4 - Research Approach}

\subsection{Introduction}

The methodological journey for this research project has been varied and evolving. While I had an epistemology and a rough methodological plan for my field work before I left, I decided not to pre-plan my methods in detail. This was partly in keeping with feminist ideas of inclusiveness and Appreciative Inquiry ideas of collaboration (Cooperider, 2009; Reinharz, 1992). Therefore, as I was looking to work closely and respectfully with Partners, I wanted to develop my research plans in communication and cooperation with them. This decision was also practical, as it was difficult to communicate about plans to those in Thailand while I was in New Zealand and didn't yet fully understand the research context.

This chapter provides an overview of my research approach - how I planned it, how those plans were carried out and how they changed. I begin with a discussion of my epistemology, followed by a description of and rationale for my methodology. I then discuss my research design and methods and how these align with both feminist and Appreciative Inquiry based frameworks.

Towards the end of the chapter, I present my reflections on ethical issues and limitations.

\subsection{Epistemology}

My 'ways of knowing' that have directed this research project cannot be found within one epistemology, but are shaped by my personal history, academic journey, religious beliefs and relationships. However, I acknowledge the benefits of "a lens through which a researcher looks at the world," where an established paradigm gives a purposeful direction and way of analysing which can help to deepen research (Kivunja \& Kuyini, 2017, p. 26).

Therefore, I chose to work within a feminist epistemology. I have used this approach to create my core questions and to question myself, as I progressed along my research journey. At its centre, I see a feminist epistemology as valuing women's voices and experiences, challenging ways of thinking about gender, while also being attentive to issues of power, ethics and knowledge creation (Hesse-Biber \& Piatelli, 2007; Rogers, Castree \& Kitchin, 2013).

Feminist epistemology grew out of a qualitative shift in the social sciences from the critique of the invisibility of women as subjects and researchers, to the critique of the methods and purposes of social science itself. This shift influenced the movement from WID (Women in Development) to the more structural WAD (Women and Development) thinking - where feminist research began to focus on research for women, not research about women (Gorelick, 1991; Rathgeber, 1990). 
Today, feminist scholars engage with gender far more broadly (as seen in GAD or Gender and Development) thinking. Rogers, Castree \& Kitchen (2013) summarise feminist epistemology as "an approach to understanding the world that emphasizes how gender influences the ways we come to comprehend and research reality." There is a strong critique of the traditional rules of research - that it should be objective, neutral and value-free - because of their embedded "unconscious patriarchal bias" (O’Leary, 2017, p. 156). A feminist approach acknowledges that the researcher is not neutral. Therefore, I am reflective of my own subjectivities and biases throughout this chapter. Throughout my research process, too, I tried to remain aware of how my personal understandings shaped my actions (tracked through a field diary) and writing (Jackson, 2006).

As well as a commitment to the empowerment of women, I have aimed to adopt other feminist values regarding power and knowledge creation. Taking on a feminist research epistemology necessitated that I acknowledge the power and position of the researcher and attempt to lessen this distinction (O'Leary, 2017). It meant valuing local knowledge, searching for subjective and diverse understandings and focusing on marginalised voices (Reinharz, 1992). I was encouraged to think about how I could engage with participants respectfully (to be both culturally and ethically appropriate) and ultimately to strive to do research that brings about positive change and contributes meaningfully to gender justice (Howitt \& Stevens, 2010; McEwan, 2001).

Increasingly, feminist researchers have begun to recognise that gender lines are only part of a wider web of lines of inequality and should not be analysed on their own (Kupiecki, 2015). In intersectional feminism, feminists engage with issues of difference between and across class, race, sexuality and other markers, "in an attempt to explain the interrelationship of multiple forms of oppression" (McEwan, 2001, p. 98).

Intersectional feminist researchers ask: "How does the inequality [women] experience differ when they are white or black, hetero- or homo-sexual, rich or poor?" (Kupiecki, 2015, p. 108). In my own research, I have attempted to analyse my findings with "complexity and variation" in mind - acknowledging the impact of race, income and other markers of difference such as 'position in an organisation' on participants' experiences.

Before conducting my research, I also wanted to gain an understanding of how feminist and genderrelated studies are seen by people from Burma. Acknowledging that this will never be unanimous, I still wanted to situate my lens and judge its relevance. Among my reading, I found some viewpoints that posit gender equality as a Western concept (The Gender Equality Network, 2015). They criticise Western feminist researchers for disregarding cultural systems in Burma: for example that women already have a 'high status' in society and that Western individualist values of freedom do not line up with their own emphasis on collective welfare. One response read: "It is not inequality, this is about our 
culture. This is our religious practice or this is our social practice.” (The Gender Equality Network, 2015, p. 17).

I wanted to reflect on this issue before I began my research. It is true that I had very little understanding of wider Karen or Burma culture, despite my contextual reading. So I read further. And I found a strong emergence of critique by women from Burma who do feel that their room to move in society is narrowed by cultural and societal norms (Khin, 2002; Nyein, 2019). This feeling was also expressed to me by some of the Karen women I interacted with in Thailand. I am limited by my outsider status in my ability to deeply analyse the gendered dynamics I saw in my research. Consequently, my intention with this research is to share the understandings of my participants and those closer to the context.

\subsection{Methodology}

As explored above, a feminist approach not only focuses on research outcomes - the process of conducting research is equally, if not more, important (Campbell \& Wasco, 2000). At the start of my planning process, I looked for a research approach that would respect all participants' stories and experiences. In previous research with people from refugee background, I found value in a strengthsbased methodology. I found it useful to counter the existing discourse around refugee issues which tends to take a 'needs' or 'burden' approach that "constructs refugees as passive recipients of welfare rather than acknowledging their resources" (Butler, 2005, p. 148).

Appreciative Inquiry - a practical, strengths-based approach often used in organisational development fit this purpose well. In line with feminist epistemology, it emphasises collaborative research as a form of social construction in action that brings about positive change (Reed, 2007).

While commonly used as a positive organizational development practice, Appreciative Inquiry was originally envisioned as a "social science of possibilities" (Cooperrider, 2013, p. 10). At its heart, "AI [Appreciative Inquiry] is about the search for the best in people, their organizations, and the strengthsfilled, opportunity-rich world around them" (Stavros, Godwin \& Cooperider, 2015, p. 97). It asks researchers to see the assets and 'wholeness' of their research subject and "inquire into that system's strengths, possibilities, and successes." (Stavros et al., 2015, p. 97). To do this, Appreciative Inquiry uses four processes (from Reed, 2007):

Discovery: "Appreciating what gives life" - a process of inquiry (usually interviewing) where participants are asked about what works well in a programme.

Dreaming: "Envisioning what might be" - encouraging participants to think creatively about possibilities for the future - without restrictions of resources or relationships. 
Designing: "Determining what will be" - confidently crafting plans for the future based on the Dreaming phase.

Delivery: "Planning what will be" - action planning, with more detail and often based on past successes and achievements.

For the purposes of my research, I have focused solely on the first two phases of Appreciative Inquiry. It was not my place as a researcher to make plans for the organisation. Only to ask questions and share evidence and experiences that may help the programme to follow their own 'designing' and 'delivery' in the future if they chose to do so.

I tried to be influenced by the following Appreciative Inquiry principles and assumptions from Reed (2007):

- In every society, organisation, or group, something works.

Appreciative Inquiry starts by focusing on the positive aspects of a programme because asking positive questions means "people naturally turn toward ideas and images that provide nourishment and energy" (Reed, 2007, p. 27). This allows deeper engagement with the participants and the building of a positive researcher-participant relationship. The principle doesn't imply that the problems people experience aren't real. But rather the aim is to create an environment with increased freedom from censure and blaming. "Problems and weaknesses are often much easier to address when evaluation takes an appreciative stance" (McNamee, 2003, p. 27).

- Appreciative Inquiry is anticipatory.

This is the idea that "the way people think about the future will shape the way they move toward the future" (Reed, 2007, p. 27). In my questioning, I have tried to ask participants about their different imagined futures, rather than the negative aspects of their present. Their ideas for change in a future "full of possibility" both gave me insights into their current experiences and helped to give a constructive feel to many of my interviews.

\subsubsection{Personal motivations for my choice of methodology}

Because I have family that works at Partners Relief \& Development, I was in a unique situation of motivation to maintain a good relationship with the NGO. I am aware that sometimes researchers' critique can cause tension between themselves and the organisations with whom they work (Clark, 2009). I felt that using Appreciative Inquiry, I could take part in positive "active research collaboration" which according to Hinkin, Holtom \& Klag (2007), "is the key to producing research that is both relevant to and respected by both parties" (p. 112). By using a strengths-based and 'appreciative' methodology, I felt more comfortable that my research would be respectful of the work of participants. 
During my fieldwork, I was thankful for this decision. There were several times when I had to acknowledge how accommodating and helpful Partners staff were of my research. They set up interview locations for me, drove me hours to Mae La Camp, helped with my administration and were kind and helpful at every turn. I was also impacted by the selflessness of staff - I could see clearly that they go above and beyond for the communities where they work. While the organisation wanted to know about aspects it could improve, I felt that going into the process with a solely critical lens would not have done justice to the kindness I witnessed. In this research project, I do implement significant feminist and development critique. But I have tried to set this in a future-focused and strengths-building frame, as recommended in Appreciative Inquiry.

\subsubsection{Another methodological influence: Follow the Thing}

'Follow the Thing' is a methodology developed by Ian Cook that travels along the locations of a commodity chain of a product to hear the "human or other stories that are actually in those things" (Cook, 2016, n.p.). It is a style of research where 'things' are analysed as "ways of connecting together the lives of people who don't know anything about each other, but whose lives are intimately connected on a day-by-day basis" (Cook, 2016, n.p.). In this way, researchers can create a "fuller story" or ethnography of a process and understand its human effects (Cook, 2016, n.p.). At each site that the 'thing' (Days for Girls Period Kit) travels through (production, distribution and use), we can ask questions about the individuals as well as the cultural and structural contexts that shape and are embedded in the product.

While Follow the Thing has historically been used to raise awareness of ethical consumption, I thought its idea of connecting people and people's stories along a product chain could be useful for taking a more holistic view of Partners' MHM processes. In more recent literature, Cook \& Tolia-Kelly (2010) urged 'Follow the Thing' scholars to investigate the paths of materials that don't have a traditional 'consumption' story. For example, the MHM products I followed are re-useable, and are largely donated rather than purchased items. By instead following the path of a humanitarian relief resource, I questioned (in Chapters 5 and 6) how an NGO-run product-chain impacts the lives of the receivers, as well as the producers (particularly their confidence and agency).

This methodology was a peripheral, rather than a core part of my research process, but I used its orientation as I developed my interview questions, intending to appreciate the social relations along a chain of MHM relief. Follow the Thing has also influenced my analysis and presentation of results, as seen in Chapter 5 where I structure my findings according to the spaces along the MHM kit chain of production. 


\subsection{Research Design}

This research project evolved through collaboration with Partners Relief \& Development. Following feminist and Appreciative Inquiry practice, it would have been ideal to work on research planning with Partners and within the research context from the outset (Cooperider, 2013; McEwan, 2001). However, I was limited by geographical and time constraints and could only begin proper collaboration after I had a wide outline of my research. I was fortunate to have visited Thailand twice before and to have had family working near to my research topic who could inform my initial understandings.

In light of my epistemological approach, principles of Appreciative Inquiry and the orientation of 'Follow the Thing', I adopted a mixed methods methodology. Schoonenboom \& Johnson (2017) define the mixed methods approach as research that combines elements of qualitative and quantitative methods for the broad purposes of breadth, depth of understanding and corroboration. The approach allowed me to appreciate the depth of the programme's impact through qualitative analysis, to understand women's stories and experiences through qualitative interviews and to tailor research methods to each 'Follow the Thing' location.

The main methods of inquiry were surveys and semi-structured interviews. While feminist theory encourages qualitative inquiry, I found that a mixed-methods approach could contribute effectively to Partners' work and was helpful to my own understandings. I chose to use survey data as I had been told that quantitative statistics would be practically helpful for Partners as they applied for funding. At the same time, for my own research purposes and philosophies I valued the qualitative, in-depth personal understandings I gained from interviews and observation. I was able to use data from the surveys to help generate questions, and I saw that it added richness and strength to the quotes of participants. Because I had limited time to interview everyone involved in the programme, the quantitative data added breadth to the study and may have gone some way in making it more representative (O'Leary, 2017).

\subsubsection{Collaborating with Partners in the field}

I spent ten weeks in Thailand over the course of my research. I chose to base myself in Chiang Mai, where the main Partners Southeast Asia office is and where my parents live. I spent the first week going into the office each day with the goal to get to know the organisation better and begin to build relationships. I attended meetings, established my position as a 'Volunteer and Researcher' at the organisation and received inductions. I was also asked if I would sit in on a meeting about Partners' data collection and to help to edit their magazine. I was pleased to be involved in these activities as they gave me a much deeper insight into the organisation's values and the way staff work than I would have got from reading. Volunteering in this way also showed staff my motivation to be of service to them and I believe it helped build trust. I wanted to show that I valued reciprocity in our relationship - a principle valued in feminist research where "research is a two-way process and that researchers should not simply serve their own needs" (Hill, 2019, p. 58). 
My research took a somewhat phased approach, where initial and 'key informant' information was found that shaped the rest of the research process (O'Leary, 2017). An important initial phase was a 'pilot week' in Mae Sot, my main research location, where I conducted one survey and some informant interviews. This was helpful because it helped me to understand what was feasible within my research timeline and what sorts of approaches worked and didn't work. I also got to know one of my key informants over that week - Naw Thay ${ }^{4}$. Naw Thay has worked closely with the programme coordinator on the Days for Girls programme and while the coordinator was away, she was extremely valuable to my understanding of the programme. She also acted as my translator for many of the interviews. After each interview and the survey in the pilot week, I talked with Naw Thay in-depth about how it went and what could be changed about the way I worked. This reflective process was essential to my process of adapting a vague research outline into a workable, informed research plan.

Interviews with migrants and students in Mae La camp were organised in large part by the Partners staff who worked with these individuals. While I carried out proceedings, I was very reliant on these staff members because I was unable to communicate with boarding homes and workplaces to set up meetings without translation. While this was partly a positive thing, as it made the research collaborative, I was also aware of the gatekeeping issues that come with giving much control to an organisation whose programme is being studied. Defined as "those individuals in an organisation that have the power to grant or withhold access to people or situations for the purposes of research" (Burgess, 1991, p. 48), gatekeepers have the potential to limit the legitimacy of research results. My reliance on Partners was an aspect of my research that I couldn't easily change as it was a consequence of my choice to do research in an unfamiliar research context. However, Quinn (2014) argues that a mixed-methods methodology can limit possible biases. I believe that my use of multiple methods has helped to overcome the limitations associated with any one method.

\subsubsection{Living in the field}

While I didn't live in migrant communities for the entire research process, spending months living and working in Thailand enriched my research abilities. I tried to take a reflexive and critically engaged approach to my day-to-day life in Thailand as I engaged with expats, Thai national citizens and a wide range of migrant communities from Burma. While my research was mostly based in Mae Sot, I felt that in fact it was "multi-sited" in that my personal and research-related roles and relationships converged throughout my time in both Mae Sot and Chiang Mai (Cupples \& Kindon, 2003).

By becoming part of Partners' 'team', I was able to be present for a number of events that made me feel embedded in the organisation - from celebrations to farewells. Notably, I took part in Partners' yearly week-long Strategic Planning Sessions. While I had predicted I would only be invited to the general sessions of this week, I was instead placed in the 'Livelihoods' department (the part of Partners where

\footnotetext{
${ }^{4}$ Pseudonym. I have used pseudonyms for some participants for anonymity (see 4.5.5 'Representation').
} 
the Days for Girls programme currently sits) and asked to contribute alongside other staff. Throughout the week I heard stories from across the organisation, took part in prayer and worship sessions and generally was made to feel included and valued. While I cannot claim to have the perspective of someone who lives in Partners' context, these experiences allowed me to better understand what participants saw in the organisation and how they themselves looked out on the world.

Throughout my time in Thailand, I kept a fieldwork diary to encourage "reflexivity and iterative practices" (Hall, 2019, p. 77). Feminist geographers encourage researchers to practice reflexivity throughout their work, rather than retrospectively (Sultana, 2007). In this diary, I noted my observations, my growing and lessening biases, and how I saw aspects of power and positionality in relation to participants. I also used this space to reflect on each stage of my research process. I would note problems and questions, and through "writing by processing", would often end up with a list of 'next steps' to guide my actions. This more careful and iterative cycle is thought to be invaluable to research, especially in projects about sensitive topics where ethical questions require careful thought (Browne, 2013). In my analysis and writing, I was also able to draw key themes from these reflections.

\subsubsection{Quantitative surveys}

The quantitative aspect of this research project took the form of four surveys of students in Mae La boarding homes who had received the menstrual hygiene kits. Days for Girls International, the organisation that designed the kits, has developed an existing international system of evaluation where girls who have received the pads complete an anonymous survey in a classroom (Days for Girls, 2018). Students are asked to close their eyes for the duration of the survey and with the help of a translator, they are asked about their experiences with MHM and asked to use hand signals to answer yes/no questions. This method allows evaluators to survey groups with a range of literacy levels, and to conduct anonymous research in an environment familiar to the student participants. Anonymous voting is also recommended by the Toolkit for integrating menstrual hygiene management (MHM) into humanitarian response by Sommer, Schmitt \& Clatworthy (2017) because it is more sensitive of those who may feel uncomfortable with expressing themselves on the topic of menstruation.

For these surveys, I recruited participants through the boarding homes and with the help of MHM programme staff at Partners Relief \& Development, who had distributed pads and taught about MHM to the girls already. The caregivers brought together all their current students who had received Days for Girls Kits (excluding those that had since left school), so I estimate that I surveyed between 70-80\% of the total affected population. This recruitment method was also beneficial because it allowed girls from a wide age-range to be part of the research - for example thirteen or fourteen-year-old girls who may have just started menstruation may have had different menstruation experiences to older students. During the surveys, the caregivers and/or known members of Partners staff were present to provide familiarity and possible comfort to the participants. 
I had intended this data to be useful not only for Partners, but for Days for Girls International's wider evaluation programme. But interestingly, soon after returning to New Zealand, I was informed by the organisations' evaluation team that they had decided not to publicise survey results as they felt the data collected was unreliable. On reflection, I can imagine a few reasons for this. Apart from possible mistranslations or students opening their eyes and changing their answers after seeing what their friends were saying, there could have been another cultural issue at play. I noticed that many more students were making the hand-signal for 'I prefer not to answer' than 'no'. I wondered if this was affected by a Southeast Asian preference that I had been told about earlier - that many people are uncomfortable saying 'no' as part of "saving face" (Kemp, 2009). While this could entirely be a stereotype, it is possible that the data was therefore skewed towards 'yes' answers, and I have kept this in mind as I analysed survey results.

\subsubsection{Semi-structured interviews}

Consistent with the acceptance of multiple and diverse views in feminist epistemology, semi-structured interviews included broad, open-ended questions (see Appendix) to encourage participants to share their perspectives as openly as possible (Creswell, 2014). Interviews were audio-recorded and took place in a variety of locations. I spoke to Partners staff, migrant women - including those who worked sewing Days for Girls Kits, and students in Mae La Refugee Camp. While I attempted to learn from a set of experiences as diverse as possible, the samples chosen are not meant to be representative. Instead I aimed for the interviews to follow the qualitative research approach which focuses on the knowledge and lived experiences of a few (Creswell, 2014) in order to understand more in-depth perspectives.

Following Appreciative Inquiry, all interviews were designed with questions that would firstly "evoke a real personal experience and narrative story that helps the participant to identify and draw on their best learning from the past", and secondly go "beyond the past to envision the best possibilities of the future" (Reed, 2007, p. 36). In this 'Dreaming' phase, I asked participants to dream beyond the programme offered and to include their own traditional previous knowledge of MHM in their dreaming.

Interviews were conducted in participants workplaces and schools/boarding homes. While most of these locations proved appropriate (quiet, enough privacy but not too secluded), some made me aware of geographies of power within a room. For example, at the first boarding home I visited for a survey and interviews, I was greeted by an assembly of the whole boarding home and was asked to stand on stage and address the school. As I fumbled my way through this, I was aware of the very uneven power relationship that was being established between the students and myself - that I was a guest of honour. I thought this may make it difficult for students to be open with me about their experiences with menstruation, especially in the one-on-one interviews I had planned. In order to mitigate this dynamic, I asked the students I interviewed to sit on the floor of the stage with me as we talked. The two students who volunteered seemed to want to do the interview together, and as we talked I could see that their 
conversation bounced off each other in a comfortable style. While I hope this helped to bring a more equal feel to the meeting (Chambers, 1997), I could not truly subvert the power structures involved in this and other interviews (Kindon, 2012). I could not escape how I may have been positioned due to my ethnicity, class and the power and authority I had already exerted as a foreign facilitator of this project.

\subsubsection{Mapping}

In an attempt to overcome some barriers of language, I chose a visual 'mapping' method to be part of my interviews with students who had received Days for Girls Kits. In order to map how accessibility and mobility affects students' menstruation and use of the Days for Girls Kits, I asked them to draw maps of their neighbourhoods and use a coding system to mark where they could and couldn't go during their period (See Figures 6.4, 6.5, 6.6).

This type of visual research has not been widely used in MHM studies, I believe it can be a valuable approach for presenting participants' (in this case, women's) voices. Barron, Mitchel \& Yule (2017) argue that such methods can be helpful conversation aids, especially when talking with young people about sensitive topics. Indeed, I found that most students engaged well with the visual mapping method and often answered questions in relation to the maps they had drawn. The maps on their own also produced helpful references for my analysis of the effects of the MHM programme.

On the other hand, visual methods cannot fully bridge the cultural and power gap between facilitator and participants (Cornwall, 2004; Kindon, 2012). While the activity required only a low level of literacy, it also relied on the physical abilities of drawing. While I emphasised that the items on the map didn't need to be drawn well (or that they could use 'boxes' for buildings), I still sensed a hesitancy from some of the participants about having to draw in front of people. As they pushed through their discomfort and followed my instructions, I became aware of the embedded power I had, even in what I thought would be a casual, 'fun' activity.

\subsubsection{Research collation and analysis}

An iterative process was used in the collation and analysis of the findings from this research process, as encouraged by Barbara \& Benjamin (2006) for its ability to generate an "emerging understanding" (317). Firstly, carried out a rudimentary coding process alongside a data analysis from the surveys to produce an initial evaluation about the Partners MHM programme. After receiving feedback on this report, I turned to my main phase of analysis.

In line with feminist epistemology, I wanted to begin my main analysis with an inductive process where themes would 'emerge' from participants' ideas, rather than demarcating codes and categories that I would assume their words to fit within (Leurs, 2017). Thus, I began by reading through each transcript with an open mind and trying to pick out themes that seemed important to the participants - through their expressions, repetitions and omissions. 
Next, I began a more deductive process. I combined these themes with other questions that emerged from my secondary research into menstrual health and NGO practice as well as my methods of Appreciative Inquiry and Follow the Thing to eventually sort the research findings into chapters. I used NVivo, a qualitative data analysis software especially designed for text-rich data to further code transcripts. NVivo allowed me to identify connections and group concepts across several themes, which helped me to understand the interconnectivity of ideas (Cope, 2010). I used hand-written mind maps and lists alongside this process, where I felt that the 'demarcated box' aspect of NVivo was limiting.

I then combined photographs, maps, tables and graphs of quantitative data as well as my themecategorised quotes to create the chapters of my thesis analysis. As I wrote, I continued re-formulating research data, continued to read concurrent studies and had further conversations with those I had met in Thailand that further shaped my research.

As I am not a refugee from Burma, or even an invested Partners staff member, my analysis is not grounded in a deeply-embedded understanding of Karen or other cultures from Burma. Instead, I have offered a perspective from informed by my reading of MHM literature, and a synthesis of a diverse range conversations I had with participants. As I wrote, I tried to be aware of feminist epistemology that "make[s] us more aware of how knowledge is partial, situated and contextual" (Leurs, 2017, p. 115). In particular, I attempted to acknowledge complexity and variation between experiences especially when related to power and knowledge, as encouraged in intersectional feminism (see Chapter 2). I tried to keep in mind the differences between participants' roles, ethnicities and classes and how these may affect their menstrual experiences, as well as my positionality and how that may affect my analysis.

\subsection{Ethics and reflections}

\subsubsection{Ethical process}

Before travelling to Thailand, I received ethics approval from Victoria University of Wellington Human Ethics Committee, and research approval from the Thai Research Council. Clauses included: all participants would have pseudonyms or other non-identifying references, participants above the age of 16 would be interviewed where possible, participants would have the opportunity to review and change their transcripts if they wished to and that I would only carry out research in locations approved by the Thai Research Council. Other than these considerations, the ethical framework of this research was guided by Feminist and Appreciative Inquiry principles, as detailed in this chapter and in Chapter 1.

\subsubsection{Reflection on feminist epistemology: About vs for}

A critique of feminist approaches to development research concerns the "valuing the marginalised voice" discourse. Gorelick (1991) argues that feminist research rarely uncovers all oppression; there is 
always a level of subconscious internalised oppression that trying to 'give women voice' doesn't address.

In reality, my research was perhaps just as much 'about' women from Burma as it was 'for' them. Despite clear goals to do helpful research with these participants at the centre, I was part of, and helped to perpetuate, typical power structures of research. The beginning conversations where this study was suggested were between Partners staff and myself - not initiated by the women who received Days for Girls Kits. While Partners' enthusiasm likely arose from feedback and questions by these women, I cannot claim that this was a bottom-up research project. It had not been decided upon or devised collectively as ideally suggested in research that aims to most deeply involve communities (Tuck \& Fine, 2007).

My use of academic theory, and especially the abstract concepts I take from Feminist theory, also pose a problem if I intend my research to be entirely 'for' my participants. I have struggled with the level of accessibility of the concepts and language I use in this study. Many of my participants (because of language barriers, unfamiliarity with the academic theories or just time constraints) will not be able to read my thesis, even some of those running the MHM programme. Merrifield (1993) writes that communities with whom researchers work often don't have access to academic literature which is often filled with jargon and other inaccessible themes for non-specialists.

As an attempt to mitigate this issue, I produced a report for Partners that was practically, rather than academically based. I received positive feedback on this report and was told by Partners staff that it will help them with their relief work and help with finding applications for the programme in the future. I have to acknowledge that for the purposes of my thesis, I am writing both about and for the programme and about and for the women who are part of it.

\subsubsection{Appreciative Inquiry: Limitations, reflections}

As my research was for the large part, researcher-determined, I acknowledge that my research did not fully follow the spirit of Appreciative Inquiry. The literature on the methodology mostly rejects researcher-determined design. Research is encouraged that is not only collaborative, but led by stakeholders and participants (Cooperider, 2013; Reed, 2007). While my research was led in part by Partners Relief \& Development, the scope of a Masters research project and the limited time I had in Thailand meant I could not carry out a fully participatory research project, but I tried to act in a collaborative spirit.

Another point of reflection for me has been the appropriateness of Appreciative Inquiry in the Karen cultural context. While the questions were aimed towards positive aspects of the programme, there is an implication that the important struggles can also be uncovered. Through this process, the focus toward growth, "generative questions" and "generous listening" is meant to encourage an environment where 
difficulties can be discussed (Nicholson \& Barnes, 2012, p. 7). However, in my research, this did not often naturally happen with those from communities from Burma. Whenever I asked questions towards 'growth' or improvement in the future, I sensed a discomfort from participants who benefited from the programme and many did not have an answer. Through several discussions, I learnt that "not defaming others" or grangai (Thai) is a deeply embedded part of cultures within Southeast-Asia and that these participants would have been uneasy if anything they said came across as criticism of Partners. In a similar way, Collie et al., (2009) reported that in her participatory research with young Assyrian women, there was discomfort when participants were asked to share anything that might hinder the reputation of their family members or community elders. While I thought that Appreciative Inquiry would avoid his type of discomfort, in reality, the 'future-improvement' focused questions could still be thorny. This issue is discussed in Chapters 6 and 8.

Overall, I received positive responses to this way of carrying out research from participants and stakeholders. I cannot claim that the research design was the sole reason for these smooth interactions, but I am happy with the relationship-building aspects of Appreciative Inquiry and the mostly-positive conversations it encouraged.

Finally, I am aware that my motivation to use Appreciative Inquiry reveals the partiality I have as a researcher - I did not want to use an overly critical or 'tearing down' approach that may jeopardise my relationship with staff members at Partners. In my analysis, I have tried to recognise the limitations that this brings; I may not have asked the most hard-hitting questions, and I may have missed out on uncovering some of the weaknesses in the programme. But I also believe there were benefits to my partiality - as discussed in Chapter 1.4.1 "Embeddedness and Objectivity", my relational embeddedness encouraged me to research carefully and sensitively.

\subsubsection{Translation}

A significant limitation to my research was the language barrier between the Karen and other-language speakers I interviewed and myself. I had not learnt the language of many of my research participants partly because they represented several different languages, and partly because I did not prioritise this as part of my research. Therefore, translation was an integral part of this research project that caused many ethical questions. Despite having confidentiality agreement from my translators, I was aware that having a third presence in interviews also meant further ethical issues, as "[t]ranslation is always interpretative, critical and partial”. (Haraway, 1991, p. 195).

This posed an issue of representation. While I cannot claim to truly represent participants in my writing (Alldred, 1998), translation in interviews adds another layer of interpretation of participants' voices as discussed in Chapter 6. One of my key translators was Naw Thay, who worked in the Days for Girls programme at Partners. While her attendance was practically very helpful, I also knew that she could not be an unbiased presence. In order to mitigate any impartial effects, I chose to use a different translator 
for interviews with the sewing staff (who are employed by Partners) and I had this translator listen to a recording of one of our student-interviews to verify and cross-check interpretations.

\subsubsection{Representation}

This research process has involved further issues of representation. As mentioned, this research has not achieved a fully participatory practice of "writing with" (rather than "writing about") participants (Hill, 2019, p. 21), but I have tried to be mindful of how I have framed each participants' lived experiences.

In terms of representation though naming, I have had to negotiate how to maintain participants' anonymity (as I promised them before they agreed to speak with me) without making them "faceless" as anonymous referencing practices can sometimes do (Duncan, 2017). I did not find one single solution to this issue but chose to use a different naming practice to be appropriate to the context of each participant group. For the school students and the women who received the DfG Kits, I used the name of the boarding home or community of which they were part. I had planned to use participant-chosen pseudonyms, but these girls and women seemed to be uncomfortable thinking of another name. For the women who sew the DfG Kits, I used initials because each of them had Burmese-style multi-word names and again, did not want to use pseudonyms. Partners staff were referred to by role apart from the DfG Programme Coordinator (pseudonym: Sarah), Assistant Coordinator and my main translator (pseudonym: Htoo Htoo) and the 'Care' Coordinator (pseudonym: Eh Moo), as they had key roles in the programme and were mentioned several times.

\subsection{Conclusion}

This chapter has discussed my epistemological and methodological influences as well as the research route I took. My research was influenced by a feminist epistemology which values women's experiences, voices and contributions and encouraged me to address values of power and knowledge creation, which I speak to in Chapter 8. I chose to adopt principles of Appreciative Inquiry as a way to honour the strengths of the programme, the women involved and my relationship with the staff. Additionally, I was influenced by the 'Follow the Thing' approach, which directed the way I have chosen to describe the programme in Chapter 5 - focusing on process and connection. Despite the challenges of working in a complex field environment, the use of a mixed methods methodology including mapping helped me to generate reliable information and a wide range of ways to represent participants' voices, which will be explored in the following chapters.

In this chapter I have also dealt with practical and theoretical issues of analysis, ethics, power, accessibility and translation. The complexities of working cross-culturally and in an unknown context have necessitated that I work as collaboratively as possible, stay reflexive and responsive, and acknowledge that the research is limited and partial. 


\section{Chapter 5 - The MHM Programme}

\subsection{Introduction}

This chapter follows the 'journey' of the menstrual hygiene programme and the Days for Girls Kit (Cook \& Tolia-Kelly, 2010). It is intended to be an appreciative summary of the processes involved in production and distribution with a people and systems-based focus. The structure is inspired by 'Follow the Thing' stories, which track "multiple sites of observation and participation that cross-cut dichotomies such as the "local" and the "global," the "lifeworld" and the "system."' (Marcus, 1995, 95). The first section describes the foundations of Partners' MHM programme as well as the systems it works within. The second section describes the chain of production of a Days for Girls Kit, from materials to construction to the places of distribution. In light of this process, the third section attempts to draw connections between the women who are located at each site, and recounts how women see themselves as connected through their contributions to positive MHM outcomes for women and girls.

\subsection{Structural location}

\subsubsection{Partners Relief \& Development}

The programme's coordinator, Sarah, has been working with Partners Relief \& Development from its early years. Friends with the organisation's co-founders, and having spent parts of her childhood in Thailand and Southeast-Asia, Sarah moved to Partners 15 years ago and began working on a sewing training project in Thailand. When the DfG sewing project was introduced to the team, they envisioned what the kits might look like in a Thai/Burma context and how they could help employ women who needed jobs to make them. While Sarah had a limited sewing background, she enlisted the help of two women employed in the sewing project, and they "worked it out together".

During the course of my fieldwork in Thailand, the Partners MHM Programme moved departments within the organisation. It had originally been under 'Education', reflecting its focus on training women to sew and its MHM education aspect. But under a newly formed department, 'Livelihoods', programme directors believed its focus on women's employment would come to the fore, while still encompassing the health, wellbeing and education aspects of the programme. Within the Livelihoods department, the DfG programme would now sits beside and connected with Partners' sewing and weaving programmes that train women and sell products as a means for fundraising.

I was fortunate to be a part of the planning and strategy sessions for this new department, and wrote down some of its working statements: 
Vision: Empower and develop communities through sustainable livelihood training and support

Mission: Demonstrate God's love through livelihood training to increase food stability, health and income generation

The strategy included goals to "train women from the migrant community to sew DfG Kits" and to "produce 2000 DfG Kits by 2020". There was also a vision to "extend sewing training into three areas to enable skill and income generation for women." Despite funding restraints, there was a general feeling that staff wanted to consistently produce DfG Kits to help women and girls during menstruation and to support as many women as possible into purposeful employment.

\subsubsection{Days for Girls International}

The programme is situated in a 'world system' (Marcus, 1995) of 'Menstrual Liberation' where popular interest in menstruation has been increasing (Bobel, 2019; Rador, 2017). Within this movement, many NGOs who have been promoting 'menstrual activism' have received wide support from donors (Bobel, 2019; Days for Girls International, 2019b). The Southeast Asia Programmes Coordinator at Partners confirmed the current positive attention for menstrual health initiatives, "It's one of the projects that we get the most comments on. Women [donors] can relate to it" (D. Gibbons, personal communication, 2019). Days for Girls International is one of the largest MHM organisations and mobilises programmes across over 140 countries (Days for Girls International, 2019b). Partners' decision to connect in with the organisation would in theory give them access to the forefront of MHM kit design, MHM research, and a global network of MHM practitioners.

At the start of Partners' MHM programme, Sarah said she found contact with Days for Girls difficult. Being the only team located in Thailand at the time, there were few existing networks close by and Sarah struggled to gain constant contact from Days for Girls International staff members, "so we just did our own thing". They used the resources available online, sourced materials where they could and started to produce Days for Girls Kits. After finding a Days for Girls International contact in Southeast Asia, the Partners programme was able to properly connect in with the international system. At the time of research, Partners was sending reports to the organisation and conferring with other teams that have emerged around the region.

But Partners' programme is unique within Days for Girls International (Figure 5.1). The international NGO has two overall structures which produce DfG pads: Teams and Enterprises. Teams are groups (often from higher-income countries) of volunteer menstrual health activists who sew kits to donate to Days for Girls International. Enterprises are part of the 'Days for Girls Social Enterprise Program' they are locally-led and employed and sell their kits to generate income (Days for Girls International, 2019a). The Partners programme is officially a 'Team', but they don't run like the volunteer groups. 
They employ local women to give them skills and an income, but they do not sell the DfG Kits. Their costs are funded by Partners and programme-specific donations, and they give the kits to the communities where Partners works. As Sarah put it: "We are unique, because we hire women... And we give to those who can't afford to buy them... As far as I know we're the first one to ever do it the way we're doing it."

\begin{tabular}{|l|l|l|}
\hline Teams & $\begin{array}{l}\text { Partners Days for Girls } \\
\text { Programme: }\end{array}$ & Enterprises: \\
\hline Volunteers sewing kits & $\begin{array}{l}\text { Trains and employs local } \\
\text { women in low and middle- } \\
\text { income countries }\end{array}$ & $\begin{array}{l}\text { Trains and employs local } \\
\text { women in low and middle- } \\
\text { income countries }\end{array}$ \\
\hline Kits are donated & Kits are donated & Kits are sold \\
\hline $\begin{array}{l}\text { Led by women in mostly high- } \\
\text { income countries }\end{array}$ & $\begin{array}{l}\text { Led by local and foreign } \\
\text { women in Thailand }\end{array}$ & Led by local staff \\
\hline Self-funded & $\begin{array}{l}\text { Self-funded (through } \\
\text { Partners) }\end{array}$ & $\begin{array}{l}\text { Financial support from Days or } \\
\text { Girls International }\end{array}$ \\
\hline
\end{tabular}

Figure 5.1: Aspects of MHM programmes - comparing Teams, Enterprises and the Partners DfG Programme

While the Partners MHM programme doesn't fit seamlessly into Days for Girls International, Sarah was proud of the way her team could adapt to the specific needs of Mae Sot's location: employment for women and menstrual health relief and education.

Despite practical challenges to this connection, I did notice in my interviews that participants used similar discourse about MHM and its effects as seen in Days for Girls. Prahnee, the Communities Coordinator in Mae Sot who has been part of DfG distributions to migrant women, described what she saw as the reasons behind Partners' adoption of DfG:

Before, Sarah, has a friend... And she had been working with the DfG programme in Africa - because in Africa, there are a lot of women who will not go to work or school... and they think it is good to run the programme in Thailand too. This is when they start to run the programme. And if we think about it, Burma is the same as Africa. Many people from Burma don't have things to cover when they have their period. It is very useful for the people in Burma.

\subsection{Following the DfG Kit}

The connecting factor between each part of the DfG programme process was the DfG Kit itself. During its production and distribution, the kit and its pieces passed through many pairs of hands, each with stories that marked and were marked by the DfG programme. The process locations below are marked on Figures $5.2 \& 5.3$. 
$\downarrow \quad$ The US: Flannel and waterproof PUL lining are sourced from various locations in the US. Much of the programme's funding also comes from donations from the US (as well as some from NZ, Australia and Europe).

$\downarrow$ Chiang Mai: Underwear and some cotton is bought at shops in Chiang Mai. Materials and some completed bags pass through the Chiang Mai office.

$\downarrow \quad$ Mae Sot: Cotton is sourced from shops in Mae Sot.

$\downarrow$ Mae Sot workshop: Women work to sew DfG pads in the Mae Sot workshop.

$\downarrow$ Distributions: DfG pads are distributed to Mae La refugee camp, Migrant communities in Mae Sot, and other locations outside of Thailand (not included in this research project).

This section maps the chain of production of the DfG Kit within the Partners DfG programme, and how in each step, women contribute to meaningful MHM outcomes for women and girls in Thailand.

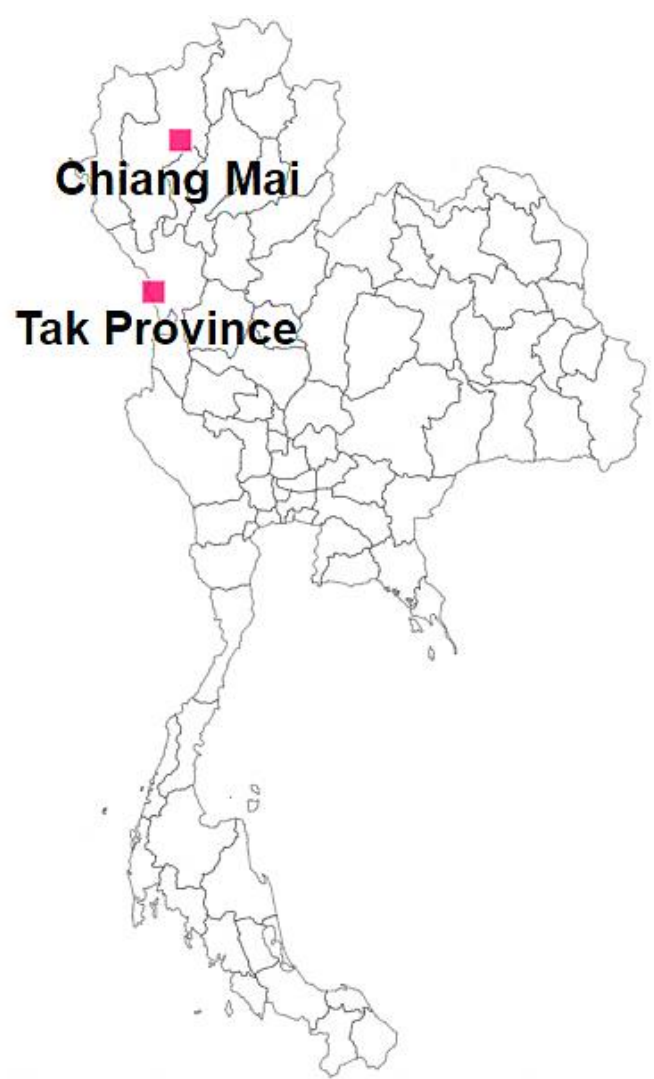

Figure 5.2: Map of Thailand: Locations of the DfG Programme

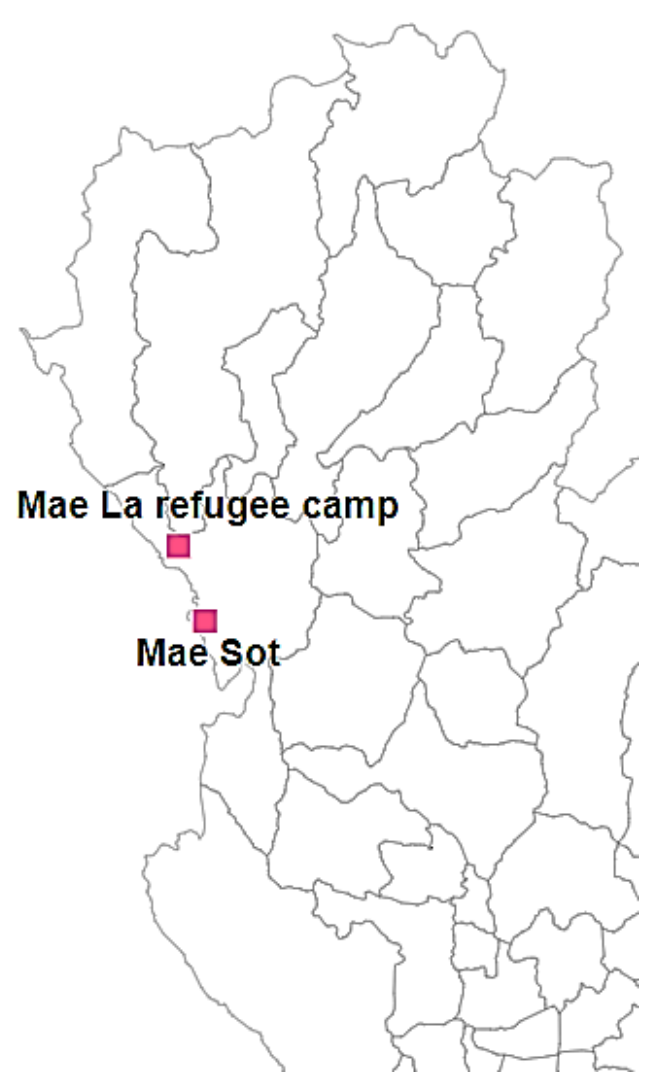

Figure 5.3: Tak Province: Locations of the DfG Programme 


\subsubsection{Sourcing materials}

Partners sourced materials from a variety of places for the DfG programme. The small 'Days for Girls' patches were supplied directly by Days for Girls International and the snap closures for the 'shields' came from the United States.

Some materials could be obtained in Thailand - Sarah and Migrant Communities Coordinator, Prahnee had relationships with local shops and suppliers where they could buy cotton, some flannel and underwear to make the kits (as seen in Figure 5.4). There were challenges to obtaining these products. For example, the programme could not predict exactly who will be receiving the Days for Girls Kits and so the size of the underwear in the packs would not always fit the woman or girl receiving it. Illfitting clothing from humanitarian donations is a common problem, and it is recommended that practitioners have as best communication possible with recipients as well as offering them plenty of choice (Gaugele, 2020). Sarah bought a range of sizes for the kits. She said, "I just hope they can swap them around between each other when they get them".

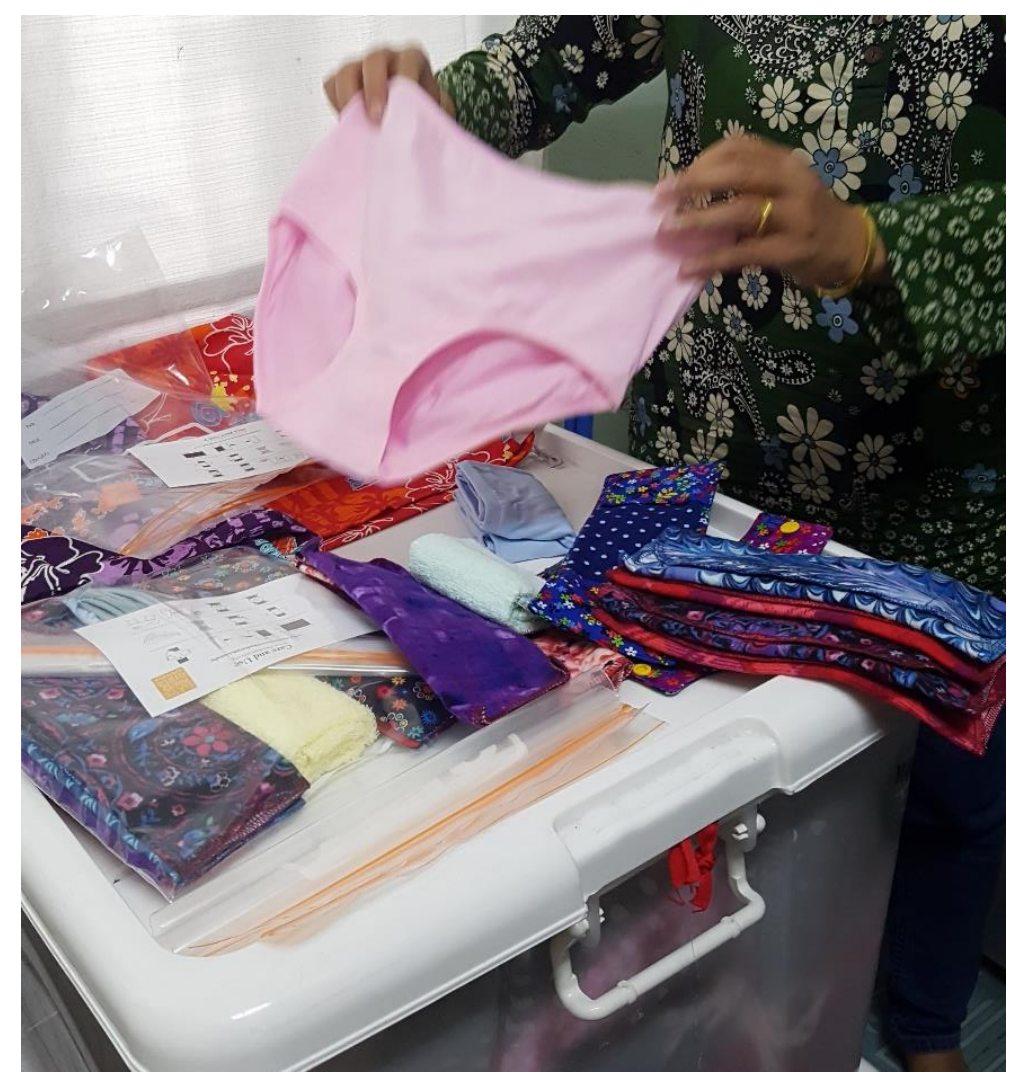

Figure 5.4: The materials that make up the DfG Kit including underwear, cotton and flannel.

For the liner that is folded into the menstrual shield to absorb blood, the team looked to two places. For the back piece, flannel could be sourced from various locations in Thailand. This material was not as soft as the front and "pills easily" according to one of the women who sew them. So, for the front of the liner, Sarah asked for good-quality flannel to be brought over from the US. While she would do this 
herself when she made trips to visit family and friends, a large portion of this flannel was bought over by Partners 'Teams'. These were groups of volunteers or visitors to Partners Thailand who would often come from the US. If they agreed to bring flannel over with them, Sarah would send them specifications for the purchase and information about the Days for Girls programme. While Partners offered to pay the teams back for the fabric, they often decided to donate it instead. Sarah explained of these teams that "they say they have prayed about it and that they want to".

The PUL lining, which goes inside the 'shield' as a leak-proof barrier, was also sourced from the US. Sarah said this is the only place she could find the right fabric that was both breathable and waterproof. She said that she would like to know where other teams within Days for Girls International source this lining.

In this programme, sourcing material was a collaborative process involving people from a range of locations. The involvement of people as far away as the US in the material sourcing could be a way for donors to feel more connected to the project in a way that goes beyond giving money although the programme hoped to source materials more locally if they could find appropriate options.

\subsubsection{The sewing workshop}

There were five women employed by Partners to sew the DfG Kits in the sewing workshop (workshop pictured in Figures 5.5 \& 5.6) Their work ranged from a few days a week to full-time and each had a role in making the kits. The roles varied in difficulty and were usually allocated depending on how long a woman had been at the workshop and whether she had previous sewing experience. While the drawstring bags were beginner-level, the shields required the use of a die cutting machine and were sewn by the more advanced staff. Some women also became 'trainers', teaching other staff and women from outside of Partners.

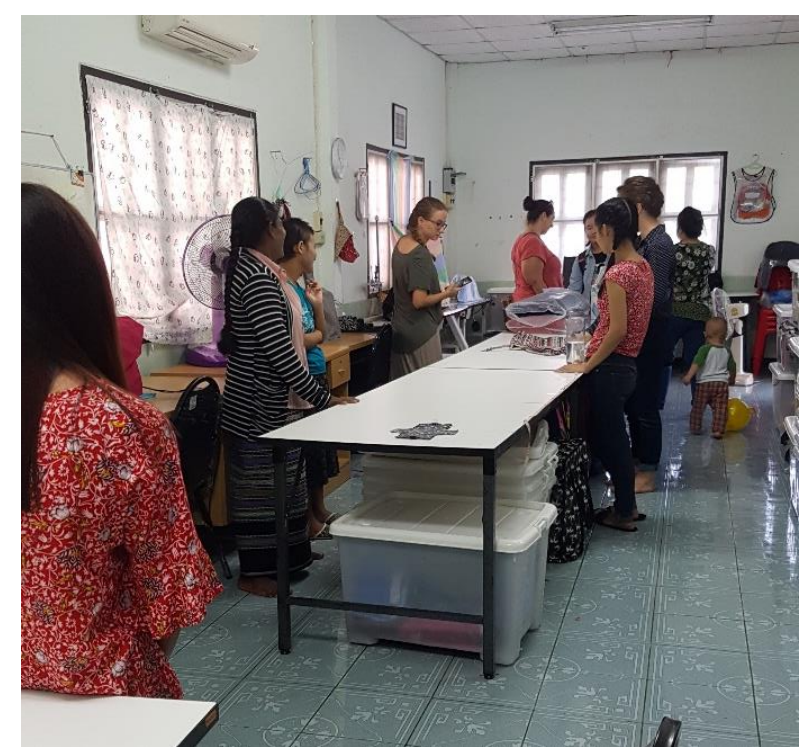

Figure 5.5: The sewing workshop

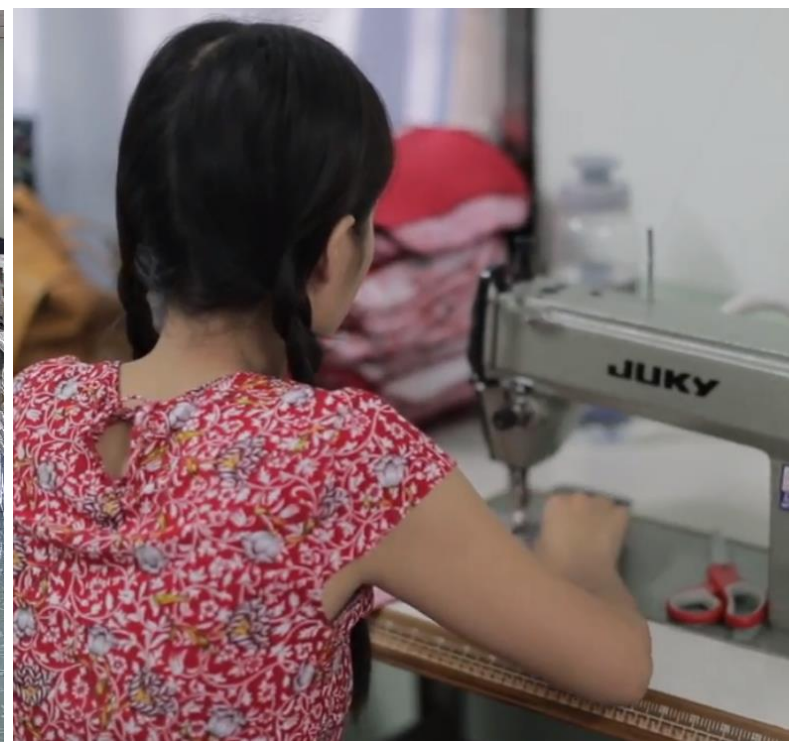

Figure 5.6: DfG Kit sewist (Source: Partners Relief \& Development) 
NKP, from Kaw Po Ple village in Karen State, explained her journey from training to advanced sewing to me:

TB: How long have you worked in this programme?

NKP: For Partners, for two years, and before that I did training... At first, I just learnt to sew dresses and bags and pants and skirts.

TB: And now you make the shields?

NKP: (Affirms) I cut the plastic, and put two or three layers together.

As NKP described, each of the women learnt to sew items of clothing other than the DfG Kits. They valued the ability to sew helpful items for themselves and their families, and some brought these skills to trainings held with women in their own villages in Burma. Others said they would love the opportunity to do the same.

\subsubsection{Distributions to Mae Sot}

Distributions were carried out by various Partners staff, sometimes alongside foreign teams. Those leading the sessions had to have completed the Days for Girls Ambassador of Women's Health online training programme. This is course would qualify distributors to teach the MHM and women's health education with a flip-chart alongside distributions of DfG Kits.

In the Mae Sot migrant community, distributions had been carried out in schools and in a local church (Figure 5.7). The Migrant Communities Coordinator worked to assess needs in the community and where and for whom the DfG programme would be most useful:

I take care of the communities. And at the same time, I also work for the sewing with Pipi. I will prepare and arrange training and I find jobs for anyone who wants to work here... And I manage the orders - how many they need for different places. 


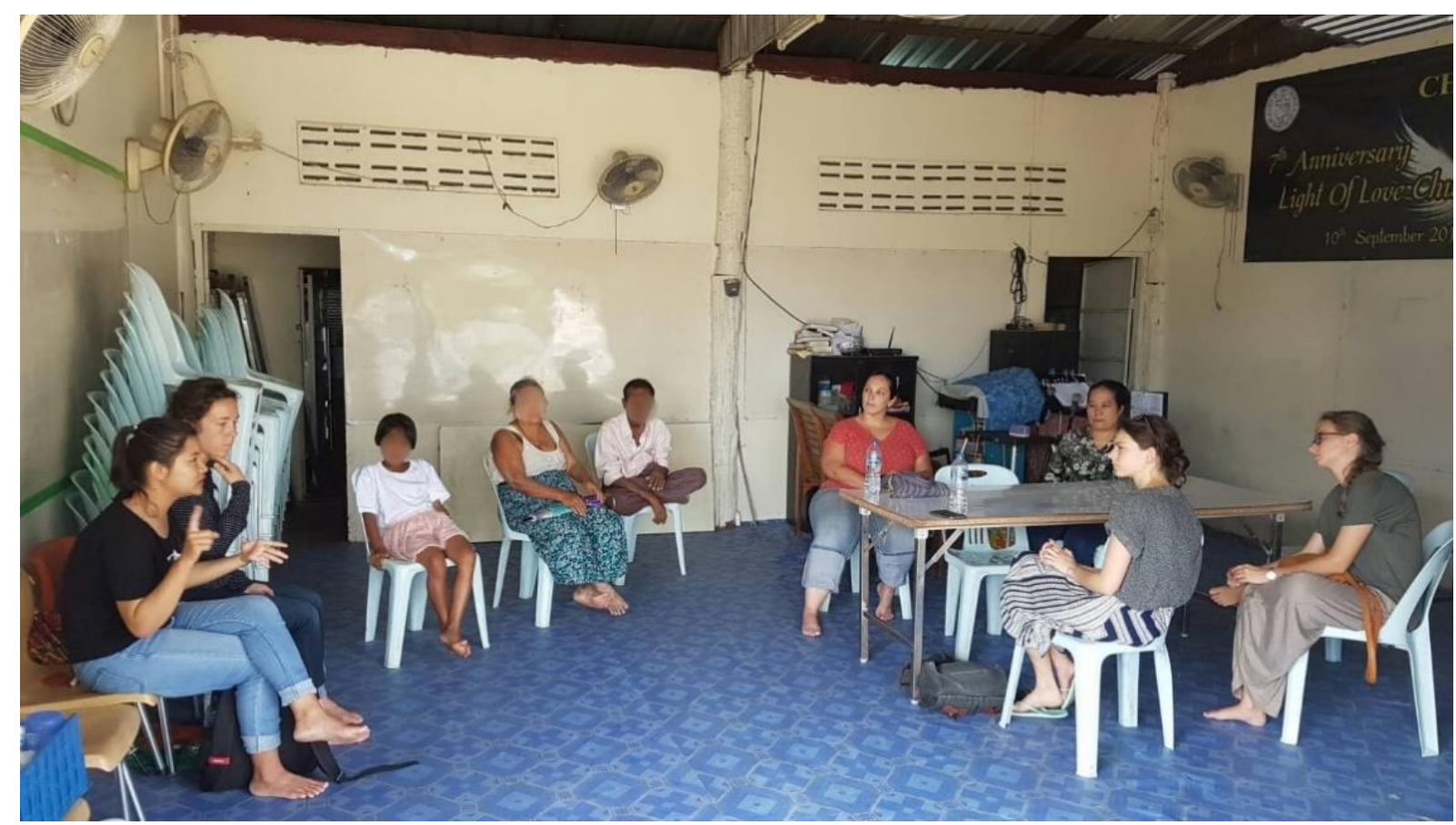

Figure 5.7: Partners staff, church members, visitors and myself (second right) at Light of Love Church, DfG distribution site

A woman I interviewed from the migrant community described her experience of the distribution in her local church:

MCW: The team [...] came and explained - give the training, and hand out kits. They just explained like how to put the kit onto the underwear, you know, how to clean it and how to, like, what we need to do the process.

\section{TB: How did you feel when you first got the DfG Kit?}

MCW: It's very useful and very thankful because, like, after she got DfG Kit she doesn't need to buy pad from the store anymore."

It seemed that the MHM programme was integrated well within not only Partners but the communities they had built and supported.

\subsubsection{Distributions to Mae La}

Inside Mae La refugee camp, Partners supports four boarding homes: International Rescue Committee (IRC), Mission, Htee Moo Traw and Ko Thu Lu (Figure 5.8) . Most students at these homes have family living inside Burma or at other refugee camps and live in the homes full time. In these spaces, students are looked after by caregivers and take part in chores and cooking to keep the homes running. Partners began DfG distributions in Mae La after a member of staff in one of the boarding homes asked for this kind of support. 


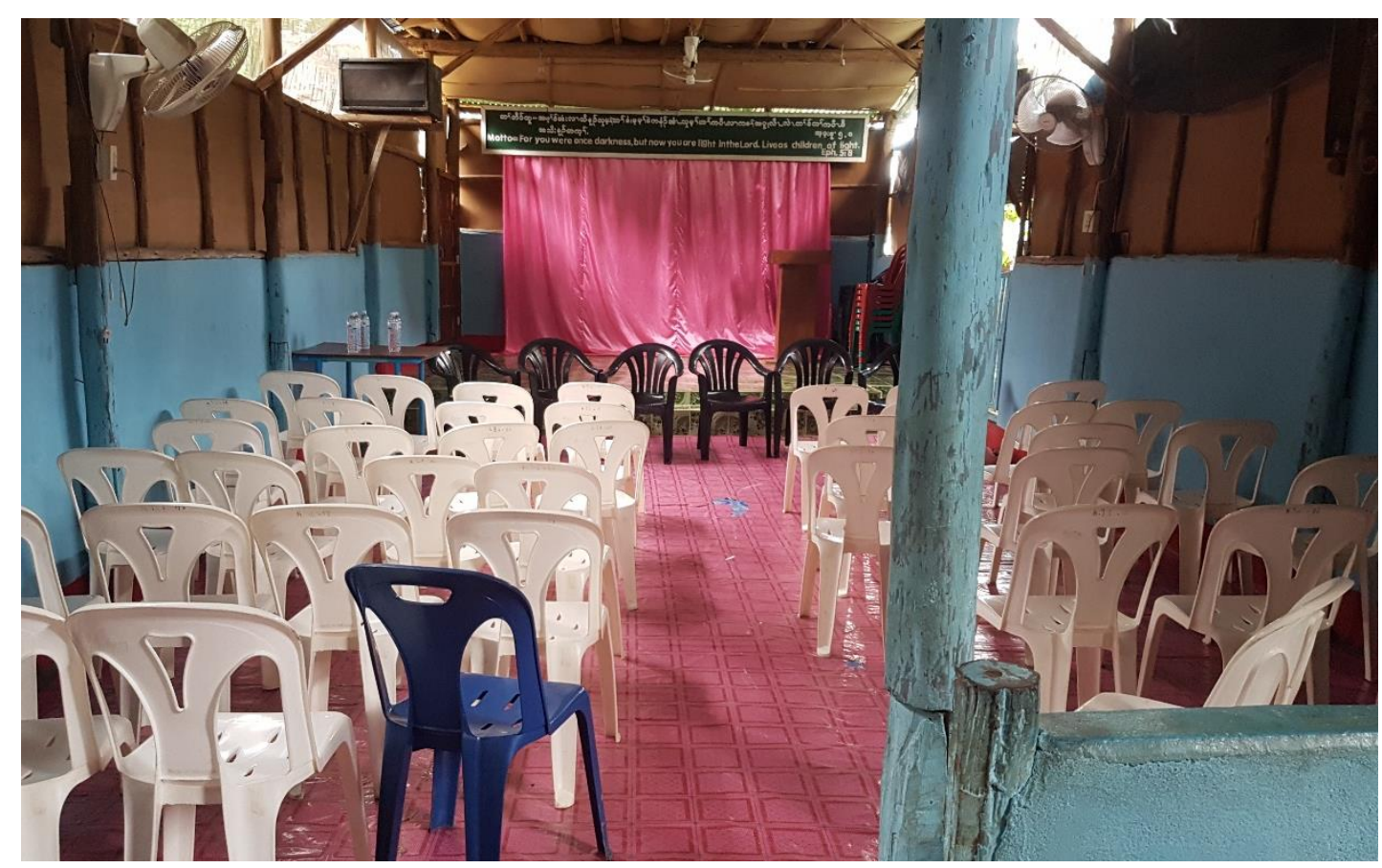

Figure 5.8: Ko Thu Lu boarding home hall, where Partners distributed DfG Kits

Partners staff carried out distributions and the DfG training in classrooms or school halls in each boarding home with the female teenage students. In my interviews, I asked students to describe the time when they received the DfG Kits. They recounted the experience as being informative and helpful to their knowledge of the DfG Kit: "Spring Song and Eh Moo came to show us how to use them, how to wash them, how to dry them" (Htee Moo Traw student). As in this quote, the students referred to the names of staff far more often than 'Partners Relief \& Development' or 'Partners'. This personal connection will be explored later in the chapter.

\subsubsection{Where/how do women and girls use the DfG Kits?}

For most of the women and students I talked to, the DfG Kits fitted well into the rhythms of their daily lives. During menstruation, the migrant women I interviewed said they wore the kit to their jobs at the sweater factory (Figure 5.9) and the students said they wore the kits to school. Students also said they wore the kits for other activities - playing football, going to church or to the shops and visiting friends. While some took a while to get used to the kit after the distribution, they said over time they became comfortable:

When we first saw the kits, they looked strange for us how to use them. Later on, we were fine and knew how to use them well. For the first time for using it, it is not comfortable and it is like something between our thighs is big or stuck. When we use it many times, we are used to it and feel comfortable. (Ko Thu Lu student) 


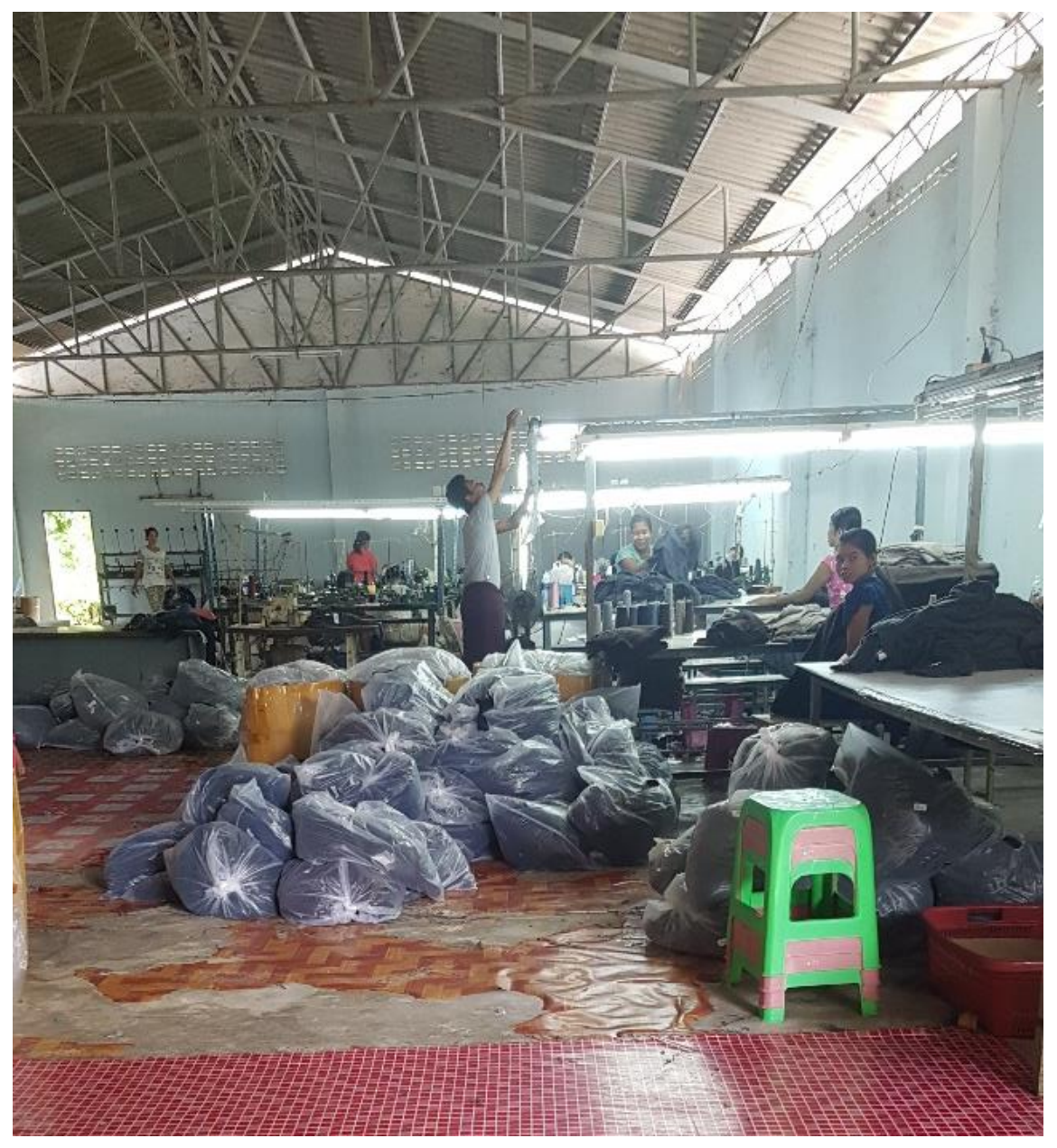

Figure 5.9: Factory in Mae Sot where migrant women make sweaters

The participants would wash and dry the kits in their homes and boarding homes (Figure 5.10). They had established routines that fitted in with their spaces: "We wash and dry them in the dormitory. There is enough space to hang them. In the rainy season, we dry them outside first. If the water is too much, we dry them underneath the house" (Htee Moo Traw student). 


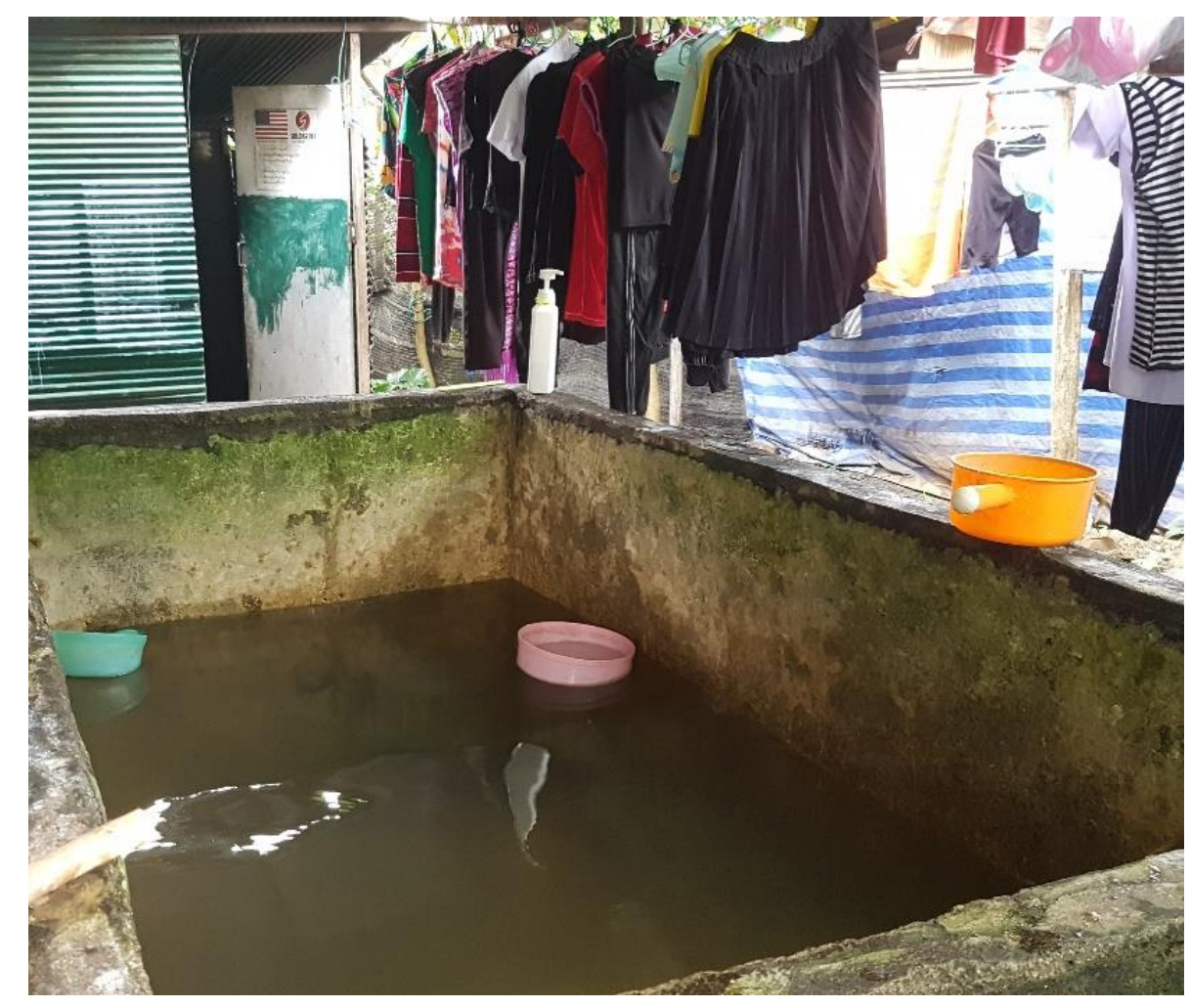

Figure 5.10: Washing and drying space in Ko Thu Lu boarding home

The DfG Kits, in their 'destination' with the user, had become integrated aspects in the menstrual lives of the students at Partners' supported boarding homes and for the migrant community women who use them.

\subsection{Connections}

Partners Relief \& Development sees the DfG process as intimately connecting women, girls and those invested in the programme. In their 2019 "The Summer of Beautiful” Journal, Partners board member Jen Tendero wrote a very 'Follow the Thing' -styled article - connecting the donors to the employees to the recipients of the DfG Kits. She introduced the production chain as follows:

This is love: a motley group of people - most of whom will never meet each other - linking arms to form a supply chain that reaches into wallets, runs into fabric stores, flies over an ocean, feeds through a sewing machine and motors over mountains and through the gates of a refugee camp straight into Naw Khin Mar Cho's [a participant in their story] tiny bamboo hut where her husband cradles their baby while her mama walks to the market because now she can. (Tendero, 2019, p. 35).

While emotive to capture donors' interest, these words are descriptive of what Cook \& Harrison (2007) call "an amazing array of complex connectivities and mobilities" that can be found in a single production chain (p. 58). Although the participants along the journey of the DfG Kit may not know much about each other, they are part of "a social system" that sees "commodities as ways of connecting together the 
lives of people who don't know anything about each other, but whose lives are intimately connected on a day-by-day basis." (Cook, 2016, 1:50).

This section will discuss a few of the ways that staff, sewists and recipients of the DfG Kits connected (or didn't) and how they saw these connections themselves.

\subsubsection{Partners Staff}

Within Partners, there were several members of staff who were connected through the Days for Girls programme. Those that worked with the sewing women were in communication with those that managed distributions and most issues through the production line seemed to travel to the appropriate places. Staff told of their flexibility and willingness to help out in the areas of the DfG programme even if their work did not directly relate. Eh Moo, from the 'Care' team who coordinated with the boarding homes, said, "We don't have exact roles... we help with each other if we need. And if they ask us to give training, we just go and give training."

Some Partners staff also had an important personal connection with the participants in the programme - they used the Days for Girls Kits themselves. The Migrant Community Coordinator said during our interview, "I use DfG and the DfG Kit. I just feel that when I use the DfG Kit, it's very nice. Before that, when I used the pads, I would feel a lot of spots come out (leaking)." She talked about the kits positively and with similar words to the other users I interviewed. It seemed that she was confident of the difference a DfG Kit could make for the communities where she worked, because she had seen that difference in her own life.

\subsubsection{The women who sew the DfG Kits}

In my interviews with the women who work in the DfG workshop, I asked participants of their knowledge of DfG production and consumption. I wanted to know how they saw their role in the bigger chain of the programme.

Overall, the women who sewed the DfG Kits seemed to know a fair amount about the places the kits go to. While one sewist (MM) at first said she didn't know a lot about recipients, ("I just know they go to Partners and they pass them on to the women"), she later talked about the women in the migrant community who received the kits, telling me that they used to use disposable 'Kotex' brand pads but it is much better now they use the DfG Kits. NKP had a clear idea of where the kits went after her team produced them: "Partners comes and takes the materials that they need and sends to inside Burma or along the border, to those who really need it. For girls who cannot afford expensive things."

The women spoke positively of the MHM kits and their effects on their community. MKO said, "I heard from other people that they think DfG Kit is very nice. "It's big, safe, will hold your blood" - Very good feedback." MM also praised the kits: 
MM: If we use this one, it will be not hot - it is cool. It is also comfortable to use. They can cover all your blood. But not for Kotex. If you use them, it is hot and it becomes big on your hips.

TB: Do you use the DfG Kit to manage your menstruation as well?

MM: Yes, I use this one. It's a good kit.

Two of the women told me of a special connection they had within the programme: they had been able to distribute kits and provide sewing training themselves to women in the villages they came from in Karen State. MM shared this experience with me and how she hoped it would make a difference for women and girls in her village:

When I brought back the kits to my village, I taught the people how to use them, and for those who share these things to the school, like their teachers, maybe they can train them...

I gave them to the women in my village. Like young ladies and students.

Through talking to the women about these events, it became clear that the opportunity to distribute kits and train women in their home towns gave them a sense of purpose in their job sewing the pads. NSL felt this, especially of the sewing training:

It's really important to tell the women from my village because they need to... they are not really rich... some women are poor, so they cannot afford... and those from the shop, it's not so good for their protection. And if they can sew by themselves, they can save their money for their families.

This sense of purpose came across as one of the most important aspects of the sewing work for the women I interviewed. I will re-visit this idea and its implications when I explore the effects of the work on the sewing women in the next chapter.

\subsubsection{Students}

The students I interviewed from the boarding homes seemed to connect their experience of the distribution and training to the staff or volunteers from Partners that were present.

While students had a harder time remembering the non-Karen volunteers ("A foreigner came, we cannot remember"), many could tell me the Karen staff who came by name. The Ko Thu Lu and IRC students I spoke to seemed to particularly connect their experience to the 'Care' coordinator, mentioning her name several times as they told of the distribution and the MHM education. They said she was very helpful in answering their questions. 
I was particularly interested in this connection because Eh Moo had been a student at IRC boarding home in Mae La as a teenager. In her story, she told me of the close connection she had to the school and to Mae La:

I came when I was 13, after my Dad passed away, I came to the camp. My Mum passed away first, when I was 1 and $1 / 2$ year old. My uncle told me about her. From my dad, it's almost just 12 years, and then when I was 13 and I moved to Mae La Camp, and stayed in the dormitory with Partners support.

Eh Moo spoke fondly of the caregivers and said that in Mae La "I feel like at home". She told me that she believed her connection to the students in this way helped them to open up about MHM matters during the training:

\section{TB: Do you think that the DfG training makes them feel more confident?}

Eh Moo: Yeah, yeah yeah. They speak out. For me, I used to stay at the dormitory - they feel like I'm friends with them, so they are no more [shy], they just feel free to ask and to talk to me.

Interestingly, the students who spoke of the DfG programme staff by name in our interview were also able to recount to me more of the training they had retained. This may have been due to how long ago they had had the training, but it may have been to do with their connection with Eh Moo and the Partners staff.

There is strong support for employing local staff as educators and leaders of education in 'developing countries' (Brown, 2015). As well as having a "situated knowledge" (Haraway, 1991), local staff are in the best position to be building meaningful relationships as they have fewer communication issues, cultural boundaries and differences of experience to cross.

\subsection{Conclusion}

This chapter has demonstrated how participants contribute to the processes involved in the Partners DfG programme to promote positive MHM outcomes for women and girls. The process includes and connects women from different nationalities, ethnicities, ages and physical locations. It is embedded within Partners' structures, connected to the MHM 'world system' through Days for Girls International, and therefore influenced by 'menstrual activism' discourse. The process involved is collaborative and allows for knowledge-sharing between women. It involves wider communities and staff have ongoing connections to participants in the programme. My research shows that the connections helped the women who sewed DfG Kits to feel more purpose in their work, and those who received the kits to make the most of the programme. 
This chapter has been an attempt to set up a "fuller story" (Cook, 2004) of the DfG programme. The evidence, analysis and reflections that follow can only be accurately understood in context. The 'effects' I will discuss are not from the DfG Kit alone, or an unhuman 'programme', but by a chain of women working hard and collaborating for menstrual freedom and employment that empowers. 


\section{Chapter 6- Kit uptake and effects}

\subsection{Introduction}

As mentioned in Chapter 1, this study endeavours to understand the ways Partners' Menstrual Health Management (MHM) relief affects confidence and agency for refugee women on the Thai-Burma border. Drawing on surveys and interviews carried out with Mae La students as well as interviews of some migrant women, this chapter explores the impact of the DfG Kit: which participants use it, why they use it and how the use of the kit affects their sense of confidence and agency. It also explores how the work making the DfG Kit affects the lives of the women who sew it.

I start with a quantitative look at 'kit uptake' and a brief review of what students in Mae La used before they received their DfG Kit. I then examine the possible reasons behind a high uptake using students' descriptions of the kits' positive effects. The chapter ends with a similar discussion from the view of migrant women in Mae Sot who also received DfG Kits, and with the experiences of the migrant women who sew the kits. For both the students and the migrant women, effects are categorised into two overall concepts: confidence and agency.

This chapter integrates some reflections and connections to MHM literature but a more in-depth analysis and critique takes place in Chapters 7 and 8.

\subsection{Mae La Students}

In Mae La refugee camp, I surveyed 68 students who had received DfG Kits in the four boarding homes Partners supports. The boarding home staff had organised for the students who had been part of the distributions to be present, so I estimate that I surveyed between $90 \%$ and $100 \%$ of those who had received DfG Kits and were still in school. This high sample size is helpful because it can more accurately detect trends for the total population (Murnane \& Willett, 2011).. Respondents were aged between 13 and 22 and most had received a Days for Girls Kit in the last 18 months.

The questions asked (Figure 6.1) were based on the survey from Days for Girls International that tries to understand the effects of and feelings towards the DfG Kits of students worldwide (Days for Girls International, 2019c). They aim to understand aspects of confidence, comfort, ease of use and mobility. 


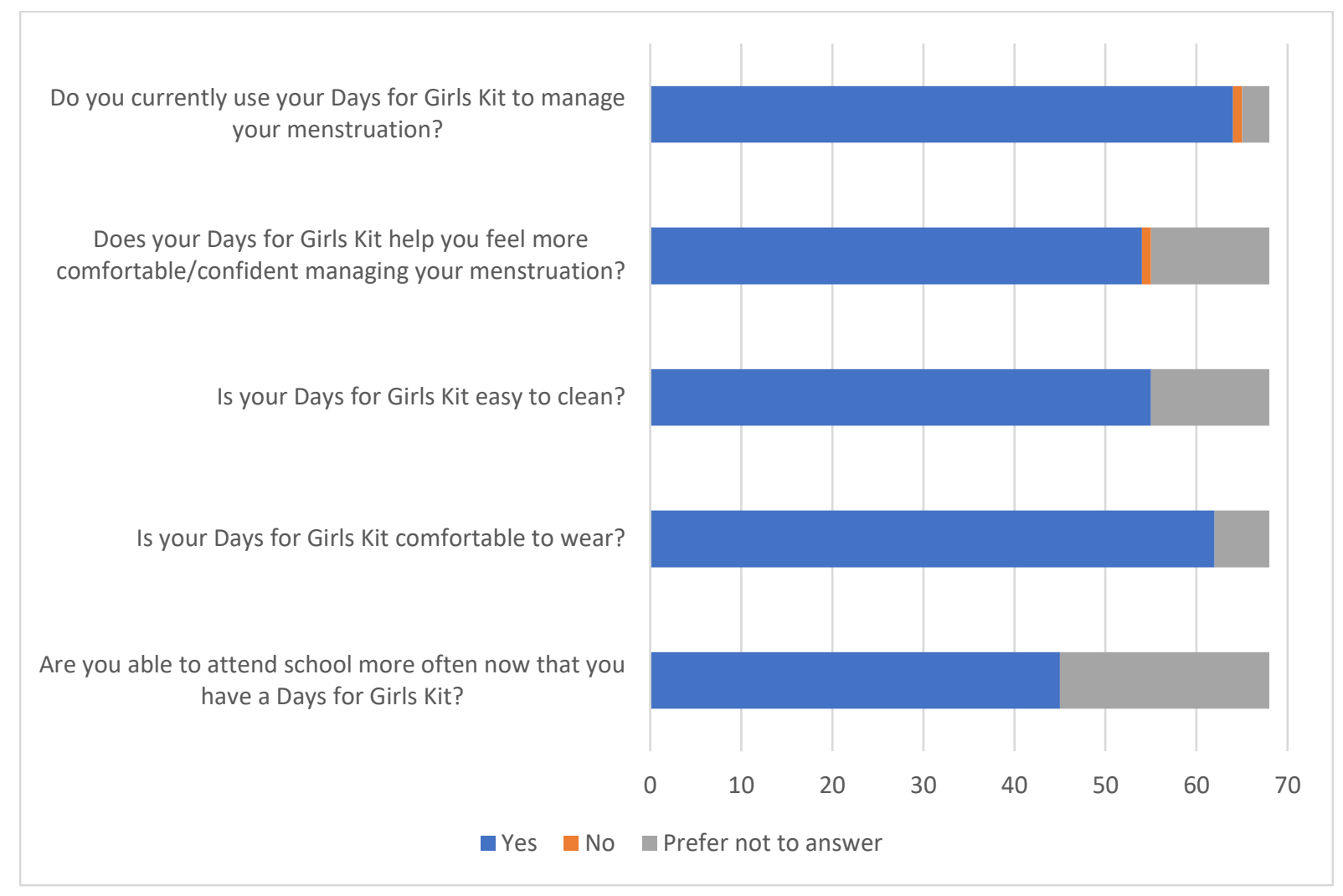

Figure 6.1: Mae La Students Survey Results

Out of the 68 participants surveyed who had received DfG Kits, 64 said that they use the kit to manage menstruation some or all of the time (Figure 6.1). One student said that they use only alternative measures and three preferred not to answer. This is a very high self-reported uptake for the Partners DfG programme - 94.1\% - and suggests that the kit was well received in this community. Some reasons that students use the DfG Kit will be discussed in further sections of this chapter, using interview data and other statistics from Figure 1.

\subsubsection{A note on these findings}

As evident in Figure 1, there are far more students who responded to questions with 'I prefer not to answer' than 'no'. This result could have been influenced by several factors. Firstly, students who didn't understand the question may have preferred to abstain from answering. Secondly, there was a 'yes/no' binary of answers offered, so if students had a neutral response they also may have preferred not to answer. Lastly, the concept of 'grangai' (Thai) or 'saving face' could have affected answers - the idea within several Southeast Asian cultures of not wanting to say something that will displease or reflect negatively on another (D. Kahn, participant interview, August 23 2019; Merkin, 2018). It is possible that those who would have answered 'no' chose to abstain from answering instead as they didn't want to reflect badly on Partners, the DfG programme or the people who gave them the kits. 
Taking into account this effect, it is possible that the data set skews positive. There may have been more students answering 'yes' than would reflect reality and participants may have been motivated to answer more positively than they felt in interviews. But in keeping with feminist epistemology, where participants and women's voices are expert (Reinharz, 1992), this study will be largely based on the self-reported results. I took steps to mitigate the effects of this potential bias by working with Htoo Htoo to make questions clear, emphasising anonymity and encouraging students that all answers, positive or negative, were helpful to Partners Relief \& Development.

\subsubsection{What did students use before they received the DfG Kit?}

In interviews, some of the participating students were asked about the previous measures they used to manage their menstruation. Some students spoke of what they did before coming to Mae La:

Before we came here we used sarongs only, we would stay home and not go to school if we had a heavy period. No pads, we wear many pants, we would not go to church because they are worried that people are going to see. (IRC student)

Eh Moo recalled what students at Mae La did before Partners DfG programme began in the camp. She said that they were provided with underwear and fabric through the Karen Women's Organisation:

One of the organisations called KWO (Karen Women's Organisation), they give us like... fabric, and it's a little bit thin, and they give us 2 or 3 underwear. And (she laughed) at that time the underwear is so big! [...] Most of the foreigners, their sizes are bigger than the Asian people. And then they give us fabric - 10 or 15 inches, and then we have to cut it with the scissors into square shape. And if our period is coming, we fold 3 or 4 pieces... and put it in the underwear.

But when current students came to Mae La, they said that they had access to disposable pads. The most popular brand mentioned by participants was 'Kotex': "Before, we used Kotex, everyone else used to use them too. Partners donated packs. There was enough disposable pads for everyone" (IRC student). Unlike in some representations of MHM programme-less girls in low-income settings (Goonj, 2018; Moyo, 2018), these students were not relying on scrap-cloth or leaves to catch their menstrual blood. This solution may not have been ideal, but their period management was similar to that of menstruators in most other countries. For most students surveyed, their transition had been from disposable pads to the DfG Kit. Therefore, the high uptake does not necessarily indicate that students now have access to menstrual care where there was none, but rather that most students have found a preferred way to deal with their period. 


\subsubsection{Comparison with Shan students}

A similar, separate study was conducted in a different location on the Thailand/Burma border with high school students from Shan State. The results of this survey were considerably different. Out of the 19 Shan students surveyed, only $42 \%$ said that they used the DfG Kit (Figure 15). The other 58\% said they preferred to use alternative ways of managing their period. This is compared to a $94.1 \%$ of reported usership in Mae La (Figure 6.2 \& 6.3).

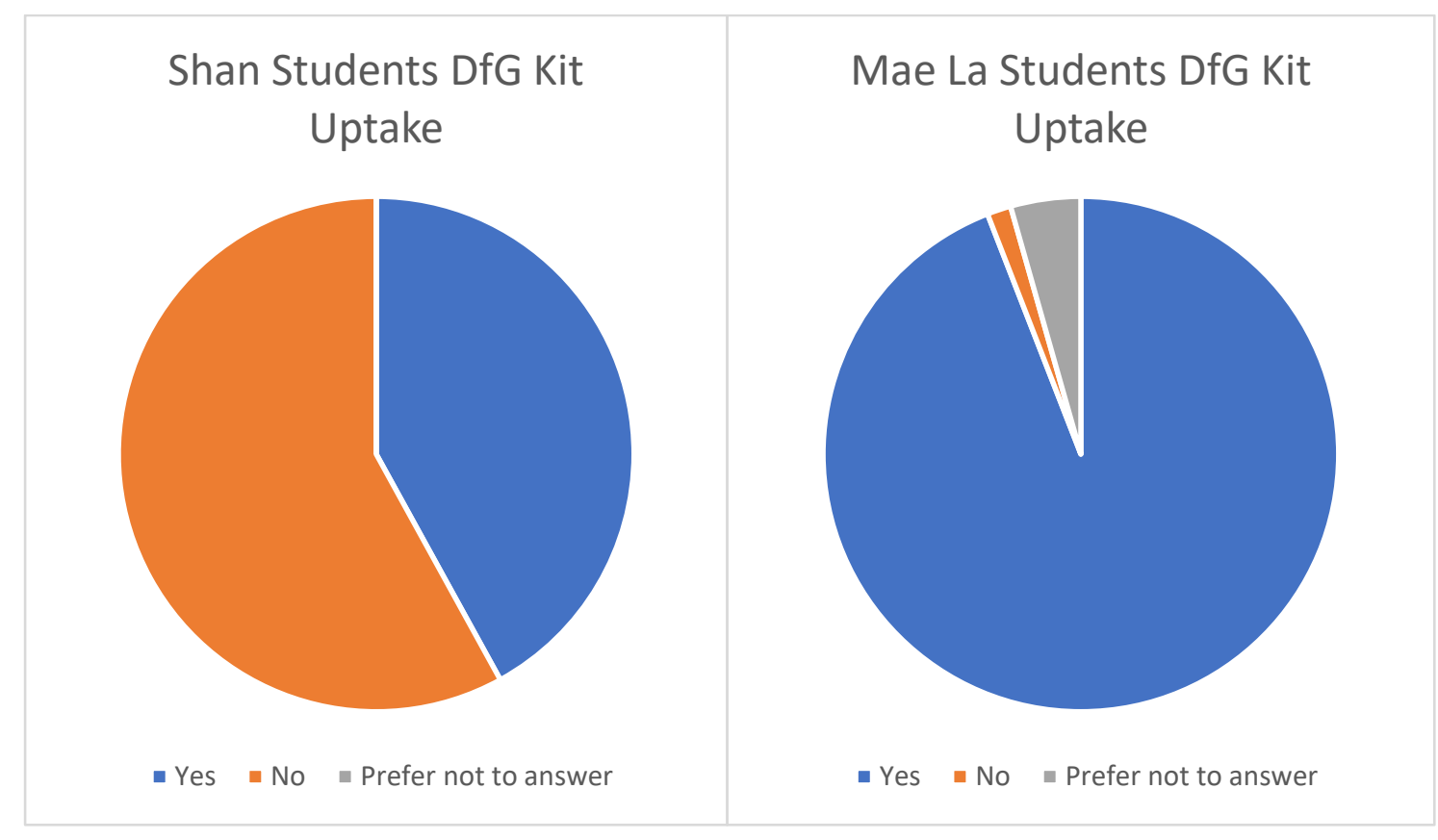

Figure 6.2: Shan students DfG uptake. Source: (T. Bardsley, 2019)

Figure 6.3: Mae La Students Kit uptake

I asked Partners staff if they knew of possible reasons for the difference between these outcomes. Their first suggestion was that the DfG programme was better established in Mae La camp. They said that this had allowed them to get to know the students there and visit several times with kits. The head of the Partners Health Department suggested that students' use of the kits may increase after they had time to get used to the idea of the new period product, like the students in Mae La had had: "Some products, you see it for the first time and think, oh, no, I don't really want to use it, and then maybe the third time you'll want to use it" (Ko Thu Lu student).

This suggestion corresponds to a study in the Middle East (Vanleeuvewen \& Torondel, 2018) where being "accustomed" or used to a product is a factor for acceptability of new menstrual products ( $\mathrm{p}$. 357). Based on these answers and the corresponding literature, the high uptake in Mae La may be associated with Partners' commitment to serving long-term in Mae La with this programme. It seems that staff and students have been able to build connections over this time (as explored in Chapter 5). 
Staff also suggested that the Shan students would have more difficulty drying the cloth in the DfG Kit because of the lower temperatures and higher levels of rain in their location (Partners staff, recorded discussion, 26 August).

\subsection{Mae La students: Confidence}

Overall, the Days for Girls Kit distribution has been impactful for the population surveyed. It was mentioned in interviews that most of the students have access to disposable menstrual pads and had previously used them, but there is a large majority who have reported switching to using the Days for Girls Kits. Most students said they preferred the Days for Girls Kits over disposables for varied reasons which were explained in the post-survey interviews.

One of the overarching themes among these reasons was 'confidence'. Mucherah \& Thomas (2017) argue that MHM relief can help women and girls to feel confident in their abilities to deal with their periods and to experience decreased levels of anxiety and shame that are part of many societies' views of menstruation. Accordingly, the survey results from Mae La showed that the DfG Kit helped 79.4\% of students felt more confident or comfortable when managing their menstruation.

In my conversation with Eh Moo, the concept of confidence in menstruation came across as one of the aims for the DfG programme:

Eh Moo: When they get the kits, they just hold it and they dare not to open it, they're shy! ... We tell them, "Only girls, no boys are in here, you can open it" and they just open it. But now they're used to it.

TB: Do you think that it [the programme] makes them more open about talking about it then?

Eh Moo: Yes. I think so. Before they're just so embarrassed.

Eh Moo spoke about 'embarrassment' of students during menstruation several times and seemed to think that the kits and the programme countered this embarrassment effectively, through the provision of products to prevent leakage and through encouraging students of the normalcy of periods. From my observations too, the brightly-coloured and patterned kits that were distributed in classrooms were received with joy and curiosity. After a brief moment of hesitancy, they would start to happily compare and examine the fabrics and each new part of the kit.

The following section will discuss some of the reasons, related to confidence, that students gave for their use of the DfG Kit and how it has affected their lives. 


\subsubsection{Comfort}

Participants in interviews said that they were confident wearing the DfG Kits out because they were comfortable to wear. $91.2 \%$ of the students in Mae La boarding homes answered that they found the kit comfortable to wear. This statistic surprised me, as in previous research with Shan students, participants identified the bulkiness of the shield and liners as a key reason for not using the kit (T. Bardsley, 2019). But some of the students I spoke to said they just needed some time to get used to the DfG pads (see above quote by Ko Thu Lu student). The students said that now, they find the DfG Kit more comfortable to wear than disposable pads. MM, one of the women who sew the pads agreed - she said that the DfG Kits are "not hot, but cool, and also soft"s.

Students said that compared to disposables, the DfG pads are less itchy, cooler to wear and feel comfortable because they are more secure:

Comparing to The Day for girls' kits and pads from shop, the kits are easier for me to use because the other pads are hot and move around. (Ko Thu Lu student)

As for me, the pads are hot and it can be broken or got apart accidently that the heavy blood can flow outside of pads. Therefore, the kits are comfortable for me to use. (Ko Thu Lu student).

Interestingly, some students said that despite the kits being the preferred pad, they did not always use their DfG Kits at home because the most comfortable option was to use nothing:

When I go to school, I will use it. But at home, I don't want to put anything there... I just feel more comfortable when I don't put on anything. Because when you put on something, covering it, it's something. So we just feel comfortable with nothing. (Htee Moo Traw student)

After the most comfortable option of wearing no menstrual products, the students felt most confident to go out using what they voted the most comfortable pad option, the DfG Kit. The preference for resuable pads above disposables is in line with the move that the MHM movement is making (towards the promotion and increasing provision of reusable pads and menstrual cups) because of difficulties with disposal, comfort in health and environmental issues (Vanleeuwen \& Torondel, 2018; Walkling \& Miedema, 2015).

\footnotetext{
${ }^{5}$ In my discussion with Partners staff, the Health Programme Coordinator shared that people from Burma often refer to their health and comfort in terms of temperature-levels. Heat can be considered harmful and balancing the body's temperature is key to maintaining health.
} 


\subsubsection{Ease of use}

Despite the lengthier process of assembling the pad and washing and drying it between uses, students generally said that the DfG Kit was easier to use than disposable alternatives.

We felt good because it is easy for us to go out when we put them on. (Ko Thu Lu student)

It is easy to use. Not too big, not too small. (IRC student)

Even of the washing process, $80.9 \%$ of the students in the survey said that the DfG Kit was easy to clean. They could each describe to me how they cleaned the kits in the laundry facilities at their schools and said that they felt confident with this process.

Eh Moo said that for the students, washing and drying the kits is easier than finding places to dispose of the used disposable menstrual pads in the refugee camp setting. Likewise, in other studies and NGO observations, it has been found that menstruators can have difficulty disposing disposable pads in places where there is limited waste infrastructure (Days for Girls International, 2019d, Sommer, 2012).

\subsubsection{Safety and health}

Students discussed aspects of safety and health when reviewing the DfG Kit compared to disposable pads. One student said that when she uses the DfG Kit, "My skirt or my pants are not dirty because I am more safe when I put on the DfG Kit. I am not afraid to go out because I am wearing it. It is safe, it will catch my blood." This student's emphasis on safety was discussed by other participants and highlights the security they associated with a larger pad. They mentioned that the way the pad stays secure on the underwear and the option to stack liners contributes to their confidence during menstruation - they have much less fear around blood leaking. This confirms the aims of the Days for Girls Kit design - to be customisable for the needs of the girl (Days for Girls International, 2019e).

As well as a sense of security, students talked of feeling safer in their health when they use the DfG Kit. Before my interviews with the Mae La students, Partners' Migrant Programme Coordinator told me of the health impacts of the kit, "Some people, they are allergic to use the pads. After they change to DfG, they don't have any problems." So I asked students whether they resonated with this. They didn't mention allergic reactions, but they did tell me:

When I use the disposable pad, I feel that my blood doesn't come a lot and it is itchy. So I feel it isn't comfortable and it makes the blood not come a lot. But the DfG one, that's fine. (Htee Moo Traw student)

We didn't like to use the pads from shop because sometimes the pads' date is expired and we feel itchy to use it. (Ko Thu Lu student)

Whether for not worrying about irritation from disposable pads or not worrying about leaks, students expressed that the DfG Kit helped them feel safer and more confident during menstruation. 


\subsection{Mae La students: Agency}

As I developed my interpretive analysis, another key theme that came to the fore was 'agency'. Omata (2017) defines agency within social science as "people's capacity to define their own choices, to devise strategies and to take initiatives to accomplish their objectives." (p. 144). From research in Lebanon and Jordan, Kikano \& Lizarralde (2019) wrote that refugee camps can be sites of agency and resilience "in which refugees negotiate, get self-organised, and adapt" (p. 32). This section will describe the ways students felt they gained and exercised agency through the use of the DfG Kit.

\subsubsection{Money}

One of the most common comments from students about the benefits of the DfG Kit was that it meant they didn't have to continually purchase disposable pads, thus saving money: "I was happy when I got it because it doesn't cost any money. This is for free and I can use it again and again" (Htee Moo Traw student).

Some participants said that there were inequalities of income between students; while some could use money sent from relatives to buy from stores that stocked disposable pads, many students could not afford them. In some cases, staff at the boarding homes bought disposable pads for these students. But the financial relief that came with the DfG Kit was evident:

\section{TB: What do you think has been the most significant impact for you?}

IRC student: I feel that is it nice to use the DfG Kit because I can use it again and again after washing it. And it doesn't cost any money to buy. For the pad, we can't buy, we don't have money to buy pads.

The students I spoke to enjoyed the reusable aspect of the DfG Kit because they no longer had to rely on insecure income to buy disposable pads - each student now had their own way to manage their menstruation, whether they had money sent from relatives or not.

\subsubsection{Mobility and participation}

Agency and mobility are inextricably linked. Chatter (2013) says that mobility is a form of capital that allows us more capacity to make choices and exert power. It is tied to the networks and power structures we are part of but is also reliant on material aspects of life (Hague \& Armstrong, 2010). Therefore, mobility is socially unequal. The concept of mobility is important for those in refugee camps, and especially Mae La where residents see themselves as trapped in many ways (Humanity \& Inclusion, n.d). While participation outside camp borders may be restricted, it is important to residents' sense of agency that they still have access to physical, social and spiritual spaces.

In the survey, $66.2 \%$ of students indicated that they were able to attend school more often now that they had their DfG Kit. This result would seem to confirm the link made by NGOs and some researchers 
between MHM and school attendance (Days for Girls International, 2019b; Girl Effect, 2008; Perczunska \& Preiss, 2014). However, I found out in interviews with students that many of them wouldn't miss school during menstruation when they were using disposable pads. In order to further understand impacts of the DfG Kit on mobility and participation, I asked students to take part in a 'mapping' exercise during our interviews. Students expressed diverse experiences through this activity.

Because most students had already been using disposable pads, some drew maps that demonstrated a small or zero difference in mobility (Figure 6.4). For example, this map by an IRC student showed that the only places she felt uncomfortable visiting during her period were the market and the sports ground. This student didn't 'tick' any locations on their map, showing that she still could feel restricted in participating in these places even when she had used the DfG Kit.
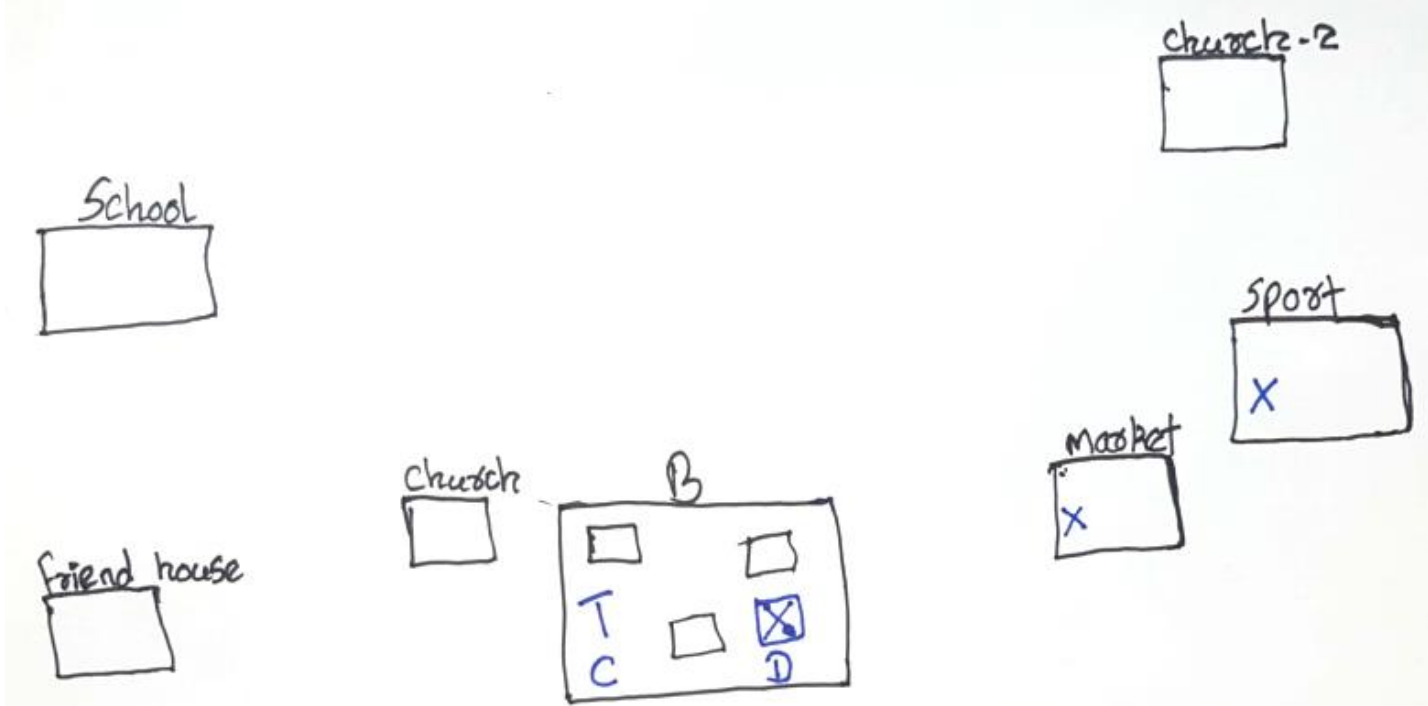

Figure 6.4: Map of places that this IRC boarding home student goes. Key: $\otimes=$ kit distribution site, $B=$ boarding home, $T=$ toilet where she can change her DfG Kit, C= where she can clean the kit, D= where she can dry the kit, $X=$ where she felt uncomfortable to visit during menstruation before receiving the DfG Kit

However, most students' maps showed a difference between students' subjective mobility (places where they felt confident to go) before and after receiving their DfG Kit. The map below (Figure 6.5) by a Ko Thu Lu student shows crosses by the shops, the school, the football ground, the church, her friends' house and the vegetable garden. These indicate that she felt restricted in visiting many every-day sites in her community during menstruation before she received her DfG Kit. The student used red 'H's (mistranslated from a 'tick') next to some crosses to show the places where she now felt more 
comfortable to go on her period - the shops, the school, the church and the football ground. She did not put an 'H' next to her friend's house or the vegetable garden. She did not specify why.
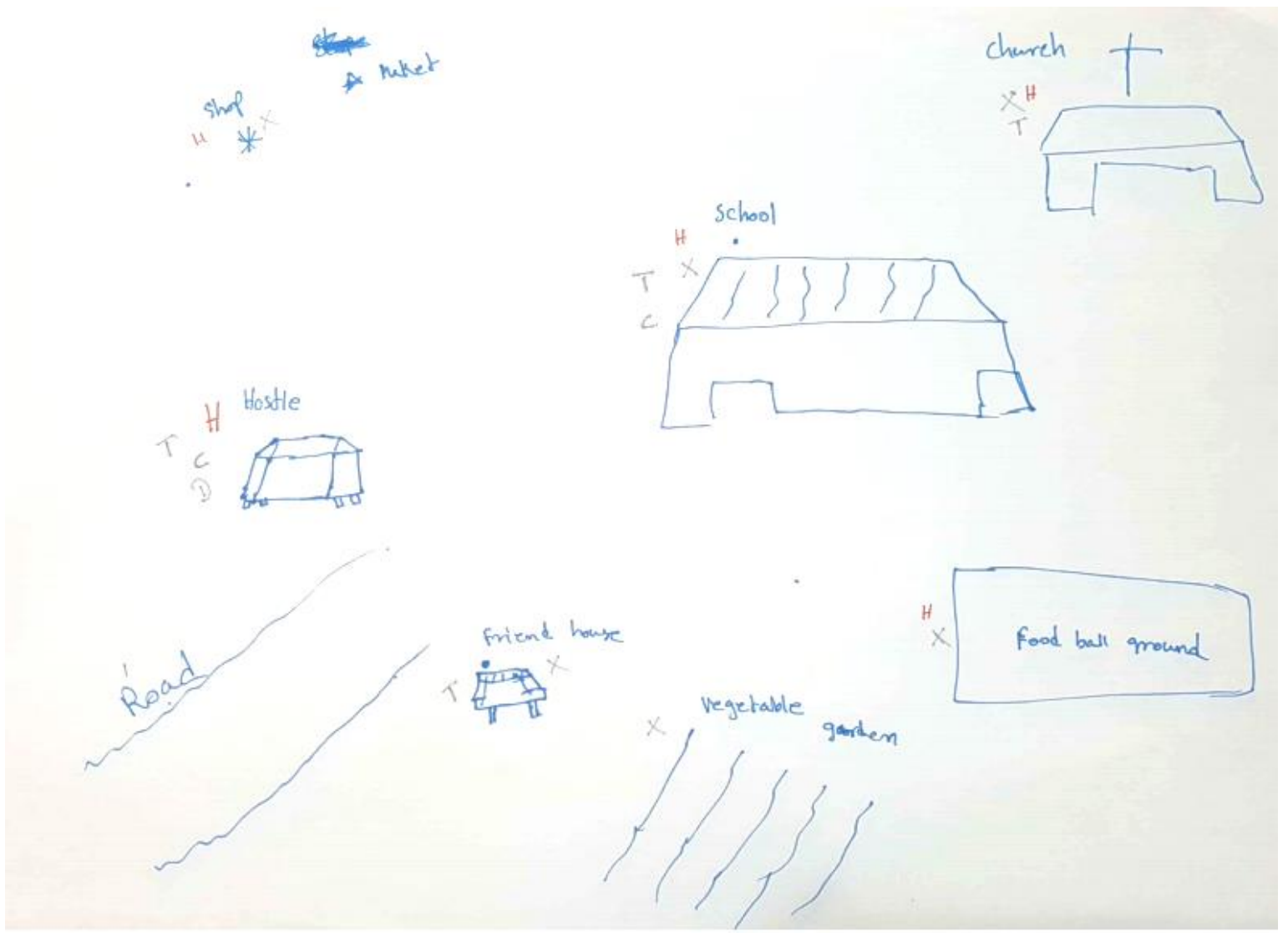

Fig 6.5: Map of places that this Ko Thu Lu boarding home student goes. Key: Hostle = boarding home, T= toilet where she can change her DfG Kit, $C=$ where she can clean the kit, $D=$ where she can dry the kit, $X=$ where she felt uncomfortable to visit during menstruation before receiving the DfG kit, $H=$ where she felt she could now go more comfortably after receiving the DfG kit

This student from Mission boarding home marked a significant difference that the kit had made in her life (Figure 6.6). She indicated that she had felt restricted going to the high school, another school, the football field and two churches during her period, but that now she felt less restricted to visit these places (shown by upside-down ticks: 


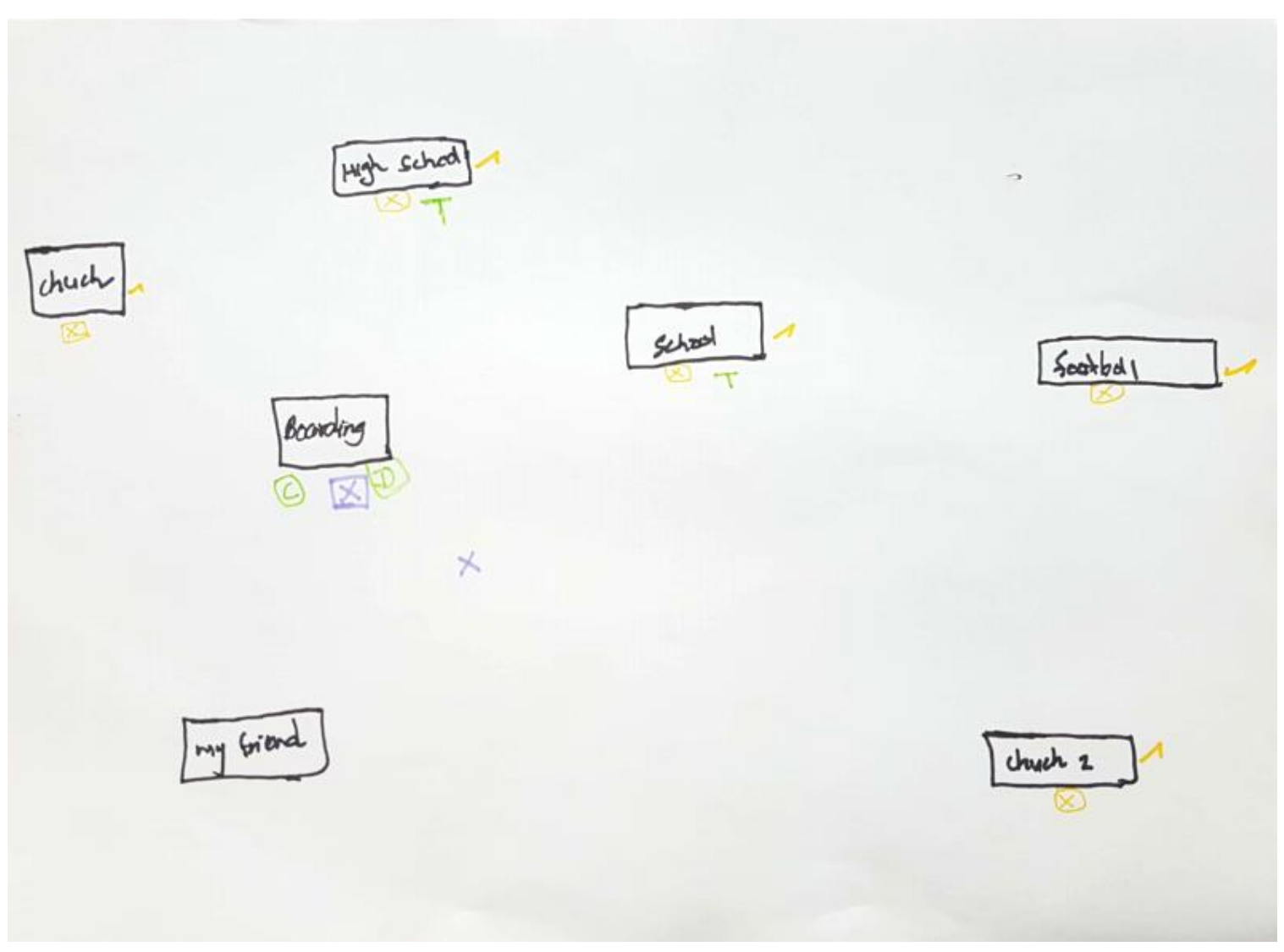

Figure 6.6: Map of places that this Mission boarding home student goes. Key: Purple $区=$ kit distribution site, T= toilet where she can change her DfG Kit, C= where she can clean the kit, D= where she can dry the kit, Yellow $X=$ where she felt uncomfortable to visit during menstruation before receiving the DfG kit, $\hat{\mathbf{N}}=$ where she felt she could now go more comfortably after receiving the DfG kit

Another symbol I asked students to make on their maps was a ' $T$ ' for locations of toilets where they feel comfortable to change their DfG Kit if they need to, a ' $\mathrm{C}$ ' for where they clean their kit and a ' $\mathrm{D}$ ' for where they dry it. Some students also marked the distribution site with a $囚$. Each student's map showed that they clean and dry their kits in the boarding homes. Some students marked that they felt they could change the kits at their schools, churches or friends houses but in interviews, they said that they usually only needed to change at their boarding homes. They said they would go home (to the boarding home) for lunch anyway and didn't need to change them more frequently than that. Unlike reports in other countries (Sommer et al., 2017; Hennegan et al., 2017), these students did not express problems with lack of facilities for changing/cleaning pads.

In interviews with these students, we discussed the maps they had made in terms of participation. They particularly talked about their new confidence taking part in activities such as church services and football games. One Ko Thu $\mathrm{Lu}$ student said: "I can go to church and go out among of people comfortably without worrying that the blood will appear outside anymore." Some students also expressed agency in the way they used their kit: "If I have heavy blood, I used two or three layers of 
cloth to put it in." (Ko Thu Lu student). This correlates with findings from three camps in Tanzania, where participants said that the reusable pads lowered the chances of blood leaks onto clothing, enhancing mobility during daily community activities (Clatworthy et al., 2018).

These effects on mobility and participation are perhaps one of the strongest links to Partners' vision of 'Free, full lives for children affected by conflict and oppression.' The maps drawn by students showed an increased ability to choose what activities they participated in, rather than be restricted by worry of leaking during their period. One Ko Thu Lu student said, "We can go wherever freely after receiving The Days for Girls' kits."

\subsection{Migrant community women}

Part of Partners' vision for the Days for Girls programme is to "continue to be able to give women work" (Sarah Kahn, 2019). In Mae Sot, the programme is supporting working migrant women in two ways: the women who sew the DfG Kits are offered stable, fairly paid work; and women from Burma can go to work with the confidence and agency of a Days for Girls Kit. This section will discuss the ways some migrant women's lives are affected by using a DfG Kit.

Only two migrant women who had received Days for Girls Kits as part of a distribution were interviewed as part of this study so this section is not meant to be representative. The two women interviewed worked six or seven days a week in a sweatshirt making factory. They both said that they enjoyed using the Days for Girls Kits and that the biggest impact of the programme for them was a release of financial pressure.

\subsubsection{Confidence}

There were some answers from our interviews that related to women's increased comfort and confidence when wearing their DfG Kits. Like the students, these comments revolved around security:

After I received DfG Kit, it's more comfortable, I can go wherever I want to, I can do whatever I want to. I don't have to be afraid about my blood, like, people seeing my blood. (Migrant Community Woman).

I feel, like for the DfG Kit you can get a thick one, I have more confidence. But to get a pad from the shop it's very thin, and I think, 'ooh, it's gonna leak. (Migrant Community Woman).

While the women said they still came to work during menstruation when they were using disposable pads (this wasn't a choice for them), they expressed that they now felt more confident during work and their daily routines because they found the DfG Kit less likely to leak.

\subsubsection{Agency}

For the women I interviewed, the most important impact of the DfG Kit was that they could save money by not buying disposable kits. From my enquiries with Partners staff, the financial effects can be 
significant for these women. I asked Eh Moo to help me estimate how much women are likely to save: In Mae Sot, a small pack of Kotex costs approximately 20 baht. Eh Moo said that on average, women use two to three of these packs per month. This means that over two years, women can save around 1,200 baht (NZ\$61). Considering women in Mae Sot factories earn on average 300 baht/day (NZ\$15) and women in the Mae Sot farming community earn about 150 baht/day (NZ\$7.70), this saving is important to them, as they reported.

The women I interviewed were eager to share this impact with me, often speaking louder and more excitedly when it came to this topic:

MCW: It's very useful and I am very thankful because, after I got DfG Kit I doesn't need to buy pad from the store anymore.

TB: Yeah-are they expensive?

MCW: Yeah, if you get one pack, it's not expensive, but when you get more, it's getting more expensive, more expensive.

They both said that the release of a financial burden allowed them to spend on other essential costs for their families:

\section{TB: What do you use the money for instead?}

MCW: Just use for the housing, and for the family. [...] The money that I can save, I use for the other things, it's useful and helpful.

While the disposable pads were not a main cost for these women's families, the release of this monthly expense was significant to them. In Mae Sot, women are often the sole earners in their families because many employers do not like to hire men as they think they will be unreliable (due to issues of alcoholism and related cycles) (Gaitan et al., 2019). So, the reusable DfG Kit allows women with little disposable income some more choice on where to spend their money.

\subsubsection{Mobility?}

In many of my interviews, I wanted to understand the impact of the DfG Kits on mobility. From previous reviews of MHM literature, this was one of the most significant impacts for women and girls (Clatworthy et al., 2018; Ritu, 2017; Sommer et al., 2015). But each community's situation is different, and my assumption was challenged as I interviewed migrant women.

At the end of each of my interviews, I asked participants a 'Dreaming' question informed by Appreciative Inquiry. For those that had received DfG Kits, I asked the question, "If you had unlimited resources, what would be your ideal way of dealing with your period?". I was expecting that participants might talk about other MHM measures they may have seen or a means to not having a period. But I was 
surprised by one woman's response. She laughed as she replied: "If I had unlimited resources, when I had a period, I would stay home, I would not go out. Even like come to work, I would not come to work." For this woman, increased mobility and increased ability to get to work was not what she wanted for a 'free, full life'. She was tired from working 50-60 hours per week and a chance to rest was what she dreamed of. It is important to acknowledge that while the DfG Kit solved some issues for menstruators in this study, their daily-life concerns went far beyond covering up during menstruation. They connected to enduring feminist concerns associated with unequal gender division of labour and women's triple burden (reproductive/domestic work, productive/income work and community management work) (Moser, 1989). For the migrant women I interviewed in the sweater factory, the biggest impact of the DfG Kit was release of financial pressure, followed by not having to worry about leaking during menstruation on top of the other 'burdens' in their lives.

\subsection{Women who sew DfG pads}

It is not only the recipients of the DfG Kit that are impacted by the programme. One of its core aims is to provide steady, skill-building employment for those from Burma who have already crossed into Burma and are trying to build a new life. Four migrant women who are employed by Partners to sew Days for Girls Kits were interviewed as part of this study. The participants took the 'appreciative' questions gladly and expressed strong positive feelings towards their work:

TB: What is the best thing about your work here? What is your favourite thing?

MM: It's all my favourite. Every hour is my favourite!

The responses generally also fitted into the categories of 'confidence' and 'agency':

\subsubsection{Confidence}

The women I interviewed seemed proud of the work they did at Partners. MKO told me that other people in her life are impressed with her skills, "Sometimes people say, "oh, it's difficult, DfG! It's a difficult one to sew!'”. MKO knew that the work she did required a high level of skill and seemed happy when people recognised that. Likewise, NSL recounted the journey of skill-learning and what that means for her today: "Last year, I just learnt. And right now, I can do almost everything. I can sew a bag or dress. The things that I learnt from here, I can do everything." NSL's repetition of "I can do [almost] everything" is a testament to the confidence she has grown and the satisfaction she feels in her abilities. For her, these skills mean she can contribute to the community she came from back in Burma.

There was also a sense of purpose in the words of these women. Some of them talked about their capacity to teach other women in Mae Sot (who could then sometimes teach other women), now that they had learnt skills at DfG. 
MKO: I also train other people. I can train other people in Mae Sot. [...] I train the trainer and they train other people.

TB: What is important to you about training other people?

MKO: The most important thing in training other people, is to see if they really understand. If they don't understand, it really affects me.

MKO's statement about being affected if her trainees understand her teaching or not shows that she values doing her job right. This correlates with statements from other women about being careful to sew the kits well, to honour the recipients with a good-quality product. For example from NKP: "I value everything. Because the clothes we make should be good for other people, so we put in every effort. Whatever we can do."

Employment for refugees and migrants has been shown to be meaningful not only for incomegeneration, but for the chance to contribute to communities: "[My work is a] kind of present to society; something from me. It's not only [the] money; it's a self-respect and dignity part of it..."” (Eritrean former refugee) (Searle et al., 2012, p. 65). The responses from the women employed to sew the DfG Kits shows that their employment can also be an opportunity to serve refugee and migrant women.

\subsubsection{Agency}

The women showed agency in two ways as a result of the DfG employment: through the money earned and the skills taught. NSL, especially, spoke of the importance of earning an income for life as a migrant from Burma in Mae Sot:

I feel so happy because I get skills and also I get paid. If I stay here, I also need legal documents to stay here, so I need pay to pay for the legal documents. And I thank Pipi Sarah for training me, because my family cannot support me so it's a really good opportunity for me.

As well as money for daily life and immigration documentation, the skills learnt through the programme are also a means for agency. I asked the women what they most value about being part of the programme. In response, three out of the four women shared their excitement for past or potential future opportunities to share their sewing and DfG knowledge with other migrant women and their home communities in Burma.

TB: What is your favourite part about working for Partners?

NSL: Everything in my work with Partners. But my most favourite thing is getting the skills provided from Partners and Pipi. [...] After I learnt here, I can go to share in my village. 


\subsection{Conclusion}

Partners' Days for Girls programme has had a meaningful impact on menstrual health management in the communities where it operates in Mae Sot and Mae La. The 94.1\% student uptake shows that the kits are a preferable method of period management for most students. While this research does not claim a clear causation between the DfG Kits and school attendance (as similarly suggested in Bobel (2019) and Grant, Lloyd \& Mensch (2013)), each of the students interviewed said they feel that the kits help them to feel more confident and comfortable and many now feel safer going to school and other community activities during menstruation. Within the insecurity of Mae La camp, measures that increase feelings of safety and confidence can be impactful for the every-day life of refugees. Further, those who feel they can participate in more community activities can experience benefits to their psychosocial health (Maclean, 2014).

For many women and girls in this study, the reduction in financial pressure caused by buying disposable pads every month was also important. And for the women who sew the DfG Kits, the work provided a sense of purpose and a source of income and skill-education that made them feel more agency. As mentioned, greater security in employment can be essential for improving the resettlement experiences of migrants and refugees - through both the ability to provide for themselves and their families, and through the opportunity to contribute to their host society (Searle, 2012).

The DfG Kit outcomes covered in this chapter are a result of the efforts of women and Partners staff that were a part of the production chain of the DfG programme as well as the choices made by participants for their own menstrual health. While the findings discussed so far have been associated with the DfG Kit, the next chapter (Chapter 7) will address outcomes to do with knowledge and education. And while the outcomes so far have also been 'appreciative', barriers to such outcomes and areas for the programme to develop will be discussed in Chapter 8 . 


\section{Chapter 7 - Knowledge}

\subsection{Introduction}

Education is a key part of the Partners Days for Girls programme. Kit distributions are conducted with menstrual health training including: information on period management, the menstrual cycle, female anatomy, and even self-defence and trafficking awareness. But there is also a wider aim of the programme as a whole - to combat shame surrounding menstruation for women and girls.

Following Appreciative Inquiry, this chapter discusses the strengths of the knowledge-sharing that is part of the DfG programme, reviewing what students remembered from the training. There is a discussion of areas for future teaching topics, focusing primarily on what students still want to learn about menstruation. Finally, there is also be a discussion on menstruation stigma which is informed by critical feminist theory. I will ask whether the DfG programme is working against or contributing to this type of stigma.

\subsection{The DfG Health Education - Effects}

Days for Girls International has a 'health education' course that is taught alongside kit distributions. Partners staff and volunteers who carry out distributions are asked to take part in a 6-8 hour 'Ambassador for Women's Health' online course that trains them to teach the material from the Days for Girls flip-chart (Figure 7.1). The chart contains clear, brightly coloured images and diagrams and includes information in English or translated into the language of the programme location. So far, Partners has booklets translated into Burmese, Karen and Thai. 


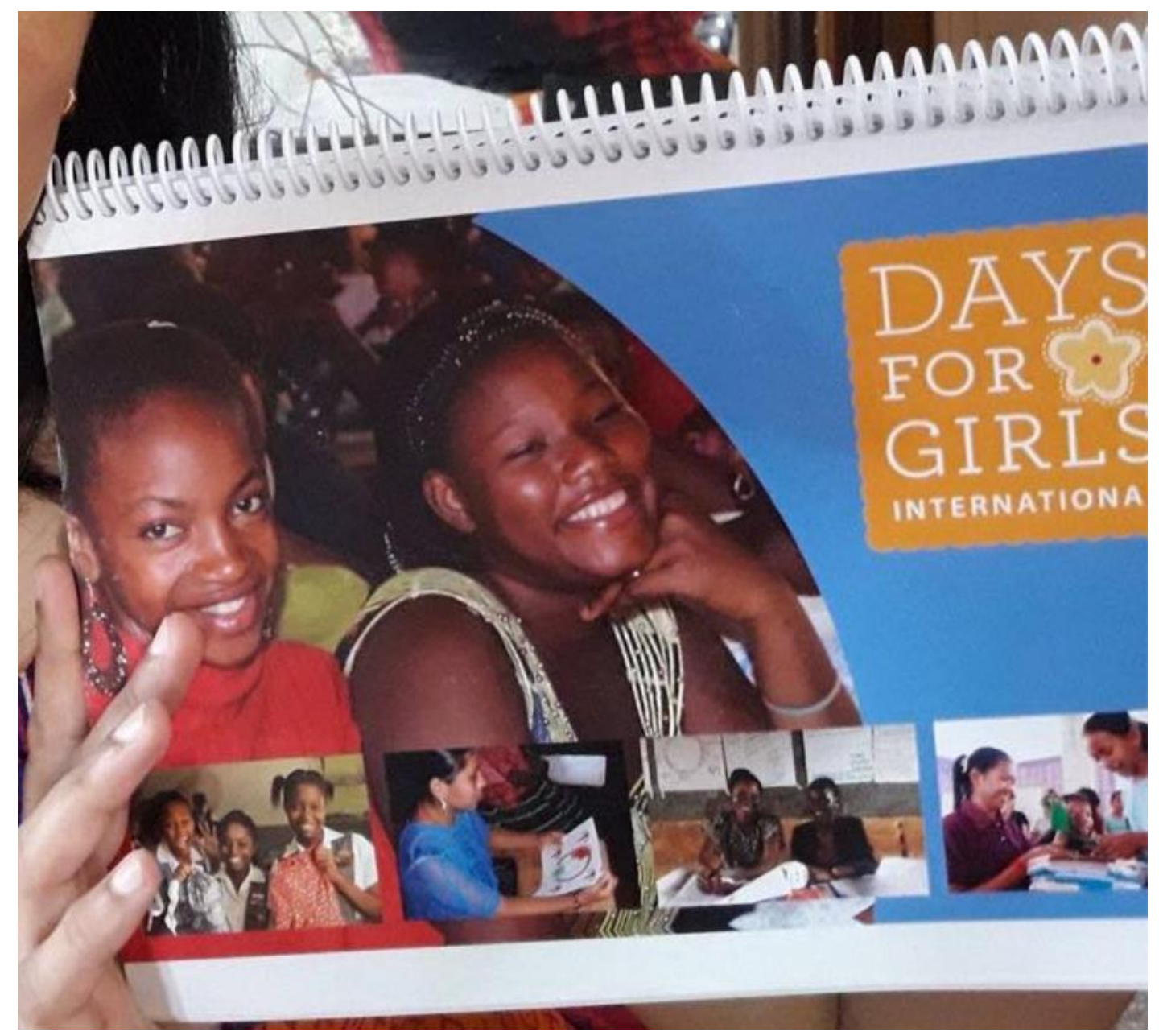

Figure 7.1: The Days for Girls Flipchart (image adapted from Sew for a Girl Canberra, 2017).

\subsubsection{What was taught}

The education begins by overviewing a wide range of sexual health topics: puberty, the female and male reproductive systems (eg Figure 7.2), how to prevent infections, intercourse, and STIs. It then looks at the menstrual cycle. It teaches the phases of the cycle and how there can be variation between women. There is an emphasis on seeing menstruation positively, with phrases such as "Menstruation is a natural and important process. It is the sign of a healthy girl or woman" (Days for Girls International, 2016, p. 2). 

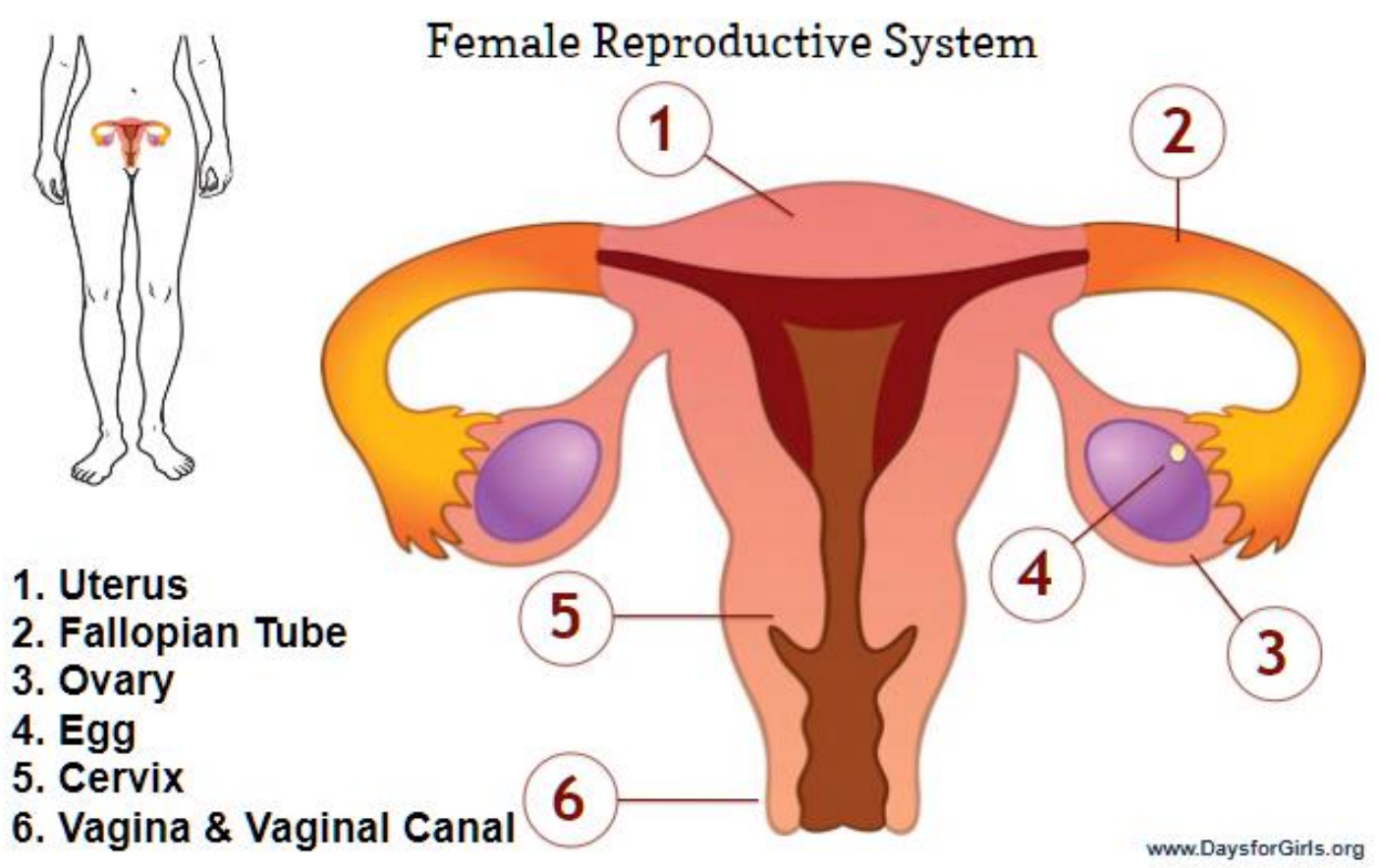

1. Uterus

2. Fallopian Tube

3. Ovary

4. Egg

5. Cervix

6. Vagina \& Vaginal Canal

Figure 7.2: Days for Girls Flip Chart Pg 7 (Days for Girls International, 2016)

The teaching talks a little about contraception, mostly regarding how there are times in your menstrual cycle when you are more or less likely to be pregnant. From my impressions, Partners focuses more on using cycle tracking for contraception than is required in the Days for Girls International guidelines. Sarah and other staff members talked about the importance they placed on this topic - that they weren't aware of any other formal sexual health training for the girls and women they work with, and it that they knew of young girls becoming pregnant without the knowledge of how to avoid pregnancy. They believed that this knowledge about fertility and the menstrual cycle could give women and girls more agency over their bodies.

Other topics included in the training covered how to wash and dry the kits and hygiene - with a page titled, 'Washing Hands Saves Lives'. The programme then tackles two large topics in the last few pages of the flip-chart - trafficking and self-defence (Figure 7.3). The trafficking information includes a series of ways that traffickers try to 'trick' victims. The self-defence information includes a discussion about sexual abuse - what it is and some ways to seek help. These topics are very complex to be covering in such a short time, especially considering that Days for Girls International envisages that several of the audience at a training may be struggling with these issues (Days for Girls International, 2016). Academic studies of abuse and sexual prevention education show that teachers should carefully present these topics, leaving enough time to ensure the information is culturally relevant, well-understood and sensitive to students who have already experienced trauma (Alfath, Firman \& Syahnair, 2018; CollinVézina, 2013). The Days for Girls manual encourages instructors to cater the training to the participants' context, including the cultural context that they are a part of and to speak about the topics sensitively. 


\section{SELF DEFENSE -- Stay SAFE}

SURVIVE - Because YOU matter.

ALWAYS be AWARE of what is around you. Walk strong.

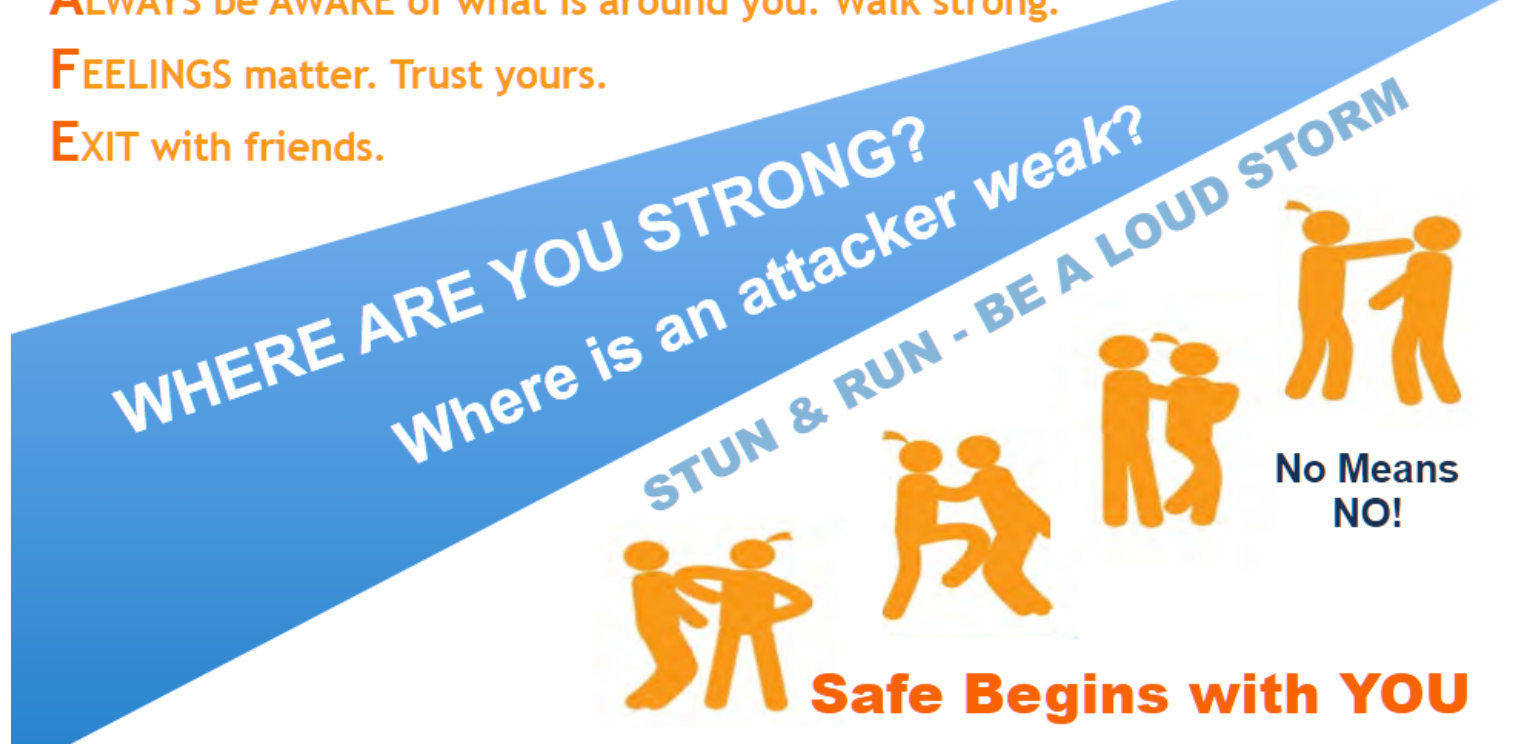

Figure 7.3: Days for Girls Flip Chart Pg 33 (Days for Girls International)

\subsubsection{What information was retained?}

When I asked the students what they remembered from the DfG training (that had taken place between four and fifteen months previous, depending on the boarding home), most of their immediate answers were centred around the menstrual cycle and how to use the DfG Kit:

They need to dry under the sun, not hide in the shade. (IRC student)

We just remember that every girl will have their period once a month, so if it comes like, on the 23rd, so in the next month it will come on the 23rd. (Htee Moo Traw student)

Apart from this question, I also recorded 'retention' through the ways students discussed their process of looking after the kits. All students told me that they washed and dried the kits in the way that they had been taught. "We wash and dry them in the dormitory. There is enough space to hang them. In the rainy season, we dry them outside first. If the water is too much, we dry them underneath the house" (Htee Moo Traw student).

Some students said they recalled learning about wider issues including puberty and the stages of the menstrual cycle: "There are some photos about adult women growing before they are in period, and when women can be on period or can have baby that Eh Moor had shown us" (Ko Thu Lu student). Students also said that they had a chance to ask questions during the training and that they remembered some of those answers: "We asked why our back is really hurt and have abdominal cramps before the 
menstruation take place. Eh Moo replied that it is the sign for women to be on their period" (Ko Thu Lu student).

After the general question ("What did you remember learning?") I asked about specific aspects of the education, to see if it would help students' memories. This produced varied answers. Some said they remembered learning about self-defence but not contraception, and others said the opposite.

The Mae La students remembered fewer topics in general than in the previous study of Shan students (Bardsley, 2019). The students talked less about intercourse, STIs and contraception. The Shan students' stronger memory may be because they had a longer, more in-depth question and answer session with the instructors, as they reported. When I presented this information to Sarah, she confirmed this idea. She said that in some early distributions in Mae La, "They gave a scaled down presentation due to time constraints and the fact that the DFG Menstrual Health training had not been completed by the person presenting." She said that the women presenting are now all trained in the Ambassador for Women's Health course.

Overall, it appeared that the retention focused mostly on how to use the Days for Girls Kit, and how periods worked. A study in Malaysia of students' sexual-health education recollection showed similar results. Students in this study said they remembered more information about human anatomy and biological functions, as opposed to social and relational issues (Mokhtar et al., 2013). Doyle (2008) suggests that students need constant exposure and reminders of the education they have received. In the same way, it could be that information relating to the DfG Kit was retained because it relates to a physical object that they use every month, and information about the menstrual cycle was retained because they go through this cycle every month.

\subsubsection{What else students would like to know}

In the interviews, students were asked if there was any additional information they thought should be included in future training. This was based on Appreciative Inquiry 'Dreaming' stage of inquiry (Stavros at al., 2015). Questions asked what students would hope to know about in the future, or what they thought younger students would benefit from learning in future trainings. At every boarding home, students replied with questions they had been curious about. The following questions arose from students in this discussion:

1. Why do we have thick blood [clots]?

2. Why do we have pain during our period?

3. What food can we eat during our period? What food should we avoid?

4. Can we wash our hair during our period?

5. What else should we avoid during our period? (For example, exercise). 
This question was asked at the end of our interview and students seemed to be much more comfortable chatting to me by this point. They were open about their menstruation queries - whether medical/science-based questions ( 1 and 2 ) or about their understanding of how to keep healthy during menstruation (Questions 3-5). The question of which foods can be eaten during their period was asked by students at three out of the four boarding homes. Sometimes it was accompanied by comments on what other people did or believed in their communities, “for some people, they can't eat spicy food and left-over rice" (Ko Thu Lu student). It seemed that students wanted to understand the perspective of the DfG programme on these issues, because they had found answers to their other questions from this source.

At every boarding home, students asked whether it was okay to wash their hair during menstruation. They said they had been told by their mothers and grandmothers that it could be detrimental to their health. For the students, this was a serious matter and was associated with fear for health. They spoke about the understandings they had gained about this from others:

From my experience, I have seen people who has a period and wash her hair and then headache too bad and then die... They were maybe 16. (IRC student)

We heard from the elder people saying about washing hair is not good for girls when being on period. They said being on period is like the time when girls' blood is not normal like ordinary blood and it is easy to get pressure in menstrual cycle, so it is not good to wash hair when we are on period. There were some girls who died because of washing hair during menstruation came. On this incident, I wanted to examine and sometimes I did wash my hair without realizing that it would hurt me. On my action, some people said you wouldn't feel anything in your young age yet, but you would face health problem when you would be in old age. Thus, I didn't usually wash my hair on menstrual period. (Ko Thu Lu student)

And Eh Moo spoke from her own experiences:

Different people have different opinions on menstruation. They said washing hair during menstrual period would make you to be clear and felt fresh. For me, if I washed hair when being on period, I felt dizzy and tired...

I asked Eh Moo if she knew how these health concerns came about. She said that this was what Karen people believed, "based on their experiences." She said that during menstruation, Karen women are concerned about their health, "especially the Karen from the jungle - they really take care of themselves because if something happened in period, it's not easy to take them to clinic or hospital." She wondered if the avoidance of washing hair was to do with staying warm so they didn't get sick and need medical attention. Similar practices have been reported from women's experiences in other cultures: In rural Fiji, girls reported that they do not swim or wet their hair during menstruation, because then "they would not 
menstruate properly" (Mohamed et al., 2018). Like the participants I interviewed, many of these girls viewed the restriction as positive, "believing it would keep them in good health" (p. 12).

On this issue, and the issue of what foods to avoid during menstruation, students faced tension between the ways of knowing they had been taught growing up and a new set of menstrual health knowledge they received through the DfG programme. This tension and the politics of knowledge involved will be discussed further in the next chapter.

\subsection{Stigma and dignity}

Two of the key aims for both Days for Girls International and the Partners DfG programme are to: 1) reduce stigma surrounding menstruation, and 2) help women and girls feel more dignity during menstruation. This section will discuss the ways the Partners DfG programme does these two things.

\subsubsection{Menstrual stigma}

In my research, I wanted to understand some of the public perceptions of menstruation and feelings toward talking about menstruation within Karen culture. From my discussions with some Partners staff, I got the impression of a low level of openness about menstruation in Karen culture, and for some, a high level of shame:

They don't know why they have menstruation, and when they don't know that, they think well this is a bad thing, this is a dirty thing [...] There's so much fear around whatever they've been taught. (Sarah)

In our Karen culture we are afraid to tell our Parents because we shy. [...] They're afraid to talk about that, you know. They're shy. And so, it's kind of, it's kind of challenging for us also because they never talk about that, and even in the family in our Karen culture in the family. Only the girls would talk to their mother only and not with their father or with their brothers. (Head of Education Department, Partners)

In my interviews, this was partially confirmed as many students were shy at first to talk to me about menstruation. Some of the older students said they were open about the topic to an extent: "It is fine to talk and discuss about it to every girl, but we don't talk about it to boys" (Ko Thu Lu student). And the migrant community women I talked to were very open. When I asked them about how they felt about the programme, they gave practical answers that brushed off ideas of menstrual taboo/embarrassment:

MCW: It's okay to do the training, because like, it's a part of health, you know. Like no one can avoid it.

TB: Is there any embarrassment around using it [The DfG Kit]? 
MCW: It's okay. A lot of people get it here, it's not a problem. Almost everyone got it.

The difference in response from women of different ages is corroborated by the Migrant Programme Coordinator: "For older ones, not teenagers, they are really open. They talk about DfG, their bodies. But for the teenagers, they will be really shy to speak it out." Studies by Sanyal \& Ray (2008) and Aflaq \& Humaira (2012) show that age-increase is linked with the regularity of periods, and shorter periods (as well as longer experience managing periods) which could contribute to more positive attitudes towards menstruation.

It is impossible to generalise the views and attitudes of an entire culture on a subject. And it was not possible for me, in my limited understanding, to draw a single conclusion about the menstrual stigma and taboo for migrants and refugees from Burma. But these extracts from participant conversations show that there are a variety of levels of openness when it comes to menstruation within participants. There is also a possible effect of age on menstruation attitude (Sanyal \& Ray, 2008).

\subsubsection{How the programme has helped to combat menstrual stigma}

In line with its aim, the Days for Girls International health education is overtly positive about menstruation. The flip-chart associates menstruation with good health and beauty:

Menstruation Matters. Menstruation is a natural part of being a woman and a sign of good health. [...] All women are beautiful and powerful naturally. That means that our bodies and menstruation, also called menses and periods, are beautiful too! (Days for Girls International, 2016, p. 2)

Perhaps more naturally, the way that Eh Moo talks about her teaching is encouraging and positive. She has said that this makes them more comfortable with their menstruation and their DfG Kits: "We tell them only girls, no boys are in here, you can open it and they just open in. But now they're used to it."

While I did not ask students directly, they generally gave the impression that the menstruation education made them feel more confident. When I asked them how they felt immediately after the training and distributions, every student said either "good" or "happy". Many of the students talked gladly of the training involved and most said that they learnt new information.

Shame and stigma are social phenomena (Dorahy \& Clearwater, 2012; Walkup, 2015) and research shows that they can be reversed or mitigated socially. Group settings that facilitate discussion about stigmatised topics have been found to be particularly effective (Adams \& Robinson, 2001; Kinsman, 1997). Having a large group of menstruators together in a room, sharing about their experiences, can decrease feelings of isolation in menstruation and increase feelings of openness and solidarity for women. Even statements of what students learnt show that they acknowledged that menstruation affects many of students' peers. 


\subsubsection{The issue of 'dignity'}

The discourse shared by several MHM organisations, especially Days for Girls, is that "MHM is a matter of dignity" (Bobel, 2019; Days for Girls, 2017; Dignity, 2019; Partners, 2019c). The concept of dignity as linked with 'normalcy' is said to lend stability especially to those in humanitarian situations (Oka, 2014). The idea that the DfG Kit could be a product that brings dignity is suggested in some of my interviews with participants. Several of the students expressed the positive feelings they felt at receiving their own DfG Kit. Sarah also referenced the 'dignity' effect more than once during our interview. She told me of a report from one of the distributors in another country where a woman who had received the kit said, "Now I can have dignity".

But the discourse within MHM that links dignity with 'value' and 'self-respect' is critiqued by Bobel (2019). She writes that framing MHM in terms of dignity does not reduce menstrual stigma. Instead, it focuses on "managing the menstrual body so that no evidence of menstruation is detected" (p. 217). The theory is that the shame associated with menstruation is not reduced, but rather that through menstrual products, menstruators now have a way to hide the blood that causes shame. Bobel suggests that this is part of and helps to perpetuate the 'culture of concealment' and 'menstrual mandate' of needing to silence menstruation (see Chapter 2). The question being asked is: Should menstruator dignity hinge on access to a menstrual product? If dignity is inherent and intrinsic to every person (Beitz, 2013, United Nations, 2019), what is the implication when MHM brands itself as 'giving dignity'? Bobel does not suggest that MHM organisations should discontinue distributing products- "we must listen to menstruators and meet their present needs...” (p. 239). But she does suggest that MHM encourages women to recognise their dignity because it is inherent in them, not because they have a way to stop menstruation leakage. Furthermore, she proposes 'menstrual literacy' as the real solution to menstrual stigma. She encourages a teaching programme where girls and women "learn to "read" their bodies, engaging them with curiosity, confidence, and appreciation." (p. 315).

The knowledge that Partners shares through their training programme leads towards the type of 'menstrual literacy' that Bobel describes. There is in-depth teaching about the menstrual cycle and discussion of women's and girls' bodies as complex and varied, and bodies to be respected. Despite students not remembering every aspect of the teaching, every student interviewed said they learnt something new at the training. Furthermore, all students said that this was the only formal training they had received on menstrual and sexual health.

I believe that the strength of the Partners MHM programme lies not in the DfG Kit's 'covering up' of period blood or in the kits 'giving dignity'. Indeed, the kit does provide a feeling of security for girls living in a world where their menstrual blood leaking would be embarrassing. But more importantly, its brightly coloured and carefully sewn kit brings joy to girls and women during menstruation. It connects the women who receive the kits and the women who sew them who are paid a fair wage and find purpose 
in their jobs. And it teaches girls and women about the intricacies of their bodies and encourages them to ask questions.

\subsection{Conclusion}

Education forms a vital role in the Partners Days for Girls programme. It aims to use education to combat shame and increase confidence surrounding menstruation for women and girls. The course teaches on a wide range of sexual health topics to help its participants to read and have more agency over their bodies - for example teaching when in their cycle women are more and less fertile.

This chapter has examined the effects of the DfG programme on women and girls' knowledge of MHM. The surveys and interviews showed that the recipients of the kit took on much of the teaching about the menstrual cycle, the DfG Kits and puberty and some of the teaching about contraception, STIs and selfdefence. In keeping with Appreciative Inquiry Dreaming, ("What would you like to happen in the future?"), students hoped that future teaching would answer further questions about menstrual pain, blood clots and practices around food and hygiene that are recommended for menstruating women. I have suggested that this education would need to take into account cultural beliefs and the experiences of the women and girls they work with - not discounting the knowledge they have built but offering opportunities for open, respectful discussion and new learning.

I have also examined perceptions of menstruation in this chapter and the ways that women felt the programme reduced menstrual stigma. From my research, I saw that stigma was reduced through opening up menstrual literacy conversations, especially for the younger girls who felt embarrassment around the topic. There is further opportunity for Partners and other MHM providers to reduce menstrual stigma through education and open discourse that focuses more on menstrual literacy than dignity. 


\section{Chapter 8 - Analysis of the Partners MHM Programme}

\subsection{Introduction}

Having discussed the process of kit production and the effects of the programme for participants, I now turn to a more in-depth analysis of the Partners MHM programme. This chapter evaluates, through the participants' views, the key strengths of the DfG programme in terms of improving confidence and agency, as well as the challenges it faces. This section draws on the collective knowledge and experience that are held in the group of participants who live and breathe the programme (Reed, 2007). It then turns to an analysis of power and knowledge within the structures of the programme using feminist critique. Drawing on the themes of agency and confidence discussed in previous chapters, I begin to suggest ways that the DfG programme could move into the future. Through this chapter I also ask how these lessons could inform wider understandings of MHM in development.

\subsection{Strengths}

For those who had received the DfG Kits, the programme's strengths were its effects on agency and confidence. This section will cover further strengths of the programme through the perspectives of those who work within it. Many of these quotes were in answer to the question, "What do you most value about the programme/working in the programme?".

For the women who worked in the workshop sewing pads, answers to this question varied. As mentioned in Chapter 6, participants mentioned the sense of purpose, agency and confidence they felt in the creation of the DfG Kits for other people as well as the opportunity to build skills:

\section{TB: What are the things you most value about your work?}

MKO: Everything at work is valuable. Because it's the skills I can learn here. Making something that people can use, it's very helpful for other people, it's very nice for me. At the same time, I can earn money to support my family.

For the Migrant Programme Coordinator, one of the strengths was the quality of the DfG Kits. Several of the staff and women who sewed echoed the importance of this. They felt that creating a beautiful product was a way to honour the women and girls who received the kits.

Right now, when we do the DfG Kit, it's good quality - we wash first, iron...even the ladies, we ask them to clean their hands, Pipi Sarah wants to give it to the people already good. So if we look at the quality it's good already. 
Her statement reflects the intentions of MKO and NKP who shared with me their belief in the importance of sewing a high-quality kit (see Chapter 6).

What Sarah valued most about the programme was the relationships built with the women who sew the pads and the way she could see their lives being changed by the DfG employment. She said:

I think what I most value is the women that I work with that are making them. Yeah, the relationships we've built going through the process. And because it's combined with another project and the training. I value the fact that I see changed lives in the women who are making them.

Sarah talked extensively about these women, taking the time to tell me each of their stories and how they benefited from and contributed to the programme. She would start by telling me where they came from and then describe the turning point in their lives that came with joining the sewing team. Then she would speak in detail about their roles and the contributions they now make to the programme through their work. For example, one of the first women hired by the programme was a young mother who lived with her son in "an empty lot with a shack with a tarp over it". The woman was allowed to take her baby to work and eventually earnt the money to find more stable housing. Sarah described how her agency grew along her journey - finding a home, a community and the ability to forge her own career path:

From there she was able to get a small little one room thing and from there she was able to get an apartment. And so it was watching the fact that she was able to come out of where she had been into a better situation. And she had a job where her son could come every day and be part of the community there. And she eventually moved on to another job.

While this woman's story was one of agency, Sarah shared a journey of confidence in NSL's story, starting from when they first met her as a health patient who had travelled from her village in Burma:

NSL was this quiet girl. She was just so - it just looked like somebody had beaten her down or something. And so we had a meeting and I said, "Okay, would you be interested in training here and learning how to train others?" And she said yes she would. And in a year we watched her grow from this quiet [girl, to] cooking for everybody, and training [staff], and it was just watching her grow.

In working for the DfG programme during her recovery, NSL was offered training and employment so she could learn skills and earn a living. Over time, these effects and the spirit which she put into her work made NSL grow in self-confidence, which reflected in the way she could then give back to the programme. It is well documented that meaningful employment helps new members integrate well and 
feel comfort in a host society (de Neve, 2018; Searle, 2012; Ager \& Strang, 2008), and NSL is a clear example.

Sarah held respect for the diversity of the team and the individual journeys of the women. Through her eyes, the women on the sewing team are one of the greatest parts of the DfG programme. And the programme's ability to bring confidence and agency to their lives in transformative ways is its greatest strength.

These various answers do not point to one major strength of the Partners DfG programme, but to the programme being meaningful to different members in different ways. There didn't seem to be a rehearsed or rhetorical message coming from staff but mostly personal impressions. Reed (2007) and Michael (2005) express that Appreciative Inquiry can be beneficial in studies of work programmes because participants are more willing to speak freely about their positive experiences than about weaknesses or downfalls. The participants talked about strengths that related to both the women who will receive the kits and to themselves and what they get out of the work. Some spoke at length in answer to these questions and seemed very happy to focus on the positive experiences of being involved in the programme.

\subsection{Challenges}

On the other hand, Appreciative Inquiry doesn't exclude discussion of difficulties (McNamee, 2003). Rather, recognition of barriers can add to a research project's appreciation for a team because we can see how they work despite challenges. Following this methodology, I also asked participants what they perceived the challenges of the programme or working in the programme to be. The following responses were a mixture of replies to this question, and comments that arose through the appreciative process - a development that is predicted by Reed (2007).

\subsubsection{Challenges for the programme}

Two staff members talked very seriously about kit material resourcing as the greatest challenge to the Partners DfG programme. The Migrant Programme Coordinator expressed worry that the US was the only place from which the flannel for the front of the liners could be sourced. She said this process was difficult and could become unreliable, "if we run out of flannel it is the biggest challenge. We cannot keep making any kits". Sarah also discussed this problem, at length.

I cannot find flannel. And the PUL lining, the plastic lining that goes in between [the layers of cotton in the shield], I haven't found that over here yet either... I haven't had a reply from DfG as to where we can get it, [...] even though I've been asking for a long time. And it used 
to be colder in the winter here, and so in the past there was flannel in the markets. But now it's not cold enough to have flannel.

Discussions of possible future expansion of the programme seemed to be shadowed with this pressing concern. For the leaders of the programme, there was real worry that the impact on women could be compromised by problems of logistics.

Other comments about the programmes' challenges focused on reasons why those who receive DfG Kits may not use them. Eh Moo and Partners' Education Coordinator discussed the potential inconvenience of washing and drying the kits that echoed the reasons many Shan students don't use them (Bardsley, 2019).

I think, some of the students, some of them, if they have money to buy the pads, I think they don't want to use the DfG. (Eh Moo)

For the girls who are near the city, these girls I think they don't use them [laughs]. Because when you use it, you need to clean it and wash it, so sometimes, they don't want to... but for the rural area, I think very helpful for them. (Education Coordinator)

Likewise, NSL said that for women living in Mae Sot and travelling home often to see family, the DfG Kits can become cumbersome:

For Kotex, it's comfortable when you're travelling, you don't need to wash it. For DfG, it's good for night time and when I'm staying at home, but not for travel... For people who are lazy, it's not good to use in the day time. For Kotex, you can just throw it so it's easy.

Overall, these quotes from staff and sewists point to an important consideration for the kit: it may increase women's workloads. There is a line of academic criticism of some 'development' projects that target women as they have the potential to add to women's 'triple-burden' responsibilities and workloads in settings where they already bear the brunt of household and day-to-day work (Ruxton \& Oxfam, 2004; Wanner \& Wadham, 2015). Because the DfG Kits have to be washed and dried, it is possible that women would spend more time and energy using them to manage their periods than disposable pads. On the other hand, the women in this study cited lack of disposal facilities (in Mae La) and the need to go to the store to buy disposables as reasons that disposables may increase exertion. In the end, the choice of $94.1 \%$ of the students and both migrant women surveyed to use the kits shows that they assessed the cost of washing and drying the DfG Kits as lower than its benefits. 


\subsubsection{Barriers for DfG users}

The Mae La students themselves also shared with me some difficulties they have with the DfG Kit. One student described a resource limitation, referring to the small bar of soap provided in the kit to help wash the pads, and the problem of it running out: "The soap is not enough for us [...] If we use with soap, it is easy to wash them and not smell bad, but if we use washing powder, it smells bad and doesn't clean easy" (Mission student). This student said that the kit could be more useful if it came with a larger bar of soap. The other main challenge mentioned by the students was that sometimes, especially during the rainy season, there wasn't enough time to dry the liners between uses and it was possible to run out. One student said that it takes two to three days to dry the fabric when the air is particularly humid.

Sometimes when it rains, it doesn't dry easily, so we change to using pads. Cos it's wet, it doesn't dry easily. So during the winter if it doesn't rain, all the time we use DfG Kit. (IRC student)

If we go out - to school, to church, to play with friends, but if we are home we prefer to use nothing. Sometimes we worry about running out of the flannel. (IRC student)

This comment from the IRC student stands against some of the positive effects discussed previously. While some students said they enjoyed not using pads when they were home, students that wanted to wear the pads all the time may not have had this option in the rainy season. Furthermore, if students were no longer buying disposable pads because of their kits, they may have felt they had to stay at home more often because of the restriction of drying the pads in the kit.

This issue is discussed in other evaluations of reusable menstrual pad programmes. Just as in dry climates there can be insufficient water to wash the pads, in wet climates or seasons. there is an extended time needed to dry the materials (VanLeeuwen \& Torondel, 2018; Wilson et al., 2012). What's more, students said that they usually dry the kits underneath the house in the wet season to avoid them being wet by rain. According to Days for Girls training as well as other MHM researchers, this is not an ideal practice because sun-drying can naturally disinfect the kits (Days for Girls International, 2016; Kaur et al., 2017). So while the kits are largely praised by the students and women who receive them, they also admit to challenges in using them. The DfG Kit design has been deeply researched and trialled by Days for Girls International, but just as in any development programme, the design should be an ongoing process informed by the users of the kits and issues that they raise such as these.

This research has shown that in development programmes, this process needs to be place-based and specific. It should be informed by those with 'embedded knowledge' (the recipients and those that live 
in the context) and not based on general prescription (Haraway, 1991; Sommer, 2012). The placespecific knowledge shared by participants about the difficulties of obtaining soap in a refugee camp and the needs of increased flannel in wet climates was shared with other staff.

\subsection{Further analysis and considerations for the future}

This section takes a deeper look at the structures within the DfG programme and the structures it sits within. I provide a feminist analysis of knowledge, power, cultural and gendered aspects to the programme. I evaluate some more strengths of the programme according to MHM literature and bring up some critiques that may be useful to consider as it grows and develops.

\subsubsection{How does Partners learn from the communities it serves?}

In this research, I saw some of the ways that Partners learn from the communities in Thailand where they operate the DfG programme. Partners is at the start of their formal research journey, with their first large-scale survey being developed as I worked with them in Chiang Mai. But they are in close relationship with most of the communities they serve and so most of their learning from feedback has been a more informal, natural process.

As well as during DfG programmes, the women in the migrant community connected with the Migrant Programme Coordinator through the church where she worked and they gave their feedback there. This relationship-based programme monitoring is in line with social practice theory, where evaluation is an everyday routinized habit which helps to evolve practice (Zozimo, 2019). The Migrant Programme Coordinator, a staff member from Burma, was able to personally connect with the migrant women she works with. She either responded to needs herself or relayed community needs and feedback to the DfG team with whom she was in constant communication. She talked about two types of feedback she received from the migrant community:

I have heard feedback from the community that it's really helpful. Some of the ladies will come and say "thank you, [the kit] is really useful". And they will come to the church. And before, they will wear a lot of sarongs and stay home and not go out. And right now they will come to church and go out a lot. Not only one person's story - a lot of people have come to tell me that.

Every time I have questions, I can ask, "Is it okay? If you need anything just let me know". And I don't have any questions. From my experience, every time I do the training, the ladies and girls don't ask about the DfG Kit, they ask, "Why I'm itchy" ... about health. For me, if anybody is itchy, I will go and buy medicine from the pharmacy.

This second statement connects with recent MHM theories (Bobel, 2019; Graf \& Patrick, 2015), where 'menstrual literacy' and 'reading the body' are vital for empowering women in MHM, even more so 
than the menstrual provisions. They say that when time is taken to offer purposeful, context-appropriate, community-supported education about periods and the body, women live longer, feel less menstrual stigma, and have healthier (physically and mentally) relationships with their menstrual cycles. This research project shows that women and girls want to know more about their bodies. They value the menstrual education that they have received, and they want to understand more.

Another way for development programmes to learn from their communities is in cultural competence (Charleston, Gajewska-De Mattos \& Chapman, 2018). As part of my interview with Sarah, I asked the question: "In what ways has the programme changed to work with people's cultural beliefs over time?"

One reflective response was related to the purchase of underwear to go inside the DfG Kits. Sarah discussed the challenge of finding appropriate styles for the various communities of women and girls. Through her relationships with communities as well as local staff, she has come up with a set of guidelines for those purchasing the underwear: She said that sparkles, the red and orange colours of the monks' robes, and animal prints are inappropriate for at least some Karen women. Sarah said that growing in understanding of the communities she works within has "made us more aware that we need to be more sensitive to the fabric". Sarah's response shows humility and commitment to responding to learned understandings which is in line with feminist understandings of development - that those in power should strive to understand and act on the knowledge of the women with whom they work, especially when working cross-culturally (de Lima Costa, 2016).

\subsubsection{Knowledge and power}

An area where Partners found it harder to prioritise women's knowledge was in response to the 'hair washing' question (mentioned in Ch 6). Students in Mae La said they had been told or experienced themselves that washing hair during menstruation can be bad for one's health or fatal and they asked me if this was true. As I had been asked this a number of times during my interviews, I decided to ask Partners staff how they had or would respond to this question themselves during a DfG training.

One staff member said they would try to understand more about their knowledge, "I would ask them the question back - why do you do this?". One said they would try to explain their perspective on the question as a personal answer, "You can say, "For me, I'm happy to wash my hair because..."” (to be honest, this is also what I answered when the students asked me the question). And one said that their answer would depend on if they understood there to be danger or harm involved, "If it's not going to do any harm then it doesn't really need to be challenged." He also said that if this is the case, the challenging of practice would usually be done by local staff: "It doesn't have to be coming from us as foreigners. Usually it can be better if it comes from local staff." The discussion seemed thoughtful and nuanced. The staff were obviously aware about the need to treat the people they work alongside with respect and that "you don't want to be belittling their view." But at other times during my research, I sensed a wider power/knowledge gap. 
In one of my discussions with one staff member, there was more criticism in her tone around the mentioned menstrual practices, "There's so much fear around whatever they've been taught."

“...And they don't wash, they don't bathe and they eat strange food. I say strange food...

It's because their parents have passed it down to them."

The use of the word 'strange' and the links to fear are reminiscent of modernisation rationale where non-western practices and belief systems are 'other' as compared to Western science and knowledge (Catungal, 2011). This way of thinking has implications on cross-cultural relationships. If there is already a power imbalance between people of different cultural backgrounds, this gap can be widened.

Such a gap was apparent in the way that the sewing women talked about the Western staff. I asked them questions about their knowledge of the programme and how they thought it could develop in the future. The participants displayed a hesitancy when I asked about future changes in a way that made me think they were displaying 'grangai' - not wanting to put Partners down by suggesting improvements. While some did answer with their own ideas, other answers referred back to Sarah, for example: "It depends on Pipi...If Pipi wants another thing [then we can change it]..." Sarah was positioned as the authority of knowledge. While this came out of the women's respect for her and the wisdom she has built within the programme over many years, it also seemed that they were forgoing the opportunity to share their own knowledge.

Given Sarah was the sewing women's boss, it is likely that these responses were largely impacted by workplace hierarchy which can be overt in Asian countries (Kwan, Tuckey \& Dollard, 2014). But there were other moments where this knowledge hierarchy was evident. One of the Karen staff members discussed with me the impacts of the programme through the lens of knowledge in the following way:

Partners gives knowledge about the women parts and so how they menstruation. [...] Because we give knowledge so that they will know how to be careful with their kits and then give knowledge like cleanliness and also, in the book they tell about if they want to have a baby with the months. That is a kind of, we educate them like that so that they will know.

In this staff member's three-times mentioning of 'giving knowledge' in the same response, I can see a clear aim of the programme which is also repeated across Days for Girls public discourse (Days for Girls International, 2019a) - that there is a set of knowledge, particularly coming from Western medical practice which is beneficial to indigenous and other ethnic groups who do not yet possess it.

I do not comment on these aspects to merely apply criticism. In fact, the idea of passing on knowledge to increase menstrual literacy is one of the key strengths this thesis recognises. But by exploring the attitudes to menstrual knowledge in this research, I want to acknowledge the complicated relationship within development MHM whereby knowledge hierarchies position Western powers at the top. This is 
not to say that the work done within these systems is not helpful but that an awareness of the power and knowledge inequalities in this work is always needed (Jenkins et al., 2019; Jones et al., 2012).

\subsubsection{Men's involvement}

In this last section of analysis, I touch upon one of the comments made by a local staff member about the MHM programme. In my research, I wanted to understand how the programme went beyond women in its scope. Feminist epistemology's gendered approach is not limited to women so I asked staff the question, "What is it like talking to the men in Partners about your work in menstrual health?". The staff said that medical staff would usually be open to the conversation, but it was not the case for everyone. One of the answers that struck me the most was: "For the DfG, if we are talking about DfG, if the guy came, he'd be like, "I don't want to hear about it." He would go away, because this is the girls' stuff. Just like [Partners Senior Staff Member]."

According to recent MHM research, an important way to reduce the stigma of menstruation is for men to be involved in conversations about it. Their involvement helps the participants and those hearing the conversations to see menstruation as a normal part of life and not something that must be concealed (Bobel, 2019). As mentioned in the previous chapter, the Days for Girls Programme, by nature of being an MHM programme, sits within a larger MHM world system which can tend to privilege the importance of products that cover up menstruation over directly addressing the structures of stigma and shame we associate with women's 'leaky bodies' (Longhurst, 1991). Again, I stress that menstrual products are practical and not the cause of menstrual stigma, but when we look beyond the DfG Kits we can see that open discussions and education about menstruation, that involves men and wider communities, is a more profound way to decrease stigma.

What's more, it has been shown that when men are educated on menstruation, there is more scope for women and men to jointly and successfully make community and policy decisions that allow for ease of menstrual management (Mahon el al., 2015). These decisions can involve ensuring adequate facilities, provision of products and wider education to increase menstrual literacy and decrease menstrual stigma.

Including menstrual education for boys could be meaningful for the female students I interviewed in Mae La. They voiced the challenge of washing menstrual materials out of sight of the boys in their boarding home and their discomfort of discussing menstrual matters with males: "We worry that people are gonna come and see when we wash the kit. [...] We just worry about other people or boys seeing." (IRC student). 
One way that Partners aims to overcome menstrual shame in front of men is by beginning Days For Girls International's new 'Men Who Know' programme. According to Days for Girls International (2019): "The goal of Men Who Know is to instruct accurately about menstruation and reproductive health, to help open conversations about real strength vs violence, and to create opportunities for men to stand together in greater support of united communities." (Para 4).

In our interview, Sarah expressed a desire to bring this programme to the communities with whom Partners works in the DfG programme. This plan would help to create what Bobel (2019) describes as a "360-degree approach" where girls are seen as the expert on their own menstruation but are also supported by the structures they live within.

Everyone in her sphere of existence must substantively engage the often-uncomfortable topic of menstruation and take responsibility for creating a world where menstruation is neither a cause for secrecy, nor shame, nor a tempting opportunity to provide a too-simple consumerist solution for a very complicated social problem. (p. 298).

If Partners were to embrace this '360-degree' attitude, it would require analysis of the current state of 'men who know' within members and stakeholders. In my discussion with Partners staff, I asked how they thought a more gender-inclusive programme would be received in the communities where they work. They said that in some places, for example Bangladesh and their programmes in the Middle East, there would need to be further thinking about cultural implications of such a course. But they said they were encouraged by the training they had already undertaken with Community and Village Health Workers where the male students were "very open and wanted to learn about it." (Southeast Asia Operations Manager, Partners discussion).

Many male Partners staff members have welcomed and engaged with conversations about periods in the MHM programme, and this engagement is increasing. However other staff's avoidance could be inhibiting a spirit of a 360-degree MHM response. The Partners DfG programme and many of the communities where they work seem ready for a development where men and women are empowered through and for MHM. But it might take a more whole-of-Partners response to enable a whole-ofcommunity response as discussed.

In the world of menstrual health, we can see a late and gradual shift to emphasising the importance of men's involvement (Days for Girls, 2019; Mahon et al., 2015). Those that are on that journey (and those that are not!) could learn from aspects of Partners programme such as teaching MHM content to both male and female community health workers and having male staff that talk about and invest interest in the programme. And they could learn alongside Partners about what it might look like to 
involve more senior leadership in this shift, and embracing programmes that target men such as "Men Who Know".

\subsection{Conclusion}

This chapter has delved further into the strengths of, and the barriers to, the MHM programme and explored its knowledge-based, power-laden and gendered aspects. I have shown how kit quality and women's employment contribute to increased agency, confidence and 'changed lives' for the women from Burma who make and receive the DfG Kits. At the same time, this programme faces resourcebased, environmental and practical challenges that limit the ways this confidence and agency can be achieved.

Partners has learnt from the communities it serves in a variety of ways - growing in cultural competence and through their close relationships with participants. Their journey can also inform wider understandings of MHM in development. This research has shown that there is often room to look critically at the structures of power in knowledge that can occur between staff and participants, and that while men's involvement in MHM is generally low, there can be benefits encouraging a whole-ofcommunity response. 


\section{Chapter 9 - Conclusion}

\subsection{Summary of findings}

This mixed-methods research has worked within a feminist epistemology informed by Appreciative Inquiry methods and Follow the Thing to understand how humanitarian MHM care can be effective. It has shown and how those involved in and benefiting from MHM processes might further improve women's safety, confidence and right to thrive. Through analysis of Partners Relief \& Development's MHM programme for migrant and refugee women in Thailand from Burma, I have provided insight into perceptions of MHM, how it can affect confidence and agency, and how this can affect wider understandings of MHM. The main findings of this research project can be summarised according to the first two research questions (the third will be addressed later in the chapter):

1. How can public perceptions of menstruation be affected by discourse and menstrual literacy education?

In the literature review of this thesis, I shared research that showed how perceptions of menstruation are shaped by culture and experiences of displacement but that perceptions and experiences of menstruation are not homogenous, even within cultures. I also noted that menstrual practices and stigmas are shaped by culture, but also by NGO practice and discourse.

For the women I interviewed in this research, perceptions of menstruation were impacted by the Partners MHM programme. They reported that the education provided helped them to learn more about the menstrual cycle and its effects on their bodies - increasing their confidence in their menstruation. Stigma was also reduced through open menstrual literacy conversations, especially for the Mae La students who had felt embarrassed to talk about menstruation.

As suggested by other MHM research (Bobel, 2019; Clatworthy et al., 2018; Sommer, 2012), positive perceptions of menstruation can be further progressed through even more open discourse, more menstrual literacy education and the involvement of wider communities in MHM discussions. This finding is important to both Partners' programme and other MHM programmes worldwide - reducing stigmatised perceptions of menstruation can help with access to menstrual care, can accelerate the addressing of menstrual needs in a community and can help women and girls to feel more confident in themselves and their menstruation.

\section{How does Partners' Menstrual Health Management (MHM) relief affect confidence and agency for} refugee women on the Thai-Burma border?

As discussed in Chapter 5, a key strength of the programme was that it connected the stories and contributions of many women along the 'lifeworld' of the DfG Kit commodity chain. These 
relationships had implications for their confidence and agency. For example, the connections and relatability the students at Mae La felt with their MHM teacher helped them to feel more confident talking about the topic of menstruation. And the connections that the women who sewed the kits felt with those who received them helped them to feel more purpose in their work - that through their jobs they had the agency to help women who shared similar stories of displacement.

A sense of confidence and agency in menstruation was also reported by the students and migrant women as a result of the DfG Kits. In surveys and interviews, women and girls said that the kits helped them to feel more confident, as compared to the disposable pads they had previously used, because they were more comfortable, easier to use, and made them feel safer and healthier. They also reported effects on agency. The migrant women interviewed said the money saved from not buying disposable pads could now be used to contribute to food and financial security for their families. And the students at Mae La reported increased agency in terms of mobility - that they were more confident to participate in community activities during menstruation because of their comfort and confidence in the DfG Kit. Furthermore, the women who sewed the DfG pads felt confidence and agency because their employment gave them financial stability, a sense of purpose and skills for their own futures which they could teach to others.

To further outcomes in confidence and agency, the MHM programme can continue to listen to the needs and dreams of the women it serves and involves. It could facilitate more opportunities for sewing women to bring kits and sewing knowledge to their home communities, as desired by the participants. And it could tailor the kits and distributions in a way that responds to the needs expressed by the students at Mae La - considering feedback on pad size, number of products (or flannel sheets, in the case of DfG Kits) and other provisions such as soap and underwear.

\subsection{Limitations}

Viewing the research project through a reflective lens has revealed ways that this project has been a limited, situated and time-restricted process.

While I addressed many of these in Chapter 4 (Research Approach), including my partiality, limited time in the field and translation, I conclude here with some final reflections. Appreciative Inquiry and Follow the Thing are complex, comprehensive and demanding processes that deserve deep attention from the researchers who claim to use them (Christophers, 2011; Reed, 2007). While I have used aspects of these approaches, and especially tried to keep to the principles of Appreciative Inquiry, this research project does not claim to have exhaustively applied them.

Another limitation is the evaluative 'case study' approach. While the findings were not meant to be generalisable, this research was limited to participants in Thailand who were involved with one MHM 
programme - that of Partners Relief \& Development. It did not cover the programme's impacts in Burma, Bangladesh or the Middle East. The experiences of women in this research may have differed from those in other locations where Partners' programme operates. Furthermore, while most of the staff and sewists involved were interviewed, and most of the Mae La students involved were interviewed or surveyed, I only spoke to two of the Mae Sot migrant women who had been part of the MHM programme, and so findings were not representative of all migrant women in Mae Sot. I also did not carry out formal interviews with any men in this research project, which may have enhanced or modified its perception of the programme.

Perhaps the greatest limitation is that I am a foreign researcher without lived-experience of Karen and other Thai/Burmese cultures. Cross-cultural research was educational and rewarding but also posed challenges in terms of communication, data collection and analysis. I could not carry out research that was deeply culturally informed on my part, despite my efforts to learn about the people with whom I was researching. I did increase my understanding of the complexities of cultural customs and their sources and the impossibility of simplifying aspects such as 'Karen menstrual practices'. Other research has found that women's indigenous knowledge often tells menstruation differently than we have seen through the colonial and western lens (Baldy, 2017; Murphy, 2011; Wall et al., 2018). I wanted to learn of the culturally-informed menstruation knowledge or practices of the women I interviewed, but my distance from the participants was too vast. It seemed that if there were positive 'Karen' constructions of menstruation, they were buried to me under complexities of cultures and language barriers.

\subsection{Contributions to development MHM}

While I have already started to discuss key lessons in answer to Questions 1 and 2, further overall contributions of this thesis can be summarised in answer to my third research question:

3. How can lessons learned from this case study inform wider understandings of MHM in development?

While there is an increasing number of studies into menstrual health in Development, this is the first study in the context of refugees and migrants in Thailand from Burma. Furthermore, it is the first intentionally strengths-based academic evaluation of a similar programme. The strengths of the programme, its challenges and the words and drawings of those involved highlight the need for a tailored and situated development MHM response: one that responds to the knowledge of and adequately supports those who experience menstruation in precarious living situations.

One of the key lessons from this research is the importance of culture-specific and place-specific considerations in in development programmes, both in the design process and in their ongoing evaluation. The participants of Partners' MHM programme shared many insights into how the DfG Kits 
could be improved to be more relevant to their context and how the menstrual health education could be extended. They also alluded to ways that they could see the programme developing in the future, for example through sewing education and providing the DfG in new communities. These women have embedded knowledge that will be invaluable if Partners wants to increase the confidence and agency impacts of its programme. The women's contributions show that development programmes should be informed by those with 'embedded knowledge' (the recipients and those that live in the context) and not based on general prescription.

Despite its limitations, the 'situated' case study nature of this research has also been a strength. This deep look at the practice of one NGO has allowed critical evaluation of how individuals and relationships can affect and be affected by an MHM programme, in a way that gives women and girls confidence and agency. My collaboration with Partners Relief \& Development and the women involved allowed rich, contextual insights into MHM processes, effects, education and stigma. The personal approach allowed me to explore the real-life, nuanced ways that women can interact with menstrual health and with NGO activity. I was able to bring the expressions of the women I interviewed alongside academic literature, compare MHM programmes, criticisms and practice recommendations to generate analysis and conclusions.

I came into this research project with an idea that I would be evaluating how menstrual pad provision reduces stigma around menstruation. But along the way I learnt that MHM stigma is much more complex than simply menstrual provision. Through MHM literature and my own research, I began to see that perceptions around menstruation are shaped not only by communities but by the programmes and campaigns that aim to alleviate period poverty and stigma themselves. While their products can give women feelings of agency and confidence during menstruation (and Partners' pads also importantly provide employment), discourse around menstrual provision has also been critiqued for furthering the 'culture of concealment' (Bobel, 2019; Houppert, 1999). The suggestion for development MHM to best reduce stigma is to encourage open, inclusive, educational discussions around menstrual literacy alongside providing menstrual products according to the expressed needs of communities (Bobel, 2019; Graf \& Patrick, J. H., 2015; Mahon et al., 2015; Sommer, 2012; Sommer, Schmitt, \& Clatworthy, 2017). In particular, this research has shown that education programmes should be: responsive, incorporating questions from participants into future trainings, and following up with training where knowledge may have been lost; and inclusive, involving men and boys and recognising their role in reducing menstrual stigma, which can contribute to women's confidence and agency.

Finally, this research reflects on and challenges some of the discourse in the 2015-2030 Sustainable Development Goals. I return here to SDG \#5, 'Gender Equality': “[...] Providing women and girls with equal access to education, health care, decent work, and representation in political and economic 
decision-making processes will fuel sustainable economies and benefit societies and humanity at large" (United Nations, 2015, para. 3).

The possible increased access to education and work and the 'health' care provided by the Partners' MHM programme will have implications for economies and societies. But just as importantly, programmes to support women can support them for their own sake. They can encourage women's agency to participate in activities and earn a living to benefit their communities - and they can inspire women to feel more confident in themselves. Most of all, these things can be best achieved when women's situated contribution and knowledge is valued at the centre of a programme.

\subsection{Where to from here?}

A number of recommendations can be drawn from this study. But as a principle of Appreciative Inquiry, these recommendations should most importantly come from participants in the programme at hand (Reed, 2007). Therefore, these recommendations summarise the 'Dreaming' expressed by staff, DfG pad sewists, Mae La students and migrant women who have been a part of Partners' MHM programme. I have added ideas from my observations of the Partners MHM programme, in consideration of MHM literature. Some of these recommendations serve as a start to the 'Designing' process in Appreciative Inquiry - informing the ongoing changes that Partners is already making to the programme. Others are discoveries made through this research, where the practice of the programme and the women involved are already increasing women's agency and confidence, and could be applied to other MHM programmes in development and humanitarian settings.

Recommendations:

- DfG Kits are made and distributed in a way that is relevant to the context of the recipients. Feedback from recipients is sought and considered concerning size of pads, number of products (or flannel sheets, in the case of DfG Kits) and other provisions such as the amount of soap and types of underwear.

- MHM providers work with local women to address menstrual health issues, listening to their situated and embedded knowledge of cultural issues, environmental factors and practical needs. Developments and expansion in MHM programmes are led by local women, such as the women who sew the DfG pads at Partners who would like to do more sewing training and DfG distributions with women in their village.

- Where possible, production of MHM products should be done in ways that empower women, such as Partners' sewing programme that employs migrant women, teaches them skills they can share, and connects them to other women and girls that receive the kits. 
- Beyond the provision of menstrual products, MHM provision includes strong menstrual literacy and teaching on how to 'read the body', as this has implications for lowering stigma and increasing menstrual health.

- The topics of menstrual pain, heavy bleeding, recommended food and hygiene methods are discussed during MHM training - in open and sensitive ways.

- Menstrual literacy and other health education is repeated or 'refresher courses' are offered, to further ongoing relationships and to help participants to retain information.

- MHM campaigns and discourse (both to donors and participants) discourage the idea that menstruation is a dirty process that must be concealed, but a natural process that women can have power over through menstrual literacy and the ability to choose menstrual products that work for them.

- MHM providers are reflexive and recognise the ways that they have power over the women they work with - whether in knowledge, position in an organisation or through representation. Efforts should be made to empower women in each of these areas.

- MHM education and conversations are expanded to other genders - particularly to men who have influence over menstrual stigma and community decisions that affect menstruating women. MHM providers actively engage men in MHM initiatives, both within their own staff and in the communities where they work.

\subsubsection{Areas for further research}

While I was limited as a foreign researcher, further research by someone with embedded knowledge could include a comparison between different cultural groups from Burma - of experiences of menstruation, and experiences of NGO menstrual health programmes. This could include further examination of the roles and perceptions of menstruation in specific cultural contexts.

This research has gone some way in understanding how the refugee and migrant journey affects women's experiences of and perceptions of menstruation. But there is further research to be done particularly addressing how aspects of displacement and trauma affect menstrual experiences and how menstruation is experienced at other stages of the displacement journey, before the host country is reached.

Through the aspect of visual methods used in this research, I see potential for visual approaches to tell personal stories and to extend understanding of participants' voices more effectively than in traditional research methods. For example, a study that more thoroughly uses visual methods (mapping, photovoice or film-making) could lend further insight into how menstruation really affects mobility within the spaces of daily life. 
On another note, I have suggested that a whole-of-community response to menstruation is an important way to reduce stigmatised discourse around MHM and respond to menstrual needs. To support MHM programmes who wish to do this, research could explore the influence of menstrual health programmes on families and communities, rather than focusing solely on women's experiences. It would also be interesting to see more data on the impacts of a whole-of-community menstrual response that goes beyond the conceptual.

Finally, there is potential for further research into the conceptual and theoretical aspects of menstrual stigma. This includes study on the 'culture of concealment' and how MHM organisations work with concepts such as 'dignity' and 'shame'. Further study could explore how discourse in MHM positively and negatively affects public perceptions of menstruation, and women's perceptions of their own bodies. 


\section{References}

Adams, K. M., \& Robinson, D. W. (2001). Shame reduction, affect regulation, and sexual boundary development: Essential building blocks of sexual addiction treatment. Sexual Addiction \& Compulsivity, 8(1), 23-44.

Aflaq, F., \& Jami, H. (2012). Experiences and attitudes related to menstruation among female students. Pakistan Journal of Psychological Research, 27(2), 201-224.

Ager, A., \& Strang, A. (2008). Understanding integration: A conceptual framework. Journal of Refugee Studies, 21(2), 166-191.

Alfath, A., Firman, F., \& Syahnair, S. (2018). The effectiveness of information service using contextual teaching and learning in preventing sexual abuse. International Journal of Research in Counselling and Education, 2(2), 58-63.

Alldred, P. (1998). Ethnography and discourse analysis: Dilemmas in representing the voices of children. In J. R. Ribben \& R. E. Edwards (Eds.), Feminist Dilemmas in Qualitative Research: Public Knowledge and Private Lives. London, UK: SAGE Publications.

Amnesty International. (1999, June 1). The Kayin (Karen) State - Militarization and Human Rights. Refworld. Retrieved from https://www.refworld.org/

Baldy, C. R. (2017). Mini-k'iwh'e:n (for that purpose - I consider things): (Re)writing and (re) righting indigenous menstrual practices to intervene on contemporary menstrual discourse and the politics of taboo. Cultural Studies - Critical Methodologies, 17(1), 21-29.

Barbara, D. B., \& Benjamin, C. (2006). making sense of qualitative research: The qualitative research interview. Medical Education, 40, 314-321.

Bardsley, J. (2018, August 27). For Girls. Retrieved from https://blog.partners.ngo/

Barnett, M., \& Weiss, T. G., (2008). Humanitarianism: A brief history of the present. In M. Barnett \& T. G. Weiss (Eds.), Humanitarianism in Question: Power, Politics, Ethics (pp.1-48). Ithaca, NY: Cornell University Press.

Barron, I., Mitchell, D., \& Yule, W. (2017). Pilot study of a group-based psychosocial trauma recovery program in secure accommodation in Scotland. Journal of Family Violence, 32(6), 595-606.

Bartholomew, K., \& Calder, R. (2018). Burma Landscaping Report: Spring (October, 2018). Retrieved from http://www.springaccelerator.org/

Beitz, C. (2013). Human dignity in the theory of human rights: Nothing but a phrase? Philosophy \& Public Affairs, 41(3), 259-290. 
Benner, M., Townsend, J., Kaloi W, et al. (2010). Reproductive health and quality of life of young Burmese refugees in Thailand. Conflict Health, 4(5), 5-14.

Black, C. J. (2003). Translating principles into practice: implementing the feminist and strengths perspectives in work with battered women. Affilia-Journal Of Women And Social Work, 18(3), 332-349.

Bobel, C. (2019). Developing Girls and Menstrual Health in the Global South. Cham, Switzerland: Springer Nature Switzerland AG. https://doi.org/10.1007/978-3-319-89414-0

Brown, C. (2015). Globalization, International Education Policy and Local Policy Formation Voices from the Developing World. DOI: 10.1007/978-94-007-4165-2

Browne, B. C (2013). Recording the personal: The benefits in maintaining research diaries for documenting the emotional and practical challenges of fieldwork in unfamiliar settings. International Journal of Qualitative Methods, 12(1), 420-435.

Budhathoki, S. S., Bhattachan, M., Castro-Sanchez, E., Sagtani, R. A., Rayamajhi, R. B., Rai, Pramila., \& Sharma, G. (2018). Menstrual hygiene management among women and adolescent girls in the aftermath of the earthquake in Nepal. BMC Women's Health, 18(1), 1-33.

Burgess, R. G. (1991). In the field: An Introduction to Field Research. London, England: Routledge.

Burma Link. (2015). Refugee Camps. Retrieved from https://www.burmalink.org/

Butler, A. (2005). A strengths approach to building futures: UK students and refugees together. Community Development Journal, 40(2), 47-157.

Campbell, R., \& Wasco, S. M. (2000). Feminist approaches to social science: Epistemological and methodological tenets. American Journal of Community Psychology, 28(6), 773-791.

Castree, N., Kitchin, R., \& Rogers, A. (2013). Feminist geography. In Dictionary of Human Geography. Oxford University Press. Published online ISBN: 9780191758065.

Catungal J. (2011) Circulating Western notions. In: Wane N., Kempf A., Simmons M. (eds) The Politics of Cultural Knowledge (23-36), Rotterdam, The Netherlands: SensePublishers.

Chambers, R. (1994). Paradigm Shifts and the Practice of Participatory Research and Development. Brighton, England: Institute of Development Studies.

. (1997). Whose Reality Counts? Putting the First Last. London: Intermediate Technology Publications. 
Chandran, R. (2019, January 14). Too scared to return home, Burma refugees in Thai camps face an uncertain future. Thomson Reuters Foundation News. Retrieved from http://news.trust.org

Chant, S., \& Gutmann, M. C. (2002). 'Men-streaming' gender? Questions for gender and development policy in the twenty-first century. Progress in Development Studies, 2(4), 269-282.

Charleston, B., Gajewska-De Mattos, H., \& Chapman, M. (2018). Cross-cultural competence in the context of NGOs: Bridging the bap between 'knowing' and 'doing'. The International Journal of Human Resource Management, 29(21), 3068-3092.

Chatter, J. I. (2013). Dispositions and destinations: Refugee agency and "mobility capital" in the bengal diaspora, 1947-2007. Comparative Studies in Society and History, 55(2), 273-304.

Christophers, B. (2011). Follow the Thing: Money. Environment and Planning D: Society and Space, 29(6), 1068-1084.

Clark, T. (2009). Doing Qualitative Research with People and Organisations: How do Researchers Understand and Negotiate their Research Relationships? (Doctoral dissertation, University of Sheffield, United Kingdom). Retrieved from http://etheses.whiterose.ac.uk

Clatworthy, D., Sommer, M., Schitt, M. L., Ogello, T., Mathenge, P., Mark, M., Khandakji, S., \& Ratnauake, R. (2018). Pilot testing and evaluation of a toolkit for menstrual hygiene management in emergencies in three refugee camps in Northwest Tanzania. Journal of International Humanitarian Action, 3(6), 1-14.

Collin-Vézina, D. (2013). Students affected by sexual abuse. In E. Rossen \& R. Hull (Eds), Supporting and Educating Traumatized Students: A Guide for School-Based Professionals (pp.187-202). New York: Oxford University Press.

Cook, I. \& Tolia-Kelly, D. (2010). Material geographies. in Dan Hicks \& Mary Beaudry (eds.) Oxford Handbook of Material Culture Studies, Oxford: Oxford University Press, 99-122.

Cook, I. (2004). Follow the thing: Papaya. Antipode, 36(4), 642-664.

(2016). Why Ethical Consumption? With Professor Ian Cook, University of Exeter [video file]. Retrieved from https://www.youtube.com/watch?v=-CY686xLKHQ

Cook, I., \& Harrison, M. (2007). Follow the Thing: "West Indian Hot Pepper Sauce". Space and Culture, 10(1), 40-63.

Cooperrider, D. L. (2009). Appreciative Inquiry: A Positive Revolution in Change. San Fransisco, CA: Berrett-Koehler. 
. (2013). Organizational generativity the appreciative inquiry summit and a scholarship of transformation. Bingley, UK: Emerald.

Cooperrider, D. L., \& Whitney, D. (2001). A positive revolution in change. In D. L. Cooperrider, P. Sorenson, D. Whitney, \& T. Yeager (Eds.), Appreciative Inquiry: An Emerging Direction for Organization Development (pp. 9-29). Champaign, IL: Stipes.

Cope, M. (2010). Coding Qualitative Data. In I. Hay (Ed.), Qualitative Research Methods in Human Geography (3rd ed., pp. 281-294). Oxford, England; New York, NY: Oxford University Press.

Cornwall, A. (2004). Spaces for transformation? Reflections on issues of power and difference in participation in development. In S, Hickey and G. Mohan (Eds.), Participation: From Tyranny to Transformation, (pp. 75-91). London: Zed Books.

Creswell, J. W. (2014). Research Design: qualitative, quantitative, and mixed method approaches ( $4^{\text {th }}$ Ed). London, UK: Sage Publications Ltd.

Culling, V. M. (2001). Writing/Righting Menstruation: a Feminist Analysis of New Zealand Women's Knowledge of the Menstrual Cycle (Doctoral dissertation, Victoria University, Wellington, New Zealand). Retrieved from https://viewer.waireto.victoria.ac.nz/

Cupples, J., \& Kindon, S. (2003). Far from being 'home alone': the dynamics of accompanied fieldwork. Singapore Journal of Tropical Geography, 24(2), 211-228.

Dawkins, F. (2018). South Africa implements free sanitary pads for schoolgirls project. Face2Face Africa, March 26. https://face2faceafrica.com/article/south-africa-implements-free-sanitarypads-for-schoolgirls-project

Days for Girls International. (2016). Days for Girls Ambassador of Women's Health Training, Version 51316. Retrieved from www.DaysforGirls.org

_. (2017). 2017 Annual Report (Online Report No. 4). Retrieved from https://www.daysforgirls.org/ (2018). Enterprise Evaluation Report (January 2018). Retrieved from https://www.daysforgirls.org/

. (2019a). Turning Periods into Pathways. Retrieved from https://www.daysforgirls.org/singlepost/2018/06/11/Turning-Periods-Into-Pathways

_. (2019b). Days for Girls. Retrieved from https://www.daysforgirls.org/

. (2019c). Monitoring and Evaluation. Retrieved from https://www.daysforgirls.org/monitoring-evaluation

. (2019d). History. Retrieved from https://www.daysforgirls.org/history 
. (2019e). The Days for Girls Kit. Retrieved from https://www.daysforgirls.org/dfg-kits

De Lima Costa, C. (2016). Gender and Equivocation: Notes on Decolonial Feminist Translations. In: Harcourt W. (ed), The Palgrave Handbook of Gender and Development (48-61). London, UK: Palgrave Macmillan.

de Neve, J.-E. (2018). Work and well-being: a global perspective. In G. H. Council (Ed.), Global Happiness Policy Report (75-128). Retrieved from https://www.worldgovernmentsummit.org/

Dignity. (2019). Dignity: Creating a movement for free sanitary items for all women in New Zealand. Retrieved from https://www.dignitynz.com/

Dolan, C. S., Montgomery. P., Ryus, C. R., Dopson, S., \& Scott. L. M. (2012) Sanitary Pad Interventions for Girls' Education in Ghana: A Pilot Study. PLoS ONE, 7(10), 1-7.

Dolan, C., Montgomery, P., Hennegan, J., Wu, M., Steinfield, L.. \& Scott, L. (2016) Menstruation and the Cycle of Poverty: A Cluster Quasi-Randomised Control Trial of Sanitary Pad and Puberty Education Provision in Uganda. PLOS ONE, 11(12), 1-26.

Dorahy, M. J., \& Clearwater, K. (2012). Shame and guilt in men exposed to childhood sexual abuse: A qualitative investigation. Journal of Child Sexual Abuse, (21), 155-175.

Doyle, J. (2008). Improving sexual health education for young people with learning disabilities. Paediatric Nursing, 20(4), $26-28$.

Druet, A. (2017, September 8). How did menstruation become taboo? A look at the historical roots and theories behind menstrual stigma. Retrieved from https://helloclue.com/articles/culture/how-didmenstruation-become-taboo

Duncan, L. (2017). It's all about me: My life as a nameless, faceless guinea pig of the Dunedin Study. Retrieved from https://www.stuff.co.nz/

Gaitan, D., Daw Tin Shwe, V., Bajcevic, P. \& Gagnon, A. (2019), "Alcohol use disorders among Burma migrant workers in Thailand", International Journal of Migration, Health and Social Care, 15(4), 306-319.

Gaugele, E. (2020). Entangled histories: Fashion and the politics of migration. In H. Henns \& V. Hofmann (Eds.) Fasion and Materiality: Cultural Practices in Global Contexts (260-276).

George, A. (2017). The shame of period poverty is keeping British girls out of school. Let's break the $\begin{array}{llll}\text { silence. } & \text { The } & \text { Guardian, } & \text { December }\end{array}$ https://www.theguardian.com/commentisfree/2017/dec/19/british-girls-periodpovertymenstruation-sanitary-products. 
Girl Effect. (2008, May 22). The Girl Effect: I Dare You to See I Am the Answer [Video file]. Retrieved from https://www.youtube.com/watch?v=-Vq2mfF8puE

Goonj. (2018). Help poor women get access to sanitary pads. Retrieved from https://www.giveindia.org/ Gorelick, S. (1991). Contradictions of feminist methodology. Gender and Society, 5(4), 459-477.

Graf, A. S., \& Patrick., J. H. (2015). Foundations of life-long sexual health literacy. Health Education 115(1), 56-70.

Grant, M., Lloyd, C., \& Mensch, B. (2013). Menstruation and school absenteeism: Evidence from rural Malawi. Comparitive Education Review, 57(2), 260-284.

Grigri, L. (2018). Refugee \& IDP Camps along the Thailand-Burma Border. Retrieved from https://www.burmalink.org/

Hagye, E. \& Armstrong, M. C. (2010). Mobility. Encyclopedia of Geography. Thousand Oaks, CA: SAGE Publications, Inc.

Haraway, D. (1988). Situated knowledges: The science question in feminism and the privilege of partial perspective. Feminist Studies, 14(3), 575-599.

Hennegan, J., Dolan, C., Steinfield, L., \& Montgomery, P. (2017). A qualitative understanding of the effects of reusable sanitary pads and puberty education: implications for future research and practice. Reproductive Health, 14(78), 1-23.

Hesse-Biber, S. N., \& D. Piatelli (2007). From theory to method and back again. The synergistic praxis of theory and method. In S. N. Hesse-Biber (Ed.), Handbook of Feminist Research: Theory and Praxis (pp. 143-154). Thousand Oaks, CA: Sage.

Hill, A. E. D. (2019). From 'women's business' to 'men's business': Exploring connections between vasectomy acceptance and equitable gender relations in South Tarawa, Kiribati. (Unpublished thesis for Masters of Development Studies, Victoria University of Wellington, New Zealand). Retrieved from https://researcharchive.vuw.ac.nz

Hinkin, T., Holtom, B. C., Klag, M. (2007). Developing mutually beneficial relationships between researchers and organizations. Organizational Dynamics, 36(1), 105-118.

Houppert, K. (1999). The Curse: Confronting the Last Unmentionable Taboo: Menstruation. New York, NY: Farrar, Straus and Giroux.

Howitt, R., \& Stevens, S. (2010). Cross-cultural research: Ethics, methods and relationships. In I. Hay (Ed.), Qualitative Research Methods in Human Geography (3rd ed., pp. 4068). Oxford; New York: Oxford University Press. 
Human Rights Watch. (2007). Sold to be soldiers. The recruitment and use of child soldiers in Burma. (Human Rights Watch report: October 2007). Retrieved from https://www.hrw.org/

Human Rights Watch. (2019). Burma: Events of 2018. Retrieved from https://www.hrw.org/

Humanity \& Inclusion. (n.d.). Mae La refugee camp in Thailand, a difficult place to be a child. Retrieved from https://humanity-inclusion.org.uk/

International Federation of Red Cross and Red Crescent Societies. (2018, August). Periods don't stop in emergencies: Addressing the menstrual hygiene needs of women and girls. IFRC WASH, 5(E), $1-8$.

IOM. (2019). Refugee Resettlement and Movement Management. Retrieved from https://thailand.iom.int/

Jackson, C. (2006). Feminism spoken here: Epistemologies for interdisciplinary development research. Development and Change, 37(3), 525-547.

Jenkins, K., Narayanaswamy, L., \& Sweetman, C. (2019). Introduction: Feminist values in research. Gender \& Development, 27(3), 415-425.

Jnanavira, D. (2006). A Mirror for Women? Reflections of the feminine in Japanese Buddhism. Western Buddhist Review, 4. Retrieved from http://www.westernbuddhistreview.com/

Jones, H., Jones, N., Shaxson, L., \& Walker, D. (2012). Knowledge, policy and power in international development a practical guide. Bristol, UK: Policy Press.

Jones, R. (2018, February 20). A 5 minute theology of periods. Retrieved from https://www.thegoodbook.com

Kågesten, A., Zimmerman, L., Robinson, C., Lee, C., Bawoke, T., Osman, S., \& Schlecht, J. (2017). Transitions into puberty and access to sexual and reproductive health information in two humanitarian settings: a crosssectional survey of very young adolescents from Somalia and Burma. Conflict and Health, 1(24), 1-64.

Karen Organization of Minnesota. (2017). Family \& Relationships. https://www.mnkaren.org

Kaur, R., Kaur, K., \& Kaur, R. (2018). Menstrual Hygiene, Management, and waste disposal: Practices and challenges faced by girls/women of developing countries. Journal of environmental and public health, 2018, 1-9.

Keenan, J. (2019, May). It is better to work for yourself than sit and wait for aid, Reason. Retrieved from https://reason.com/2019/04/21/it-is-better-to-work-for-yours/ 
Kemp, F. (2009). Saving face: Understanding conflict in multicultural organizations. Industrial Engineer, 41(5), 39-43.

Khin, Y. (2002). Assessing Adolescent Reproductive Health in the Karenni Refugee Community (Masters Thesis). Mae Hong Son, Thailand.

Kikano, F., Lizarralde, G. (2018). Settlement policies for Syrian refugees in Lebanon and Jordan: an analysis of the benefits and drawbacks of organized camps. In A. Asgary (Ed.), Resettlement Challenges for Displaced Populations and Refugees, (29-40). Retrieved from https://doi.org/10.1007/978-3-319-92498-4_3

Kindon, S. L. (2012). 'Thinking-Through-Complicity' with Te Iwi o Ngāti Hauiti: Towards a critical use of participatory video for research. (Doctoral dissertation, Massey University, Palmerston North, New Zealand). Retrieved from https://hdl.handle.net/10289/6286

Kinsman, J. (1997). Shame reduction in a group setting: A pilot study. Ann Arbor, MI: UMI Microform.

Kitch, J., Richardson, H., Bigg, S., Hammond, E., Hudson, H., Hunkler, S., Jansson, A., Tiedemann, E., Laurent, M., O'Flynn, J., \& Coker-Bolt, P. (2017). Implementing the Days for Girls international menstrual hygiene and reproductive health education in Haiti. OT Practice, 22(5), 25-26.

Kivunja, C., \& A. B. Kuyini. (2017). Understanding and applying research paradigms in educational contexts. International Journal of Higher Education, 6(5), 26-41.

Kupiecki, M. (2015). A brief account of what the feminist methodology of intersectional analysis reveals about terrorism studies. Security and Defence Quarterly, 6(1), 107-114.

Kwan, S. S., Tuckey, M. R., \& Dollard, M. F. (2014). Dominant culture and bullying; personal accounts of workers in Malaysia. In M. F. Dollard, R. B. Nordin \& M. R. Tuckey (Eds.), Psychosocial Factors at Work in the Asia Pacific (177-200). London, UK: Springer.

Lahiri-Dutt, K. (2014). Medicalising menstruation: A feminist critique of the political economy of Menstrual Hygiene Management in South Asia. Gender, Place \& Culture: A Journal of Feminist Geography, 22(8), 1158-1176.

Lahiri-Dutt, K. (2017). Thinking 'differently' about a feminist critical geography of development. Geographical Research, 55(3), 326-331.

Leurs, K. H. A. (2017). Feminist data analysis. Using digital methods for ethical, reflexive and situated socio-cultural research: Lessons learned from researching young Londoners' digital identities. Feminist Review, 115(1), 130-154. 
Haraway, D. (1991). Simians, Cyborgs and Women: The Reinvention of Nature. New York, NY: Routledge.

Longhurst, R. (2001). Bodies: Exploring Fluid Boundaries. United Kingdom, Taylor \& Francis.

Maclean, D. (2014). Livelihoods and Empowerment: A case study of Burmese refugees in Thailand. Global Education Magazine. Retrieved from http://www.globaleducationmagazine.com

Mahon, T., Anjali, T., \& Singh, N. (2015). Putting the men into menstruation: the role of men and boys in community menstrual hygiene management. Waterlines, 34(1), 7-14.

Marcus, G. E. (1995). Ethnography in/of the world system: the emergence of multi-sited ethnography. Annual Review of Anthropology, (24), 95-177. London, UK: Bloomsbury.

McEwan, C. (2001). Postcolonialism, feminism and development: intersections and dilemmas. Progress in Development Studies, 1(2), 93-111.

McNamee, S. (2003). Appreciative evaluation within a conflicted educational context. New Directions for Evaluation, 100, 23-40.

Mcnamee, S., Preskill, H., \& Coghlan, A. T. (2003). Appreciative evaluation within a conflicted educational context. New Directions for Evaluation, 2003(100), 23-40.

Merkin, R. S. (2018). Saving Face in Business: Managing cross-cultural interactions. NY, New York: Palgrave Macmillan.

Merrifield, J. (1993). Putting scientists in their place: Participatory research in Environmental and Occupational Health. In P. Park, M. Brydon-Miller, B. L. Hall, and T. Jackson (Eds.), Voices of Change: Participatory Research in the United States and Canada (pp. 65-84). Westport, CT: Bergin and Garvey.

Michael, S. (2005). The promise of appreciative inquiry as an interview tool for field research. Development Practice, 15(2), 222-230.

Mohamed, Y., Durrant, K., Huggett, C., Davis, J., Macintyre, A., Menu, S., Wilson, J. N., Ramosaea, M., Sami, M., Barrington, D. J., Mcskimming, D., \& Natoli, L. (2018). A qualitative exploration of menstruation-related restrictive practices in Fiji, Solomon Islands and Papua New Guinea. PLoS ONE, 13(2). 1-19.

Mokhtar, M. M., Rosenthal, D. A., Hocking, J. S., \& Nuraliza, A. (2013). Bridging the gap: Malaysian youths and the pedagogy of school-based sexual health education. Procedia - Social and Behavioral Sciences, (85), 236-245. 
Molyneux, (1985). Mobilization without emancipation? Women's interests, the state, and revolution in Nicaragua. Feminist Studies, 11(2), 227-254.

Moser, C. (1989). Gender planning in the Third World: Meeting practical and strategic gender needs. World Development, 17(11), 1799-1825.

Moss, P. J. (2002). Feminist geography in practice: research and methods. Oxford, UK: Blackwell Publishers.

Moyo, J. (2018, July 23). Too poor for periods, Zimbabwe's girls rely on rags, paper, leaves. Reuters. Retrieved from https://www.reuters.com/

Mucherah, W., \& Thomas, K. (2017). Reducing barriers to primary school education for girls in rural Kenya: reusable pads' intervention. International journal of adolescent medicine and health. (June, 2017). https://doi.org/10.1515/ijamh-2017-0005.

Murnane, R. J. \& Willett, J. B. (2011). Methods matter: improving causal inference in educational and social science research. New York, NY: Oxford University Press.

Neiman, A., Soh, E., \& Sutan, P. (2008). Karen Cultural Profile. Retrieved from https://ethnomed.org

Nicholson, C., \& Barnes, J. (2012). Appreciative Inquiry. In J. Hockley, K. Froggatt \& K. Heimerl (Eds.), Participatory Research in Palliative Care: Actions and Reflections. OUP Oxford. Retrieved from https://10.1093/acprof:oso/9780199644155.003.0006

Nyein Ei Ei Htwe. (2016, September 2). The Menstruation Taboo. Retrieved from https://www.mmtimes.com/

Nyein, N. (2019, May 31). 'Women in Leadership Positions Must Speak for All Women'. The Irrawaddy. Retrieved from https://www.irrawaddy.com

O’Leary, Z. (2017). The Essential Guide to Doing your Research Project. London: Sage Publications Ltd.

OCHA. (2018). Staying the Course: Delivering on the Ambition of the World Humanitarian Summit (OCHA Agenda for Humanity Annual Synthesis Report 2018). Retrieved from https://www.agendaforhumanity.org/

OCHA. (2019). Burma. Retrieved from https://m.reliefweb.int/

Oka, R. C. (2014) Coping with the refugee wait: The role of consumption, normalcy, and dignity in refugee lives at Kakuma Refugee Camp, Kenya. American Anthropologist, 116(1). 23-37.

Omata, N. (2017). Unwelcome participation, undesirable agency? Paradoxes of de-politicisation in a refugee camp. Refugee Survey Quarterly, 36(3). 108-131. 
Pads4Girls. (2019). About Pads4Girls. Retrieved from https://lunapads.com/pages/pads4girls

Palomino-Schalscha, M. A. (2011). Indigeneity, autonomy and new cultural spaces: The decolonisation of practices, being and place through tourism in Alto Bio-Bio, Chile. (Unpublished doctoral dissertation, University of Canterbury, New Zealand). Retrieved from https://www.academia.edu

Parker, A. H., Smith, J. A., Verdemato, T., Cooke, J., Webster, J., \& Carter, R. C. (2014). Menstrual management: a neglected aspect of hygiene interventions. Disaster Prev Manag, 2014(23), 437454.

Partners. (2019a). The Founders. Retrieved from https://www.partners.ngo/about/the-founders (2019b). Partners Relief \& Development Volunteers Handbook. Thailand: Partners Relief \& Development

_. (2019c). We are Partners: 2019 Gift Catalogue. Retrieved from h https://www.partners.ngo/takeaction/gift-catalog-2019

Perczynska, O., \& Priess, D. (2014, October 30). Not just a girls' problem: the economic impact of menstrual shame. The Guardian. Retrieved from https://ww.theguardian.com/

Pollio, D. E., Mcdonald, S. M., \& North, C. S. (1997). Combining a strengths-based approach and feminist theory in group work with persons 'on the streets'. Social Work With Groups, 19(3-4), $5-20$.

Quinn, N. (2014). Mixed-Methods Research in a Workplace Setting: Access, Negotiation and Methodological Flexibility. London, UK: Sage Publications.

Rador, A. (2017). We're having a menstrual liberation: How periods got woke. The Guardian.

Retrieved from https://www.theguardian.com/society/2017/nov/11/periods-menstruationliberation-women-activists-abigail-radnor

Rathgeber, E. M. (1990). WID, WAD, GAD: Trends in research and practice. The Journal of Developing Areas, 24(4), 289-502.

Reed, J. (2007). Appreciative Inquiry: Research for Change. Thousand Oaks, CA: Sage Publications.

Reinharz, S. (1992). Feminist Methods in Social Research. New York, NY: Oxford University Press.

Reissman, C. K. (2005). Narrative Analysis. In C. Horrocks, B. Roberts, K. Milnes, \& N. Kelly (Eds.), Narrative, Memory and Everyday Life (pp. 1-7). Huddersfield, England: University of Huddersfield.

Ritu. (2017) Baseline Report. Retrieved from http://menstrualhygieneday.org 
Rogers, B. (2012). BURMA: A Nation at the Crossroads. London, United Kingdom: The Random House Group Company.

Rose, Gillian. (1997). Situating knowledges: positionality, reflexivities and other tactics. Progress in Human Geography, 21(3), 305-320.

Ross, R. H. (2015). 'It depends on us': The experiences of fifteen young Burmese migrants living in the border town of Mae Sot, Thailand (Unpublished Master of Development Studies thesis, Victoria University of Wellington, New Zealand). Retrieved from http://hdl.handle.net/10063/5217

Ruxton, S. \& Oxfam (Eds.), (2004). Gender equality and men: learning from practice. Oxford: Herdon, VA: Oxfam

Saltsman, A. (2014). Beyond the law: Power, discretion, and bureaucracy in the management of asylum space in Thailand. Journal of Refugee Studies, 27(3), 457-476.

Sanyal, S., \& Ray, S. (2008). Variation in the menstrual characteristics in adolescents of West Bengal. Singapore Medical Journal, 49(7), 542-550.

Saunders, K. (2002). Feminist post-development thought: Rethinking modernity, postcolonialism and representation. London: Zed Books.

Schmitt, M. L., Clatworthy, D., Ratnayake, R., Klaesener-Metzner, N., Roesch, E., Wheeler, E., \& Sommer, M. (2017). Understanding the menstrual hygiene management challenges facing displaced girls and women: findings from qualitative assessments in Burma and Lebanon. Conflict and Health, 11(19), 1-11.

Schoonenboom, J., \& Johnson, R. B. (2017). How to construct a mixed methods research design. Kölner Zeitschrift für Soziologie und Sozialpsychologie, 69(2), 107-131.

Searle, W. (2012). New land, new life: Long-term settlement of refugees in New Zealand. (Main Report). Retrieved from http://www.mbie.govt.nz/

Sew for a Girl Canberra. (2017, June 30). What a week! Sew for a Girl Canberra. Retrieved from http://sewforagirlcanberra.com/page/3/

Shravasti Dhammika, B. (2019). Menstruation. Retrieved from https://www.buddhisma2z.com/content.php?id=536

Smith, M. J. (1999). Burma: Insurgency and the Politics of Ethnicity. New York, NY: St Martin's Press, Inc.

Sommer, M. (2012). Menstrual hygiene management in humanitarian emergencies: Gaps and recommendations. Waterlines 31(1-2), 83-104. 
Sommer, M., Hirsch, J. S., Nathanson, C., \& Parker, R. G. (2015). Comfortably, safely, and without shame: Defining menstrual hygiene management as a public health issue. The American Journal of Public Health, 105(7), 1302-1311.

Sommer, M., Schmitt, M., \& Clatworthy, D. (2017) A toolkit for integrating menstrual hygiene management (MHM) into humanitarian response. Columbia University and International Rescue Committee. Retrieved from https://www.mailman.columbia.edu/sites/default/files/pdf/mhmemergencies-toolkit-full.pdf

South, A. (2008). Ethnic Politics in Burma: States of Conflict. Abinggon, Oxon: Routledge.

Sphere, (2000). Humanitarian Charter and Minimum Standards in Disaster Response. Geneva, Switzerland: The Sphere Project.

Stravos, J., Godwin, L. N., \& Cooperrider, D. L. (2015). Appreciative inquiry: Organization development and the strengths revolution. In W. Rothwell, J. Stravos \& R. Sullivan (Eds.), Practicing Organization Development: Leading Transformation and Change (pp. 96-116). US: John Wiley \& Sons Inc

Stromquist, N. P. (2006). Gender, education and the possibility of transformative knowledge. Compare: A Journal of Comparative and International Education, 36(2), 145-161.

Sultana, F. (2007). Reflexivity, positionality and participatory ethics: Negotiating fieldwork dilemmas in international research. ACME: An International E-Journal for Critical Geographies, 6(3), 374385.

Tendero, J. (2019). 12 easy steps: Days for Girls. The Summer of Beautiful. Partners Journal 2019, 3237.

The Border Consortium. (2016). Annual Report. (January - December 2016). Retrieved from https://www.theborderconsortium.org/

The Border Consortium. (2019). Mae La. Retrieved from https://www.theborderconsortium.org/

The Gender Equality Network. (2015). Raising the Curtain: Cultural Norms, Social Practices and Gender Equality in Burma. Retrieved from http://www.burmalibrary.org

Tuck, E. in conversation with Michelle Fine (2007). Inner angles: A range of ethical responses to/with indigenous and decolonizing theories. In N. Denzin and M. Giardina (Eds.). Ethical Futures in Qualitative Research: Decolonizing the Politics of Knowledge, (pp. 145-168). Walnut Creek, CA: Left Coast Press.

UNESCO. (2014). Puberty Education \& Menstrual Hygiene Management. Paris, France: UNESCO. 
UNHCR. (2011) UNHCR's Commitments to Refugee Women. 12 December 2001. Retrieved from https://www.refworld.org/

UNHCR. (2019). Refugees in Thailand. Retrieved from https://www.unhcr.or.th/

United Nations (2015). Sustainable Development Goals. Goal 5: Achieve Gender Equality and Empower All Women and Girls. Retrieved from http://www.un.org/sustainabledevelopment/gender-equality/

. (2016). Day of the Girl Child: Girls' Progress = Goal's Progress: What Counts for Girls. Retrieved from http://www.un.org/youthenvoy/2016/10/girls-progress-goals-progress-counts-girls/

- (2019). Universal Declaration of Human Rights. Retrieved from https://www.un.org/en/universal-declaration-human-rights/

USIP. (2018). The Current Situation in Burma. Retrieved from https://www.usip.org/

Vanleeuwen, C., Torondel, B. (2018). Improving menstrual hygiene management in emergency contexts: literature review of current perspectives. International journal of women's health, 10, 169-186.

Walkling, K. \& Miedema, J. (2015). Eco Femme's Response to Sinu Joseph's Blog 'Why India Doesn't Need a Sanitary Pad Revolution. https://ecofemme.org/eco-femmes-response-to-sinu-josephsblog-why-india-doesnt-need-the-sanitary-napkin-revolution/

Walkup, J. T. (2012) Stigma. In Loue S., Sajatovic M. (eds) Encyclopedia of Immigrant Health. Springer, New York, NY.

Wanner, T., \& Wadham, B. (2015). Men and masculinities in international development: 'Menstreaming' gender and development? Development Policy Review, 33(1), 15-32.

Wilson, E. F., Reeve, J. M. K., Pitt, A. H., Sully, B. G., \& Julious, S. A. (2012). INSPIRES: Investigating a reusable sanitary pad intervention in a rural educational setting - evaluating the acceptability and short term effect of teaching Kenyan school girls to make reusable sanitary towels on absenteeism and other daily activities: a partial preference parallel group, cluster randomised control trial. (ScHARR Report Series 27). Scheffield, UK: University of Sheffield.

World Relief. (2018). Burma (Burma) Karen Cultural Profile. Retrieved from https://worldrelieffortworth.org/

Wu, B. (2014, October 30). Embedding research in local context: local knowledge, stakeholders' participation and fieldwork design. Retrieved from https://blogs.lse.ac.uk/ 
Yang, G. (2018). Karen Culture Presentation [Powerpoint slides]. Retrieved from https://www.ramseycounty.us

Zozimo, J. (2019). What if evaluation takes place seated around the table whilst enjoying a drink? A social practice view of evaluation of development education. Journal of International Development, 2019, 1-22. 


\title{
Appendix A: Participant Information Sheet ${ }^{6}$
}

\author{
HEC: 0000027545
}

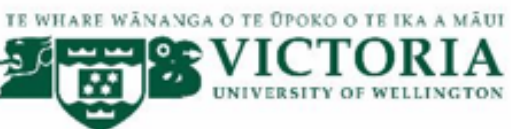

\section{Menstrual Hygiene Management Processes in Humanitarian Relief}

\section{INFORMATION SHEET FOR PARTICIPANTS -Partners Staff}

You are invited to take part in this research. Please read this information before deciding whether or not to take part. If you decide to participate, thank you. If you decide not to participate, thank you for considering this request.

\section{Who am I?}

My name is Tessa Bardsley and I am a Development Studies Masters student at Victoria University of Wellington, New Zealand. I am working with Partners Relief and Development on this research project.

\section{What is the aim of the project?}

This project aims to understand how women contribute to a programme of Menstrual Health Management (MHM) relief to create meaningful outcomes for displaced women on the Thai-Myanmar border. I am particularly interested in what you see as positive about your role in menstrual health relief processes. I'm trying to learn about ways that menstrual health relief can create more dignified outcomes for women. This research has been approved by the Victoria University of Wellington Human Ethics Committee [ResearchMaster application reference number \# 0000027545].

\begin{abstract}
How can you help?
You have been invited to participate because you have been identified as being part of the process of providing menstrual hygiene management relief. If you agree to take part, I will interview you in your workplace or in a location convenient to you. I will ask you questions about what you see as working well in your workplace and how you think processes could be improved. The interview will take 30-40 minutes. I will audio record the interview with your permission. You can choose to not answer any question I ask or stop the interview at any time, without giving a reason. You can withdraw from the study by contacting me at any time before September 15, 2019. If you withdraw, the information you have provided will be destroyed or returned to you.
\end{abstract}

\section{What will happen to the information you give?}

This research is confidential. This means that while the researchers named below will be aware of your identity, they will not use your name or describe you in a way that can identify you in any publications produced from the research. Before or after the interview, I will discuss with you about what information you are comfortable to have

\footnotetext{
${ }^{6}$ The information sheet, consent form, and question guide shared in the appendices are used as examples. In reality, I used a different version of each for each group (students, migrant community women, Partners staff and DfG Kit sewists).
} 
published. Only my supervisor and I will read the notes or transcript of the interview. The interview transcripts, summaries and any recordings will be stored in ways that do not directly identify you. They will be kept securely, and all research data will be destroyed in 3 years from September 2019. Deidentified results may be shared with other NGOs who provide Menstrual Hygiene Management humanitarian relief.

\section{What will the project produce?}

The information will go towards a report for Partners Relief and Development. It will also enable me to write a thesis as part of my Masters degree. It will help my supervisor and I to write a research article for publication and/or deliver presentations to interested organisations. We hope that the research project will help us to identify how menstrual health relief can create more dignified outcomes for women.

\section{If you accept this invitation, what are your rights as a research participant?}

You do not have to accept this invitation if you don't want to. If you do decide to participate, you have the right to:

- ask any questions about the study at any time;

- choose not to answer any question;

- ask for the recorder to be turned off at any time during the interview;

- choose a pretend name (to hide your own name);

- receive the transcript or summary of your interview and comment on it;

- $\quad$ withdraw from the study before September 15, 2019;

- receive a copy of my final research essay by emailing me.

If you have any questions or problems, who can you contact?

If you have any questions, either now or in the future, please feel free to contact either:

\section{Student:}

Name: Tessa Bardsley University email address:

bardslte@staff.vuw.ac.nz

\section{Supervisor:}

Name: Dr Sara Kindon

Role: Associate Professor

School: School of Geography,

Environment and Earth Sciences

Phone: 044636194

sara.kindon@vuw.ac.nz

\section{Human Ethics Committee information}

If you have any concerns about the ethical conduct of the research, you may contact the Victoria University HEC Convenor: Dr Judith Loveridge. Email hec@vuw.ac.nz or telephone +64-4-463 6028 . 


\section{Appendix B: Participant Consent Form}

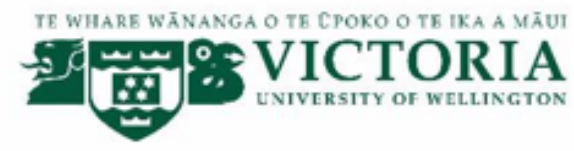

\section{Menstrual Hygiene Management Processes in Humanitarian Relief}

\section{CONSENT TO INTERVIEW - Reusable pad sewers}

I will work with a translator to make the information on this sheet accessible to participants

Researcher: Tessa Bardsley, School of Geography, Environment and Earth Sciences, Victoria University of Wellington.

- I understand the contents of the Information Sheet and the project has been explained to me. My questions have been answered to my satisfaction. I understand that I can ask further questions at any time.

- I agree to take part in an audio recorded interview.

I understand that:

- I may withdraw from this study at any point before June 4, 2020 and any information that I have provided will be returned to me or destroyed.

- The identifiable information, including this consent form, that I have provided will be destroyed by $31^{\text {st }}$ of December 2019. The interview transcripts, summaries and any recordings will be stored in ways that do not directly identify you. They will be kept securely, and all research data will be destroyed in 3 years from September 2019

- Any information I provide will be kept confidential to the researcher and the supervisor.

- I understand that the results will be used for a Masters Thesis and potentially for a research article, and that they may be shared with Partners Relief and Development and other NGOs providing Menstrual Health Management humanitarian relief.

- My name, nor any identifiable information, will not be used in any of these publications.

- $\quad$ I would like to review a translated summary of my interview $\quad$ Yes $\square$ No $\square$

- I would like to receive a copy of the final research essay and have Yes $\square$ No $\square$ added my email address below.

Signature of participant:

Name of participant:

Date:

Contact details: 


\section{Appendix C: Question Guide Example}

\section{DfG Kit Sewers Question Guide}

Intro:

- Where were you born?

- How old are you?

- How long have you been living in Mae Sot?

1. What role do you play in Partners' Days for Girls Programme?

- How long have you been working in the role?

- How did you get chosen for this role?

- What are your specific tasks or responsibilities?

2. Did you spend time in a refugee camp? If so:

- Could you tell me about the MHM methods you and women you knew used?

- Did you have access to MHM relief?

- How would DfG kits have impacted your life in the camp?

- Do you feel a connection with the women you sew the kits for? How so?

- Do you think there are other MHM methods that women would rather use? Why/why not?

3. Could you tell me what you know about Partners work in MHM relief?

- Where are the materials from?

- How are the kits created?

- What is it like to have conversations with others about your work with the DfG programme?

- When you talk about women's bodies and periods, is there awkwardness/curiosity/enthusiasm...?

4. What are the things you most value about your work?

- What has been a high point of your involvement in the programme so far?

- What skills/knowledge have you developed through working here?

- What do you hope to do with these skills in the future?

5. What changes have you seen for women in your community since the Partners MHM programme? Do you have any specific stories? Personal or from others you know?

6. What do you think could be improved in the programme to improve outcomes for:

- Women who sew the DfG kits?

-Women who receive the DfG kits?

5. If Partners had unlimited resources, how would you like to see Partners' MHM relief programme change?

- How do you think these methods could help women feel more dignity? Agency? 
Appendix D: Translator Agreement

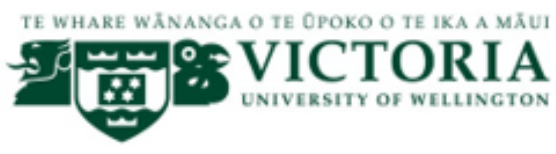

\section{Translation Confidentiality Agreement}

Project Title: Menstrual Hygiene Management Processes in Humanitarian Relief

Principal

Investigator: Tessa Bardsley

$\mathrm{I}$, , agree to ensure that the interviews

I translate will remain confidential to Tessa Bardsley and myself. I agree that I will not discuss any aspect of the interviews with anyone except Tessa Bardsley.

I agree that I will remain impartial and unbiased in my translation of all interviews.

Name:

Signature:

Date: 